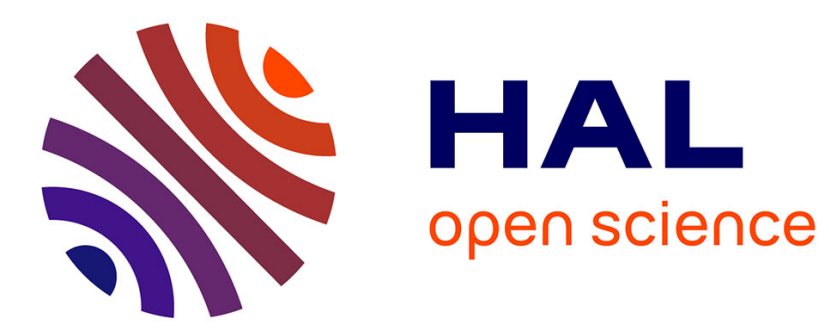

\title{
Accurate simulation of the noise generated by a hot supersonic jet including turbulence tripping and nonlinear acoustic propagation
}

Adrien Langenais, Vuillot François, Julien Troyes, Christophe Bailly

\section{To cite this version:}

Adrien Langenais, Vuillot François, Julien Troyes, Christophe Bailly. Accurate simulation of the noise generated by a hot supersonic jet including turbulence tripping and nonlinear acoustic propagation. Physics of Fluids, 2019, 31 (31), pp.016105-1 - 016105-31. 10.1063/1.5050905 . hal-02050769

\author{
HAL Id: hal-02050769 \\ https://hal.science/hal-02050769
}

Submitted on 1 Mar 2019

HAL is a multi-disciplinary open access archive for the deposit and dissemination of scientific research documents, whether they are published or not. The documents may come from teaching and research institutions in France or abroad, or from public or private research centers.
L'archive ouverte pluridisciplinaire HAL, est destinée au dépôt et à la diffusion de documents scientifiques de niveau recherche, publiés ou non, émanant des établissements d'enseignement et de recherche français ou étrangers, des laboratoires publics ou privés. 


\title{
Accurate simulation of the noise generated by a hot supersonic jet including turbulence tripping and nonlinear acoustic propagation
}

\author{
A. Langenais, ${ }^{1, \text { a) }}$ F. Vuillot, ${ }^{1, b)}$ J. Troyes, ${ }^{2, \text { c) }}$ and C. Bailly ${ }^{3, \mathrm{~d})}$ \\ 1) DMPE, ONERA, University of Paris-Saclay, Palaiseau, France \\ ${ }^{2)}$ DMPE, ONERA, University of Toulouse, Toulouse, France \\ ${ }^{3)}$ LMFA, École Centrale de Lyon, University of Lyon, Lyon, France
}

(Dated: Submitted: 3 August 2018 • Accepted: 8 December 2018 • Published online: 30 January 2019)

\begin{abstract}
A procedure to accurately simulate a free hot supersonic jet and its associated noise, which uses simultaneously a turbulence tripping method and a two-way coupling between a flow solver and a nonlinear acoustic solver, is proposed in this study. A Mach 3.1 overexpanded hot jet is computed via a large-eddy simulation by solving the filtered Navier-Stokes equations with a finite volume method on unstructured grids. The resulting noise is propagated in the far field by solving the full Euler equations with a high-order discontinuous Galerkin method on unstructured grids. The full convergent-divergent nozzle is explicitly included in the computational domain thanks to the unstructured flow solver. Both a refined grid and a geometrical boundary layer tripping in the convergent are used to get highly disturbed turbulent conditions at the nozzle lips. The flow field appears to agree with the expected turbulence behavior and the available experimental data. The jet development shows significant improvement compared to similar past simulations. The far field acoustic levels are finely recovered at most of observation angles. An analysis of the acoustic near and far fields is then performed. The studied conditions lead to strong shock-associated noise and Mach wave emission. The spatio-frequency and azimuthal content of the acoustic field is described in order to identify the main noise properties. A particular noise component, different from screech tones and radiating upstream like Mach waves, is highlighted. Nonlinear propagation effects are finally quantified through specific metrics. They are found significant in both the near and the far fields which justifies the use of a nonlinear acoustic solver.
\end{abstract}

\section{INTRODUCTION}

Understanding and controlling the noise radiated by rocket engine jets at lift-off is a major issue for space applications. Imperfectly expanded hot supersonic jets are generated and deflected in a flame trench at lift-off, generating a harsh acoustic field which induces severe loads on the launcher, the payload and the surrounding structures. ${ }^{1}$ Since the founding work of Lighthill, supersonic jet noise has been carefully investigated, as extensively reviewed by $\mathrm{Tam}^{2}$ or Bailly and Fujii. ${ }^{3}$ Experimental studies have first been conducted to identify noise sources as reported by Tam et al. ${ }^{4}$ for instance. Based on measured data, three main components have been highlighted: the turbulent mixing noise, the broadband shock-associated noise and the Mach wave radiation. Numerous authors have contributed by studying specific mechanisms including nonlinearities and crackle, ${ }^{5-8}$ screech and feedback effects, ${ }^{9-11}$ interaction with obstacles ${ }^{10,12}$ or with noise reduction devices. ${ }^{13}$ Available experimental studies regarding imperfectly expanded hot supersonic jets at Mach number $M>3^{14}$ are however limited.

\footnotetext{
a)Electronic mail: adrien.langenais@onera.fr; DMPE, ONERA, University of Paris-Saclay, Palaiseau, France

b)Electronic mail: francois.vuillot@onera.fr

c) Electronic mail: julien.troyes@onera.fr

d) Electronic mail: christophe.bailly@ec-lyon.fr
}

Semi-empirical noise source and propagation models have been developed in paralle ${ }^{15}$ but their ability to predict realistic installation effects remains poor. On the contrary, numerical simulations of the Navier-Stokes equations give access to detailed flow information on increasingly complex configurations.

The large-eddy simulation (LES) of a Mach 3.0 cold jet by Nonomura et al. ${ }^{16}$ with focuses on overexpansion effects, and the simulation of Mach 1.3-1.5 planar and round cold jets by Gojon et al. ${ }^{17}$ and Bogey et al. ${ }^{18}$ with emphasis on feedback mechanisms, can be mentioned as advanced numerical works. Imperfectly expanded hot supersonic jet computations have also been performed over the past decade. De Cacqueray et al. ${ }^{19}$ have simulated a Mach 3.3 heated overexpanded free jet, investigating noise generation mechanisms. Brès et al. ${ }^{20}$ have simulated a Mach 1.5 heated overexpanded free jet on various meshes, underlining the importance of addressing the boundary layer inside the nozzle and the turbulent state.

The jet initial conditions at the nozzle exit, especially the turbulence level close to the lips, have been found to be critical to perform high fidelity computations. ${ }^{21-24} \mathrm{It}$ has been observed experimentally that increasing the initial turbulence level expands the shear layer thickness ${ }^{25}$ and its spread rate. ${ }^{26}$ Long et al. ${ }^{25}$ have also found that increasing the initial turbulence level leads to a reduction of the screech tones and of the shock-associated noise, suggesting a major influence of turbulence properties on the interaction mechanisms between turbulence and shocks. Fontaine et al..$^{27}$ have demonstrated 
that a thicker initial shear layer induces a significant noise reduction. Similar trends have been obtained numerically. ${ }^{28}$ Liu et al..$^{22}$ have performed simulation of underexpanded supersonic jets, explicitly including the nozzle geometry, and have also shown that the shear layer thickness increased when the initial turbulence level is raised from $1.2 \%$ to $7 \%$. However, a minor impact on noise levels has been observed. On the contrary, the work by Bogey et al. ${ }^{21}$ on a subsonic jet has demonstrated an important reduction of the noise overestimation when increasing the initial turbulence level from $0 \%$ to $12 \%$. Main effects have appeared when moving from $0 \%$ to $3 \%$ and have been attributed to a weaker vortex pairing in the shear layer. Consequently, many computations now use a forcing strategy to mimic the incoming turbulent boundary layer. It consists in either a flow seeding with synthetic disturbances as performed by Bogey et al. ${ }^{28}$ Gloor et al. ${ }^{29}$ and Brès et al. ${ }^{24}$ for instance, or a geometrical tripping upstream of the nozzle exit as described by Liu et al., ${ }^{22}$ Lorteau et al. ${ }^{23}$ or Brès et al. ${ }^{20}$ Mesh refinement, particularly in the azimuthal direction, is also admitted to be a key feature to accurately simulate supersonic jets and the generated noise by LES. ${ }^{21,23,30}$

The constantly increasing available computing resources allow even more accurate LES or direct flow computation. Including the whole acoustic field in such approaches however remains out of reach for realistic applications. The acoustic far field can be calculated by an integral acoustic formulation applied to a control surface ${ }^{31}$ such as the Kirchhoff or the Ffowcs Williams \& Hawkings (FWH) integral methods. Nonlinear propagation effects induced by high sound pressure levels (SPL) in space applications, such as waves distortion, and installation effects linked to the launch pad geometry, are not readily taken into account by these approaches.

Coupling methods between flow and acoustic solvers have been evaluated and applied to jet noise. The Navier-Stokes equations (NS) are usually solved by LES and weakly coupled to the linearized Euler equations (LEE) ${ }^{32,33}$ that is with no feedback from the acoustic region. More recently, de Cacqueray et al. ${ }^{19,34}$ have simulated the noise emitted from a Mach 3.3 jet with a one-way Navier-Stokes-Euler coupling using a highorder structured solver. An analysis of far field noise components and a quantification of nonlinear propagation effects have been conducted. Harris et al. ${ }^{35,36}$ have overlapped an unstructured LES and a discontinuous Galerkin acoustic solver to run a 2D simulation of the Space Launch System acoustic environment. Labbé et al. ${ }^{37}$ have performed a one-way NavierStokes-Euler coupling for subsonic jet noise prediction. Results have been compared to those obtained from the Ffowcs Williams \& Hawkings integral method and have agreed in linear regime as expected. Tsutsumi et al. ${ }^{38}$ have applied a one-way Navier-Stokes-Euler coupling to a reduced scale flame trench, showing the ability of the method to treat realistic launch pad configurations. Space applications may imply complex secondary flows and acoustic feedback on the primary flow which can be taken into account only with a two-way method. Two-way coupling between fully 3D unstructured NavierStokes and Euler solvers has been recently developed and validated for space applications. ${ }^{39}$

Finally, the presence of acoustic nonlinearities in the near field is admitted ${ }^{6,40}$ but the need of a nonlinear acoustic solver to compute the far field propagation is still debated. The dimensionless Goldberg number $\Gamma$ can be introduced ${ }^{7}$ as an indicator to distinguish regions where cumulative acoustic nonlinearities are dominant compared to the atmospheric damping. The noise levels observed in space applications suggest that significant cumulative nonlinearities occur in the far field, resulting in waveform steepening, shock coalescence and amplitude loss compared to linear propagation. ${ }^{41,42}$ Specific metrics have been proposed and assessed to quantify the nonlinear propagation, especially the pressure derivative skewness, the pressure derivative kurtosis and the wave steepening factor. ${ }^{7,8,43}$ De Cacqueray et al. ${ }^{19}$ have compared the far field propagation of the noise generated by a Mach 3.3 jet computed with the linearized and the full Euler equations. The LEE simulation have resulted in a $5 \mathrm{~dB}$ noise overestimation at $120 D$ in the peak direction. Similar trends have been observed by comparing a Ffowcs Williams \& Hawkings and a full Euler computations. ${ }^{44}$ The FWH method overestimates the far field noise by about $2 \mathrm{~dB}$ at $40 \mathrm{D}$ in the peak direction. These evidences clearly indicate that nonlinear propagation effects cannot be ignored in the far field.

In the present work, a free hot supersonic jet at a Mach number 3.1 is simulated using simultaneously a turbulence tripping method and a two-way coupling between unstructured flow and acoustic solvers. The solvers are respectively based on the Navier-Stokes equations written for LES and solved by a finite volume method and the full Euler equations solved by an high-order discontinuous Galerkin method. The study is organized in three main parts. First, the simulation methodology is described in part II. The main jet properties, the comparison database and the numerical set-up are detailed in section II A. The numerical tools are presented in section II B. Then, major grid effects are discussed in section II C. A geometrical turbulence tripping method is implemented in section IID. The resulting flow inside the nozzle is also described. Secondly, the free jet flow computation and the resulting noise are carefully validated in part III by comparing results to available experimental and numerical data. The sections III A and III B are respectively dedicated to aerodynamic and acoustic validation. Thirdly, main noise sources and acoustic propagation phenomena are analyzed in part IV. The noise sources are discussed $a$ priori and linked to the computed acoustic field in section IV A. Properties of the velocity and pressure near fields and of the pressure far field are characterized respectively in sections IV B and IV C. Finally, the nonlinear acoustic propagation effects are highlighted and quantified in section IV D. Concluding remarks are given in part V. 


\section{SIMULATION METHODOLOGY}

\section{A. Jet case set-up and database}

\section{Physical parameters and notations}

An overexpanded hot jet with an exit Mach number $M_{j}=u_{j} / c_{j}=3.1$ and a Reynolds number $R e_{D}=$ $u_{j} D / \nu_{j}=3 \times 10^{5}$ is considered, where the subscript $j$ refers to the jet conditions on the centerline in the nozzle exit plane, $u$ the velocity, $c$ the speed of sound, $D$ the diameter and $\nu$ the kinematic viscosity. The corresponding ideally-expanded Mach number is $M_{d}=2.8$. The jet fluid is composed of an equivalent propellant gas resulting from an hydrogen-air combustion. The specific heat ratio is $\gamma=1.3$. The generating conditions are $p_{i}=30 \times 10^{5} \mathrm{~Pa}$ for the total pressure and $T_{i}=1900 \mathrm{~K}$ for the total temperature. The jet is exhausted from a convergentdivergent nozzle with an exit diameter $D=60 \mathrm{~mm}$, at conditions $T_{j} / T_{\infty}=2.6$ and $p_{j} / p_{\infty}=0.6$. Ambient medium is air at $\gamma=1.4, T=T_{\infty}=293.15 \mathrm{~K}$, $p=p_{\infty}=1 \times 10^{5} \mathrm{~Pa}$. The main flow parameters are recalled in Table $\mathrm{I}$. The generated noise reaches very high acoustic levels typically greater than $130 \mathrm{~dB}$ out to $40 \mathrm{D}$ from the sources. In the following, all positions and length quantities referred by the superscript * are made dimensionless by the jet exit diameter $D_{j}$. All dB scales are calculated with a reference value of $2 \times 10^{-5}$ SI.

\section{Experimental and numerical database}

The present jet parameters correspond to those of an experimental study conducted at the same scale in the MARTEL semi-anechoic facility. The hydrogen-air combustion provides generating conditions in range $p_{i}=$ $25-30 \times 10^{5} \mathrm{~Pa}, T_{i}=500-2100 \mathrm{~K}$ and a maximum overexpanded exit velocity of $1800 \mathrm{~m} / \mathrm{s}$. The test bench aims at investigating supersonic, free or impinging jet noise at launcher lift-off conditions from a reduced scale motor (about 1/40 based on the Ariane 5 solid propellant boosters). The experimental set-up for the considered case is depicted in Figure 1a. Two-component laser Doppler velocimetry (LDV) and far field acoustic measurements have been carried out. Measurements include axial and radial profiles of mean axial $\bar{u}_{x}$ and radial $\bar{u}_{r}$ velocities, root mean square (RMS) fluctuating axial $u_{x}^{\prime}$ and radial $u_{r}^{\prime}$ velocities, axial velocity skewness $S\left(u_{x}\right)$ and kurtosis $K\left(u_{x}\right)$, along the jet axis and along four radii positioned at $x^{*}=x / D_{j}=1,3,3.67$ and 6 . A microphone arc of ra-

TABLE I: studied supersonic jet operating conditions.

\begin{tabular}{ccccccc}
\hline \hline$M_{j}$ & $M_{d}$ & $p_{j} / p_{\infty}$ & $T_{i} / T_{\infty}$ & $R e_{D} \times 10^{-6}$ & $D$ & $D_{j}$ \\
3.1 & 2.8 & 0.6 & 6.5 & 0.3 & $60 \mathrm{~mm}$ & $0.97 D$ \\
\hline \hline
\end{tabular}

dius $41.6 D$, centered on the nozzle exit, provides far field acoustic measurements. The wedges aim at isolating microphones from reflected waves by the ground and the small central region without wedges is not expected to contribute to recorded signals (Figure 1a not at scale). Microphones are spaced every $\Delta \theta=10^{\circ}$ and their frequency bandwidth is $50 \mathrm{kHz}$.

LES simulations of the same free jet have first been performed by Troyes et al. ${ }^{45}$ The acoustic far field was computed with the Ffowcs Williams \& Hawkings method. ${ }^{31}$ More recently, Langenais et $a l^{44}$ have adapted the case by using an identical near field mesh and computing the far field with the full Euler equations. Resulting aerodynamic and acoustic fields provide a valuable numerical database which will be referred as Langenais $2017^{44}$ in the following.

\section{Computational set-up}

In the present numerical investigation, the jet and the acoustic near field are simulated by solving the NavierStokes equations by LES in the region hereafter denoted $\mathrm{Z} 1$ and the acoustic far field is computed solving the full Euler equations in the region Z2 as illustrated in Figure 1b. The two solvers exchange data at a conical coupling interface S1. The origin of the coordinate system is located at the nozzle exit plane on the jet axis. The cylindrical coordinates system $(x, r, \Theta)$ centered on the jet axis is preferred for analysis convenience. The full convergentdivergent nozzle geometry is explicitly included in the domain. Qualitatively based on a previous flow solution, ${ }^{44}$ the coupling interface radius is chosen as $r=2.7 x+3.8$ in order to follow the jet expansion. The ground is not included in the domain which extends $33.5 \mathrm{D}$ upstream, $81.6 D$ downstream and $59.4 D$ laterally. In addition to a surrounding buffer zone depicted in Figure 1c where the grid is stretched, a characteristic-based non-reflective condition $^{46}$ is set at the domain boundary in the zone Z2 and a standard outflow condition with an imposed pressure $p=p_{\infty}$ is set downstream in the zone Z1. Finally, far field recording points are placed at the experimental microphone locations in the zone $\mathrm{Z} 2$, as well as at the LDV measurement locations in the zone $\mathrm{Z} 1$.

The main computation has been performed on 1736 Broadwell processors of the ONERA's parallel scalar cluster SATOR, including 1512 cores allocated to the Navier-Stokes solver and 224 cores allocated to the Euler solver. A total physical time of $1180 \mathrm{D} / \mathrm{u}_{j}$ has been simulated, which provides a fully established state duration of $650 \mathrm{D} / u_{j}=130 \mathrm{D} / \mathrm{c}_{\infty}$ as exploitable time. It is comparable to similar computations in the literature ${ }^{19,20}$ but can be limited in terms of temporal convergence for low frequencies. The minimum accessible frequency is $S t_{\min }=1.5 \times 10^{-3}$ but in practice, a relevant low frequency bound considering at least five periods is $S t_{\min }=$ $8 \times 10^{-3}$. The total computational cost is $4.5 \times 10^{6} \mathrm{CPUh}$. 


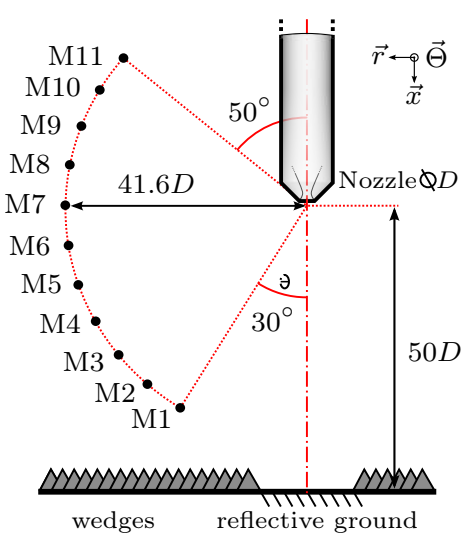

(a)

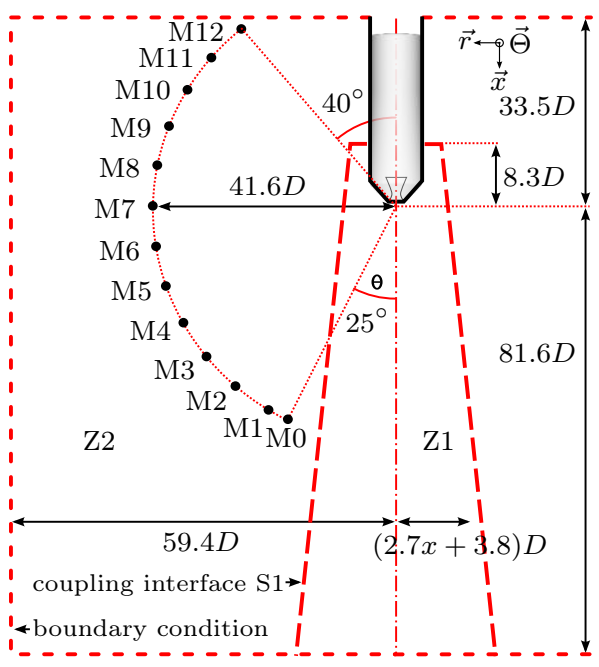

(b)

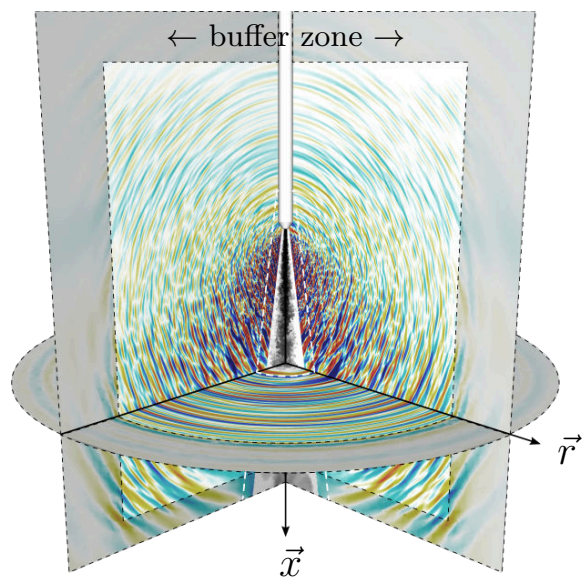

(c)

FIG. 1: dimensions of (1a) the experimental set-up at MARTEL facility and (1b) the present computational set-up; (1c) visualization of the 3D numerical domain.

\section{B. Numerical methods and settings}

\section{Flow computation}

The ONERA multi-physics code CEDRE which operates on general unstructured elements ${ }^{47}$ is used in the zone Z1. The filtered compressible Navier-Stokes equations are solved with a Smagorinsky subgrid scale model with a constant value $C_{s}=0.1$. No wall model is used but the van Driest damping function is implemented. The specific heat is defined by a $7^{\text {th }}$ order polynomial for air and is set constant for the propellant gas. Species viscosity is calculated according to the Sutherland law. The time integration is performed with an implicit $2^{\text {nd }}$ order Runge-Kutta scheme associated with a GMRES type linear system solver. The flux calculation is carried out with a $2^{\text {nd }}$ order MUSCL method and an HLLC flux scheme. The required spatial discretization is characterized in terms of point per wavelength (PPW). This criterion is linked to the computation cut-off Strouhal number as

$$
S t_{c}=\frac{f_{c} D_{j}}{u_{j}}=\frac{c_{\infty} D_{j}}{\lambda_{c} u_{j}}=\frac{c_{\infty} D_{j}}{(\mathrm{PPW} \times K \times \Delta) u_{j}}
$$

where $f_{c}$ is the cut-off frequency, $\lambda_{c}$ the cut-off wavelength, $\Delta$ the grid characteristic size and $K$ a correction constant. Based on preliminary computations and previous studies, the $K$ parameter is set to 1.2 to match the prediction with the observed cut-off frequency. The setting of $\Delta$ is discussed in section II C 2 dealing with the grid parameters. A resolution of PPW $=20$ has been found to be required for a proper acoustic propagation in the near field with the CEDRE code. ${ }^{45}$ The associated numerical damping reaches its maximum value of $0.5 \mathrm{~dB} / \lambda_{c}$ at the cut-off.

\section{Acoustic computation}

The ONERA unstructured computational aeroacoustics (CAA) code SPACE ${ }^{48,49}$ is used in the zone Z2. The full Euler equations are solved through a nodal discontinuous Galerkin method using high-order polynomial elements without order limit. Thermodynamic properties of the propagation medium (air) are set constant with $\gamma=1.4$. The time integration is performed with an explicit $2^{\text {nd }}$ order Runge-Kutta scheme and high spatial orders up to the $4^{\text {th }}$ order are used. It has been established that high-order methods for aeroacoustic problems are beneficial in terms of cost/accuracy ratio. ${ }^{50}$ According to Delorme et al. ${ }^{48}$ a PPW $=14$ discretization is required at the $2^{\text {nd }}$ spatial order and only $\mathrm{PPW}=3$ at the $4^{t h}$ order for an ideal case of wave propagation. The cut-off Strouhal number is linked to PPW via equation (1) and $K$ is set to 1.2 as previously. An higher resolution of PPW $=5$ at the $4^{\text {th }}$ order is chosen here. The resulting numerical dissipation is of the order of the atmospheric viscous damping, i.e. $\sim 10^{-3} \mathrm{~dB} / \lambda$ over the resolved spectrum. ${ }^{51}$ The discontinuous Galerkin method implemented in SPACE also allows the user to locally adapt the spatial orders. This method is called order mapping or $p$-adaptation and greatly improves the usage convenience. A description of the non-reflective boundary conditions and the shock-capturing scheme can be found in Langenais et al. ${ }^{39}$ 


\section{Two-way coupling}

The flow and the acoustic solvers are linked through a two-way surfacic coupling. Cell location, data exchange and space interpolation are carried out with the CWIPI coupling library ${ }^{47,52}$ via MPI communications. No mesh overlapping is used at the coupling interface. The same space discretization is considered at the interface in both solvers, i.e. conformal tetrahedral meshes. The coupling algorithm consists in locating the boundary cells on each side of the interface, then calculating and exchanging the values required by the other code at cell centers for $\mathrm{CE}$ DRE and nodes for SPACE, at every time step. Note that the use of conformal grids and the exchange at every time step increases the coupling robustness and allows to minimize the signal degradation which could be induced by spatial or temporal aliasing. However, it implies additional constraints such as a same time step in both solvers. The values of the conservative variables sent to SPACE are calculated from CEDRE primitive values $(p$, $T, \boldsymbol{u})$ with SPACE thermodynamic laws. Multi-species LES simulations can therefore be carried out without pressure, temperature or velocity discontinuities across the interface. At the end of a coupling cycle, exchanged values are used by each solver to compute the numerical flux at the interface. An extended description of the coupling features and a set of appropriated validation test cases can be found in Langenais et al. ${ }^{39}$

Since the flow and the acoustic computations are respectively second and fourth order accurate, the coupling interface can be seen as an accuracy discontinuity which could generates numerical instabilities. This issue is treated by locally adapting the element order close to the interface on the SPACE side. The small size cells induced by the conformal meshes at the interface are set to the second order. Moving away from the interface, the cell size increases and the element order quickly rises to the third order and finally, to the fourth order. This method provides a smooth transition in terms of numerical accuracy without reducing the global computation order since the flow solution remains second order accurate.

The full unstructured coupling methodology is able to deal with complex geometries and acoustic feedback on the flow. In the present free jet case, the benefits of these features mainly lie in the ability to easily mesh the nozzle and to take into account the induced secondary flow given that no acoustic feedback is expected.

\section{Grid effects}

\section{Past efforts}

A common supersonic nozzle used in a series of MARTEL test campaigns (see §II A 2) has formed the basis of several sets of LES computations at ONERA aiming at predicting the acoustic far field. Troyes et al. have simulated jet cases corresponding to experiments con- ducted at the MARTEL test bench. ${ }^{45}$ Major grid effects have been investigated. A sufficiently refined unstructured mesh has been found to be required on the jet axis to accurately capture shock cell locations. The azimuthal resolution at the nozzle lips has been found to significantly influence the early shear layer development. More recently, the far field mesh of a same free jet case ${ }^{45}$ has been adapted by Langenais et $a .^{44}$ to perform a Navier-Stokes-Euler coupled computation following the procedure described in section II B 3. Since the near field mesh and flow solution were unchanged, it has allowed to specifically study the impact of the nonlinearities on the acoustic far field. This work has also concluded that further mesh refinement was needed to better resolve the jet flow solution, especially the early jet development.

\section{Present improvement}

The present work involves a refined mesh described in the current section and a geometrical turbulence tripping method consisting in a small step added at the nozzle wall as discussed in next section II D.

The grid is unstructured and mainly composed of tetrahedra. The refinement effort is focused on the nozzle wall, the lips, the shear layer and the first two shock cell centerline regions. The motor body and the nozzle wall are meshed with a dozen of hexahedron layers. The cell sizes are reported in Table II at key locations in terms of axial, azimuthal and radial characteristic lengths for the hexahedra and characteristic edge for the tetrahedra. The corresponding equivalent cell diameter $d_{\text {cell }}$ is chosen as characteristic grid size $\Delta$ and is given by

$$
d_{\text {cell }}=\Delta=\frac{6 V}{A} \simeq \frac{\sqrt{6}}{6} e
$$

where $A$ is the cell faces total area, $V$ the cell volume and $e$ the tetrahedron edge. Note that $d_{c e l l}$ is strictly equal to $(\sqrt{6} / 6) e$ only in case of a regular tetrahedron. The hexahedron layers in the nozzle are not refined enough to fully resolve the boundary layer $\left(y^{+} \simeq 12\right.$ at the nozzle throat, $y^{+} \simeq 10$ at the separation point) but enough to convect the forced velocity fluctuations as part of the turbulence tripping method discussed in next section. The hexahedra at the nozzle lips provide an azimuthal resolution of about $2 \pi / \Delta \theta \simeq 1300$ cells which is nearly three times the resolution of the previous grid. ${ }^{44}$ The axial variation of $d_{\text {cell }}$ inside the nozzle boundary layer and along the lipline in the shear layer is plotted in Figure 2. The drop at $x=0$ can be explained by the transition between hexahedral and tetrahedral elements. Downstream, the tetrahedron stretching is nearly linear and the stretching rate tends to $\mathrm{d}\left(d_{\text {cell }}\right) / \mathrm{d} x=0.001$ which is two order of magnitude lower than a representative shear layer spread rate for such a supersonic jet. ${ }^{26}$ It should enable a proper development of the shear layer and a good resolution of the turbulent spectra. The cell size indicated for the Navier-Stokes acoustic field in Table II is maintained up 
TABLE II: cell characteristic size at key locations.

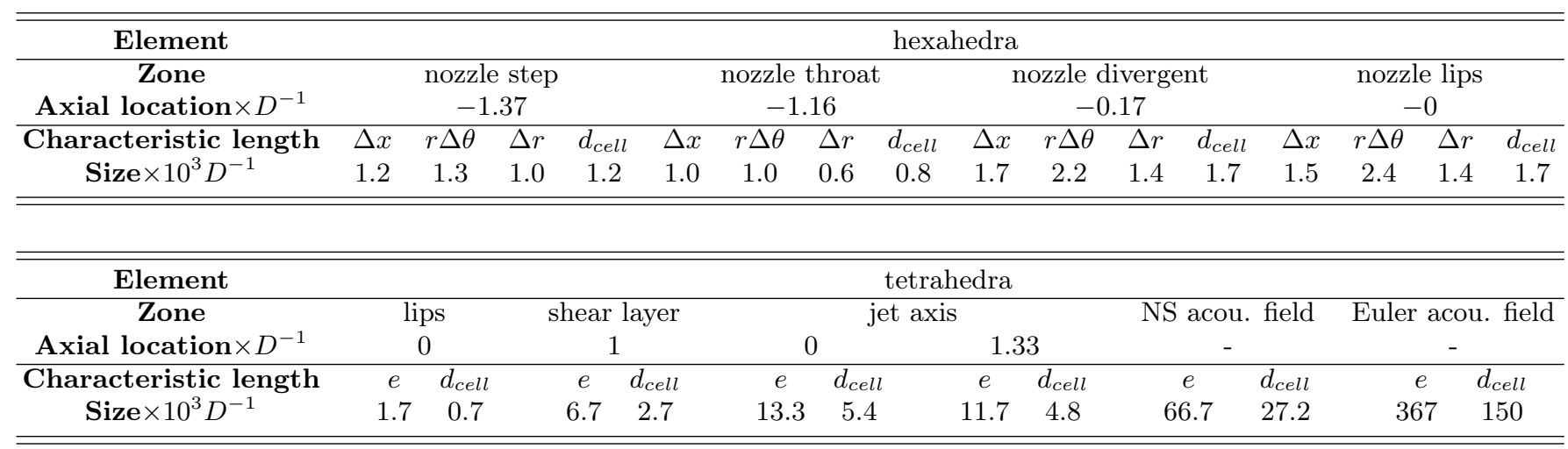

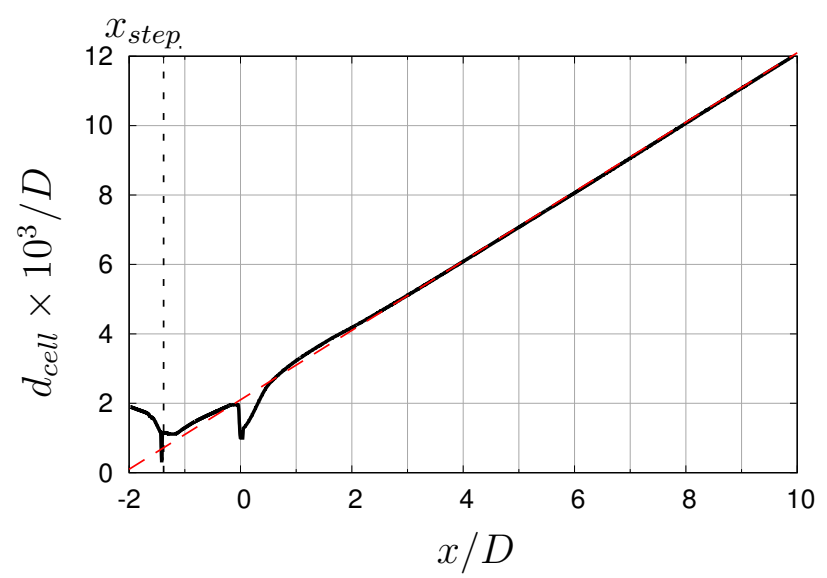

FIG. 2: characteristic cell diameter $d_{c e l l}$ in the nozzle wall boundary layer and the shear layer. - mesh; ---: asymptotic stretching rate $\mathrm{d}\left(d_{\text {cell }}\right) / \mathrm{d} x=0.001$.

to $x=20 D$. Downstream, the mesh is slowly coarsened by doubling the cell size in the range $20 D<x<42 D$. The cell size indicated for the Euler acoustic field is maintained up to $x=-28 D$ upstream, $x=42 D$ downstream and $r=42 D$ laterally. According to equation (1) and $d_{\text {cell }}$ values reported in Table II, the cut-off frequencies of the Navier-Stokes acoustic field $(x \leq 20 D)$ is $S t_{c}=0.30$ considering PPW $=20$ (see §II B 1) which is slightly overrated for the acoustics because of the aerodynamics. The cut-off frequencies of the Euler acoustic field is $S t_{c}=0.22$ considering $\mathrm{PPW}=5$ (see $\S \mathrm{II} \mathrm{B} 2$ ) while the cut-off frequency of the previous computation was $S t_{c}=0.15 .{ }^{44}$ The entire mesh is composed of $290 \times 10^{6}$ cells including $235 \times 10^{6}$ cells in Z1 and $55 \times 10^{6}$ cells in Z2.

\section{Turbulence tripping}

\section{Tripping strategy}

The previous computation of the present free jet case ${ }^{44}$ has shown a lack of turbulent fluctuations in the shear layer at $x^{*}=1$ in regard to the experimental levels. It has resulted in a too rapid laminar-turbulent transition, a too strong vortex pairing and a significant noise overestimation at mid frequencies. As examined previously in the introduction, numerical simulations at high Reynolds number require a forcing strategy to trigger the transition from a laminar to a turbulent state. ${ }^{28}$ The chosen approach here consists in a geometrical tripping as implemented in other existing studies. Liu et al. ${ }^{53}$ have added a sawtooth surface roughness in the convergent of a supersonic nozzle. The roughness height is $1 \%$ of the nozzle diameter and enables a $5 \%$ turbulence level increase at the nozzle lips. Lorteau et al. ${ }^{23}$ have used a rectangular step in a converging subsonic nozzle. The step height is $0.5 \%$ of the nozzle diameter and the shape is axisymmetric to avoid spurious azimuthal mode excitation.

In the present tripping strategy, an axisymmetric step is added at the nozzle wall at an axial location $x_{\text {step }}$. A parametric study is carried out to optimize the tripping benefit as summarized in section IID 2. The resulting wall flow inside the nozzle is described in section IID 3 in the case of the full computation.

\section{Parametric study}

The parametric study is conducted by varying the step main parameters, i.e. the axial location $x_{\text {step }}$, the height $h$ and the top edge slope. Five step configurations have been investigated, named steps A, B, C, D and without step, on a reduced computational domain. The detailed configurations and procedure are provided in appendix A. The step $\mathrm{C}$ located in the convergent, with parameters $x_{\text {step }}=-1.38 D, h=0.01 D$ and the edge slope parallel to the axis $\vec{x}$, is implemented in the full computation. It provides a highly disturbed flow close to the nozzle lips with fluctuation levels $u^{\prime} / u_{j}>3 \%$ while the configuration without step leads to $u^{\prime} / u_{j}<0.5 \%$ on a comparable grid. The full mesh in the zone Z1 finally includes $235 \times 10^{6}$ cells which adds $17.7 \times 10^{6}$ cells compared to the base without trip. 


\section{Nozzle wall flow}

Figures 3 and 4 show snapshots of Q-criterion (3a) and vorticity ( $3 \mathrm{~b}$ and 4 ) inside the nozzle. The effect of the step $\mathrm{C}$ is clearly visible. A separated flow and a recirculation zone of length $\simeq 4 h$ are generated above and immediately downstream of the step as it can be seen on the mean velocity profiles in Figure 5a. Vortical structures and velocity fluctuations are generated and convected downstream within the boundary layer. Axial velocity fluctuations reach important root mean square levels up to about $15 \%$ of the mean velocity on the axis as shown in Figure 5b. This figure also illustrates the mesh discretization near the wall. The power spectral density (PSD) of the velocity fluctuations immediately downstream of the step (not plotted) appears to be broadband centered on $S t_{\text {step }}=f h / u_{0} \sim 0.01$, where $u_{0}$ is the mean velocity outside the boundary layer above the step. The generated structures are then strongly stretched at the throat and in the divergent which can be associated with a relaminarization phenomenon as expected for such highly accelerated compressible flow. ${ }^{54}$ A similar stretching has been observed by Liu et al. ${ }^{53}$ for their supersonic jet simulation also using a geometrical turbulence tripping.

The main boundary layer properties are depicted in Figure 6. The maximum of axial velocity fluctuation is given in Figure 6a and its radial location in Figure 6b. Initially, the fluctuation levels are negligible. Then, the step induces a strong increase due to the recirculating flow, followed by a quick fall in the throat area. A similar trend can be qualitatively observed on the vorticity fields in Figure 4. The vorticity intensity greatly increases downstream of the step (Figure 4b). Then, the flow acceleration in the nozzle induces relaminarization, tends to thin the boundary layer and decreases the vorticity intensity. It explains the fluctuation drop at the throat. This is corroborated by the maximum location which moves closer to the wall at the throat. Despite relaminarization, large vortical structures are still noticeable at the throat (Figure 4c). Significant vorticity (Figure $4 \mathrm{~d}$ ) and fluctuation levels $(\geq 2 \%)$ are saved downstream in the divergent up to the nozzle exit (Figure 4e) which should lead to a proper early shear layer destabilization. The additional fluctuation raise before the nozzle exit is attributed to a small flow separation occurring at the lips. Such a separation is not expected to occur for the present jet parameters (see $\S I I$ A 1 ) according to the Morisette and Goldberg's criterion, ${ }^{55}$ i.e. $p_{j} / p_{\infty}<0.3$ at $M=3.1$. However, a separation has also been obtained in the simulation by Liu et al. ${ }^{53}$ where the criterion, i.e. $p_{j} / p_{\infty}<0.65$ at $M=1.5$, is not met either. It can be explained as a side effect of the wall mesh refinement which allows to capture the subsonic sub-boundary layer and favor the flow separation in case of overexpanded exit conditions. The separation goes up to $0.17 D$ upstream of the nozzle exit and is about $0.015 D$ wide, as illustrated in Figure $7 \mathrm{a}$, which implies to consider that $D_{j}$ is only equal to $97 \%$ of the actual nozzle exit diameter $D$. This correction is systematically applied (superscript ${ }^{*}$ ), except for domain dimensions. The separation is strictly steady and the raise of $u_{x}^{\prime}$ for $x>-0.17 D$ can be more likely attributed to a standard free shear layer behavior since the flow is not guided anymore by the nozzle wall in this region. Given the local pressure inside the jet, the ambient air is sucked in, inducing a counter flow as noticeable on the profile in Figure $7 \mathrm{c}$.

The boundary layer dimensionless thickness $\delta_{b l}^{*}$, displacement thickness $\delta^{*}$, momentum thickness $\theta^{*}$ and shape factor $H$ are given in Figure 6. This quantities are calculated for the present compressible flow according to

$$
\begin{aligned}
& \delta_{b l}^{*}\left(x^{*}\right)=\left[r_{\text {wall }}^{*}\left(x^{*}\right) \mid u\left(x^{*}, r^{*}\right)=0.0\right] \\
& -\left[r^{*}\left(x^{*}\right) \mid u\left(x^{*}, r^{*}\right)=0.99\left\|u\left(x^{*}, r^{*}\right)\right\|\right] \\
& \delta^{*}\left(x^{*}\right)=\int_{r_{\text {wall }}^{*}}^{0}\left(1-\frac{\rho\left(x^{*}, r^{*}\right) u\left(x^{*}, r^{*}\right)}{\rho_{0} u_{0}}\right) \mathrm{d} r^{*} \\
& \theta^{*}\left(x^{*}\right)=\int_{r_{\text {wall }}^{*}}^{0} \frac{\rho\left(x^{*}, r^{*}\right) u\left(x^{*}, r^{*}\right)}{\rho_{0} u_{0}}\left(1-\frac{u\left(x^{*}, r^{*}\right)}{u_{0}}\right) \mathrm{d} r^{*} \\
& H\left(x^{*}\right)=\frac{\delta^{*}\left(x^{*}\right)}{\theta^{*}\left(x^{*}\right)}
\end{aligned}
$$

with the approximation of a negligible wall curvature in the azimuthal direction, i.e. $\delta_{b l}^{*} \ll r_{w a l l}^{*}$. The momentum thickness variations close to the step and the nozzle exit are not relevant since the boundary layer is detached at these locations. In the neighborhood of the throat, it get smaller due to the boundary layer thinning. Downstream in the divergent, the metrics $\delta_{b l}^{*}$ and $\theta^{*}$ grow linearly which indicates that the boundary layer is developing. A number of grid points across the boundary layer from 5 at the throat to 15 at the separation point is estimated in that region. The shape factor $H$ takes high values induced by the supersonic convergent-divergent configuration. Indeed, for a standard Blasius boundary layer profile, $H=2.59$ is considered laminar while $H \leq 1.4$ is typically turbulent. ${ }^{56}$ For fully turbulent boundary layers in high-speed compressible flows, the typical shape factor has been found to increase with the Mach number. For instance, Maeder et $a l^{57}$ have carried out a DNS of a boundary layer on a flat plate at three mean flow Mach numbers from $M_{\infty}=3$ to 6 and obtained turbulent shape factors from $H=5.86$ to 17.20 . In the present situation, $H$ therefore gradually increases downstream of the throat because of the flow acceleration. A value of $H \simeq 7$ is obtained at the nozzle exit where $M_{j}=3.1$, which is probably not a fully turbulent condition. However, the geometrical tripping does not aim at providing such an exit condition but only sufficient velocity fluctuations to ensure the early shear layer destabilization at the lips. As shown by the Figure $6 \mathrm{a}$, this requirement is met in practice despite the relaminarization phenomenon. 


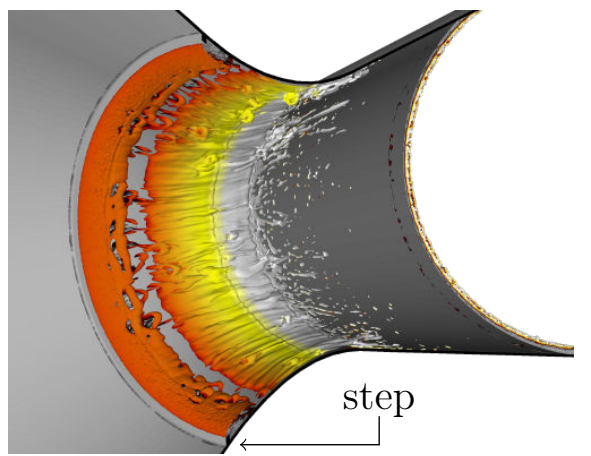

(a)

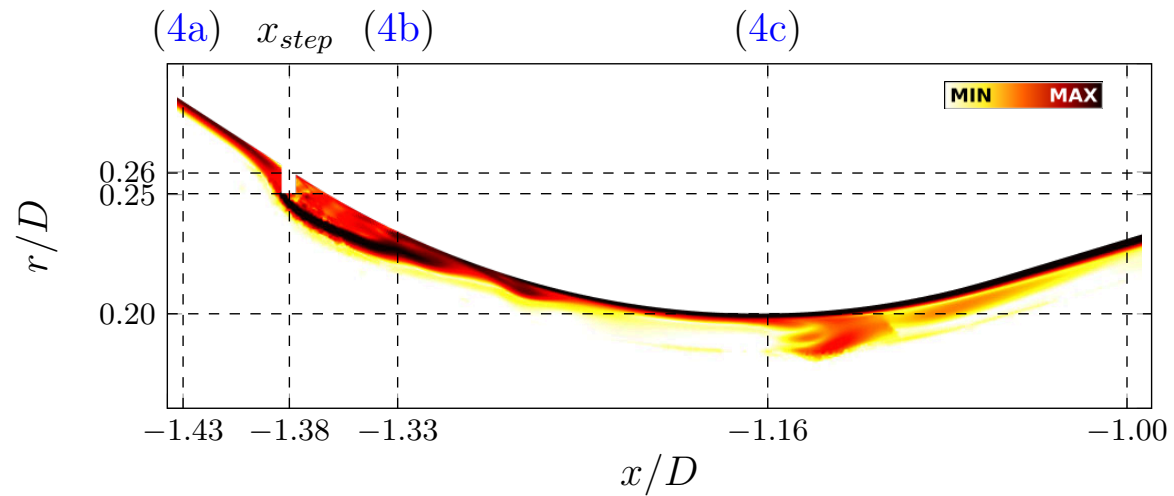

(b)

FIG. 3: flow snapshots in the vicinity of the step. (3a) visualization of iso-Q-criterion surfaces colored by the velocity magnitude and (3b) $2 \mathrm{D}$ visualization of the vorticity intensity.

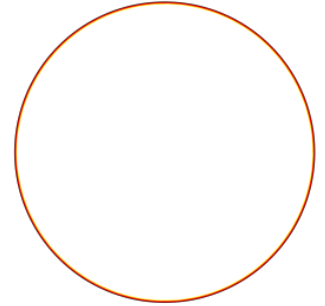

(a)

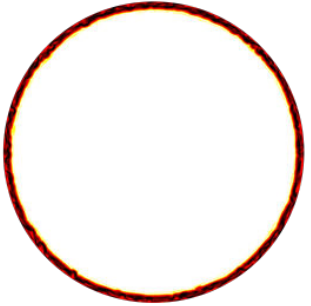

(b)

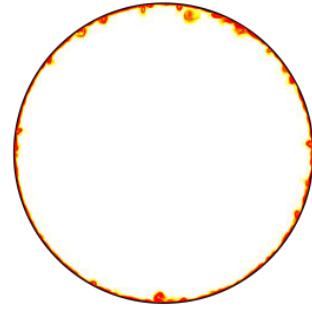

(c)

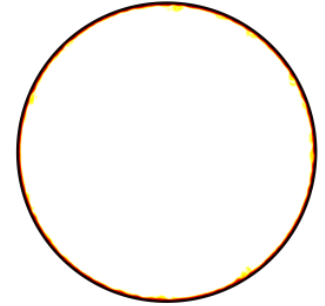

(d)

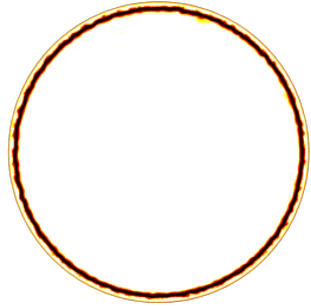

(e)

FIG. 4: dimensionless transversal cut of vorticity in the nozzle at various axial locations. (4a) $x=-1.43 D$, (4b) $x=-1.33 D,(4 \mathrm{c}) x=-1.16 D,(4 \mathrm{~d}) x=-0.50 D,(4 \mathrm{e}) x=0 D$.

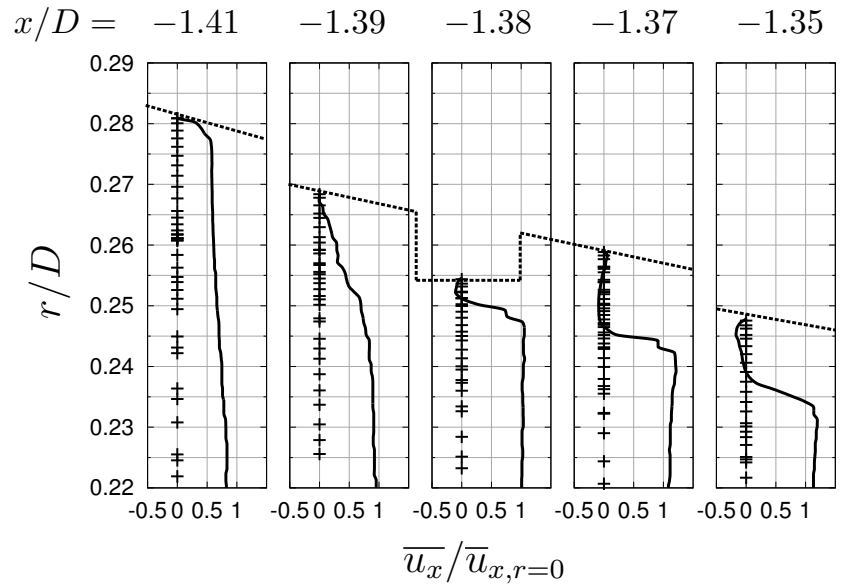

(a)

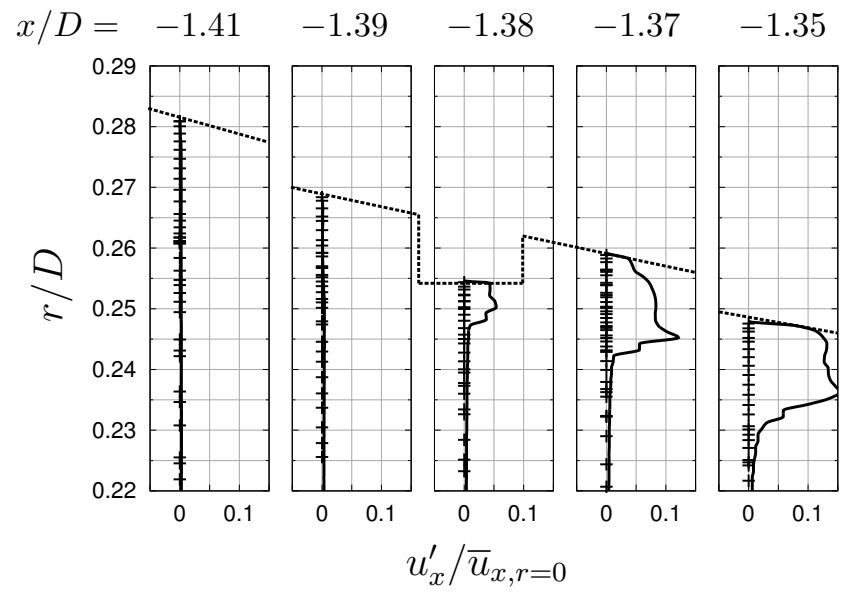

(b)

FIG. 5: velocity properties in the vicinity of the step. (5a) mean axial velocity and (5b) fluctuating axial velocity. -: numerical data; -.-: nozzle wall, +: unstructured mesh discretization every 2 points. 


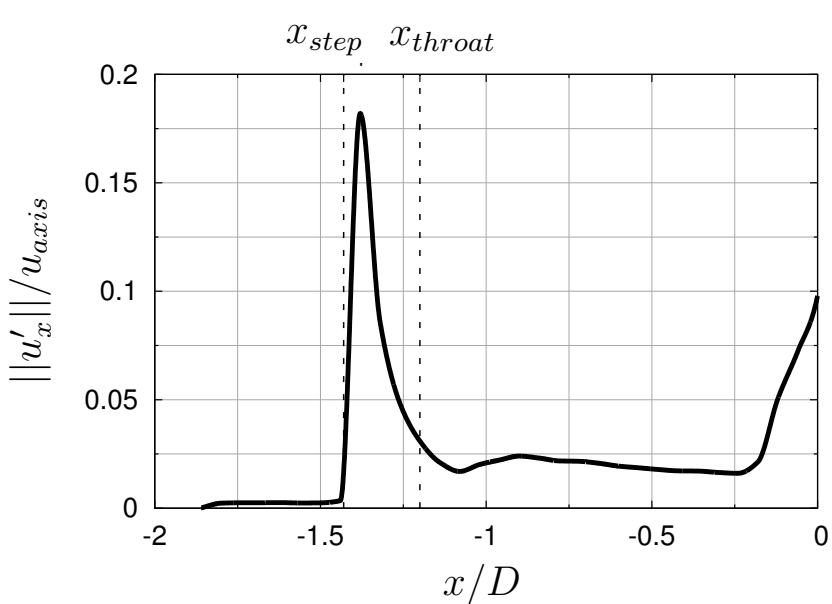

(a)

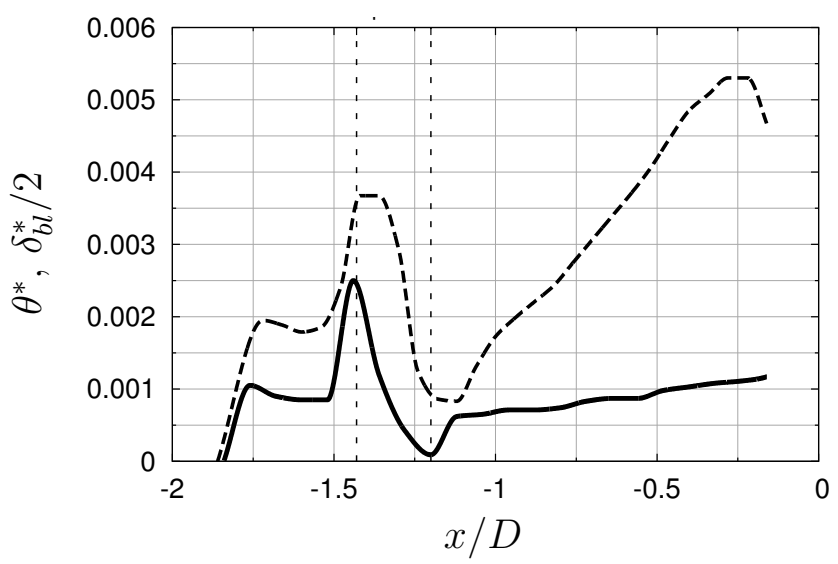

(c)

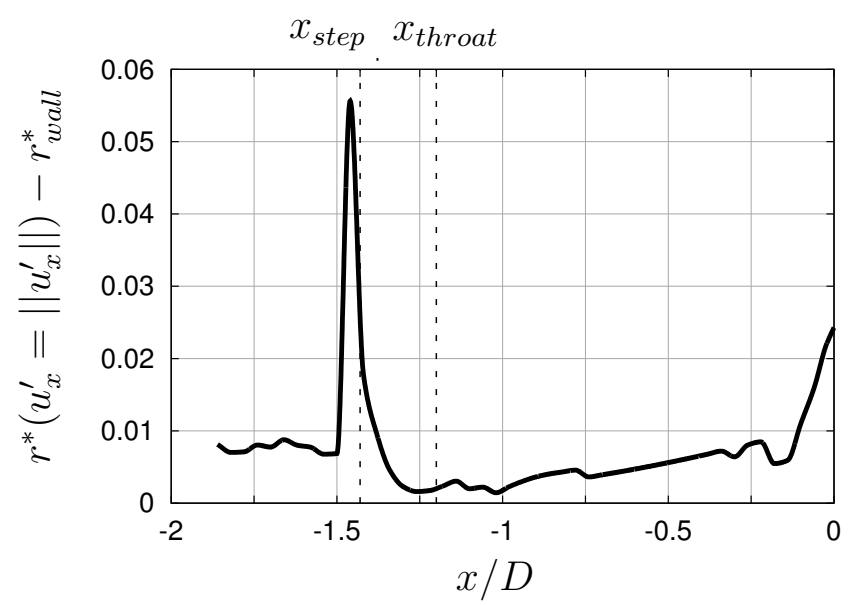

(b)

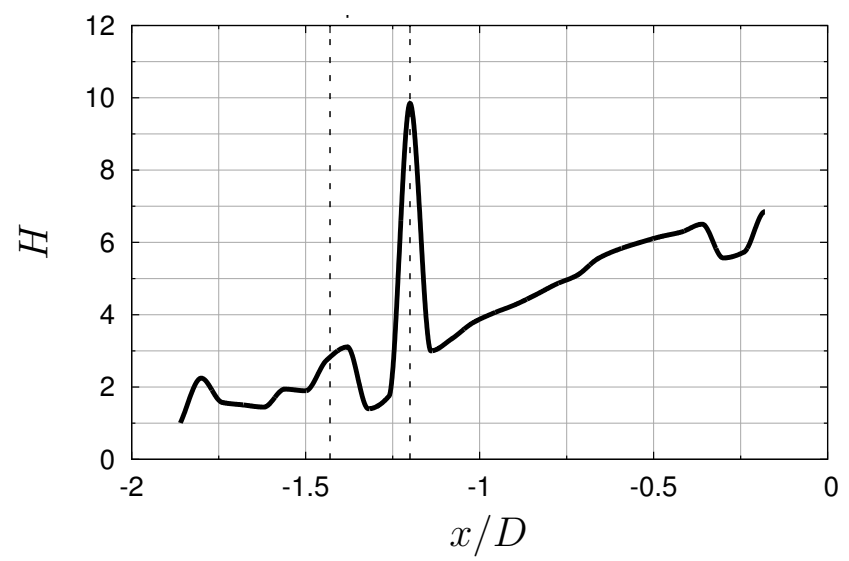

(d)

FIG. 6: nozzle wall boundary layer properties. (6a) maximum of fluctuating velocity; (6b) radial location of the maximum of fluctuating velocity; (6c) - momentum thickness $\theta^{*}$ and $\boldsymbol{- \cdots} \cdot$ : boundary layer thickness $\delta_{b l}^{*}$;

(6d) shape factor $H$. The quantities $\theta^{*}, \delta_{b l}^{*}$ and $H$ are not defined in the separated flow region, i.e. $x>-0.17 D$.

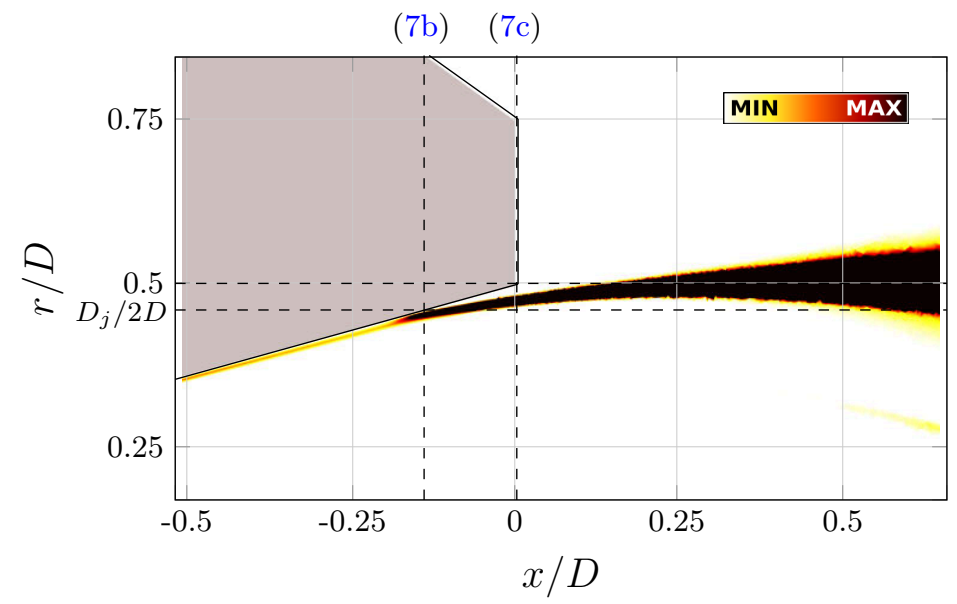

(a)

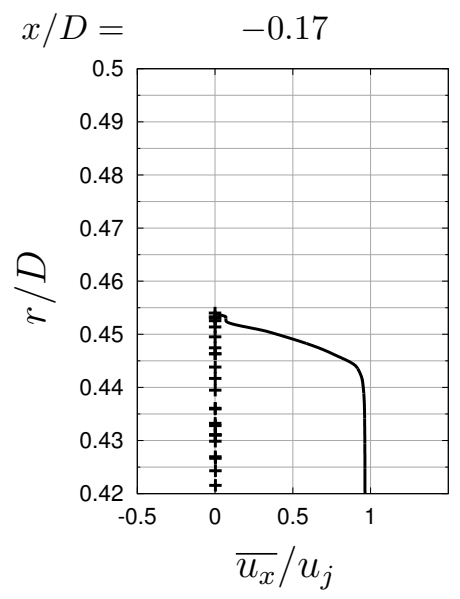

(b)

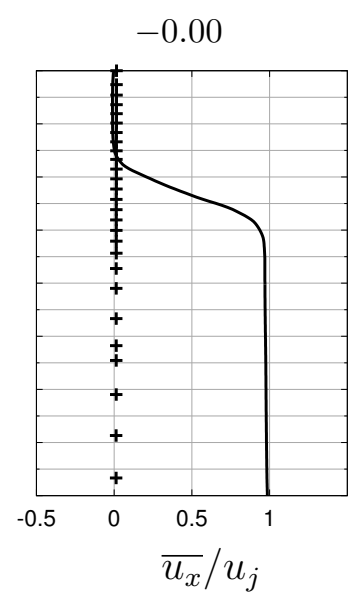

(c)

FIG. 7: mean flow in the vicinity of the separation point. (7a) visualization of $u_{x}$ RMS field, $(7 \mathrm{~b}) \overline{u_{x}}$ radial profile at $x=-0.17 D$ (separation point) and (7c) $\overline{u_{x}}$ radial profile at $x=-0 D$ (nozzle exit). - numerical data; + : unstructured mesh discretization every 2 points. 


\section{SIMULATION VALIDATION}

The full free jet computation is validated in this part, with focuses on the aerodynamic in section III A and the acoustic in section III B. The data from the present simulation is compared to available measurements from the MARTEL facility, previous computations, data from the literature, semi-empirical models and analytical laws in order to estimate its accuracy and predictivity.

\section{A. Aerodynamic}

\section{Snapshots and mean fields visualization}

Mean flow fields and snapshots are presented in Figures 8 and 9 for a qualitative description of the jet near field. Main numerical recording points and profiles are depicted in Figures 8a and 8b. The dashed lines correspond to LDV measurements while the symbols ( $(\bullet)$ represent cylindrical numerical arrays at $r=0.5 D$ (lipline) denoted array A and $r=2.5 D$ denoted array $\mathrm{B}$. The mean density field is displayed in Figure 8a in colored levels. The ejected combustion gas has a lower density than the ambient air because of the high temperature and the overexpanded condition. The species mixing layer seems to spread linearly. Compressible effects are noticeable through the shock cell structure, as well as on the Mach number field in Figure 8b. The drawn sonic line illustrates that the flow in the shear layer is strongly deviated by the successive expansion fans and compressions of the shock cell structure. Array A is essentially located in a supersonic zone. Instantaneous and mean vorticity fields are given respectively in Figures $8 \mathrm{c}$ and $8 \mathrm{~d}$. They show that the shear layer is destabilizing early and spreading properly which is a major improvement compared to the previous computation. ${ }^{44}$ Downstream, a wide variety of vortical structure sizes can be seen on the transversal snapshots in Figures 8c and 9, which suggests a proper shear layer development. The shear layer periodic deviation dues to the shock cells is particularly visible on the mean vorticity field. Triple-points delimiting a small Mach disk are captured at the initial shocks convergence point inside the potential core, at $x=1.33 D$ (Figure 9b), and appear to generate significant vorticity levels. Such an effect can also be observed in the simulation of Liu et $a l .{ }^{53} \mathrm{In}$ order to make further discussions clearer, the visualized jet structure is sketched in Figure 10. It points out that the shocks are not identically axially located on the centerline and on the lipline.

\section{Centerline properties}

The mean velocity, the velocity fluctuations magnitude, the Mach number and the axial velocity skewness and kurtosis along the jet centerline are plotted in Figure 11. The present results are confronted to the experi- mental measurements and numerical data from previous computations. ${ }^{44}$ The mean velocity in Figure 11a agrees with the experiment in terms of shock locations. The numerical profile shows however a strong undershoot at the first shock, also present at a lesser extent in data from Langenais 2017. ${ }^{44}$ On the other hand, a large difference of turbulence intensity between the computation $(<0.5 \%)$ and the experiment $(>6 \%)$ is highlighted upstream of the first shock in Figure 11b. The implemented geometrical tripping method (see §IID) does not enable to provide fluctuations on the centerline while the actual nozzle input conditions in the experiment result from a turbulent combustion not included in the simulation. That could explain the discrepancies of turbulence levels on the axis before the first shock. Velocity fluctuations on the jet centerline inducing shock oscillations could also explain the smoother mean velocity profiles in the experiment, in addition to possible LDV measurement bias such as particle polydispersity, coalescence and lag. ${ }^{58}$ The turbulence level downstream of the first shock is much higher and agrees with the experiment for $1.33<x^{*}<10$. Then it slowly decreases downstream of the end of the potential core as expected for a fully turbulent jet. The disturbances generated by the triple-points lead to a major improvement compared to previous computations where the simulated levels really started to increase only further downstream for $x^{*}>5$.

Based on Figures 11a and 11c, the dimensionless length of the first shock cell on the centerline is estimated to $L_{1}^{*}{ }_{\text {st }}$ shock $=2.6$ for both the experiment and the simulation. It can be approximated with the Tam and Tanna's model ${ }^{59}$ derived from the instability wave theory:

$$
L_{1}^{*}{ }_{\text {st } \text { shock }}=K \pi\left(M_{d}^{2}-1\right)^{0.5} \frac{D_{d}}{\mu_{1} D_{j}}
$$

where $M_{d}=2.8$ is the equivalent ideally-expanded jet Mach number at the exit diameter $D_{d}=51 \mathrm{~mm}, \mu_{1}=$ 2.405 and $K$ a correction constant. Initially set to $K=1$, Tam et al. $^{60}$ recently recommended to take $K=0.9$ which yields $L_{1 \text { st shock }}^{*}=2.6$ and matches with the simulation. The dimensionless supersonic region mean length can be estimated with the Mach number profile on the centerline, leading to $L_{\text {sonic }}^{*} \simeq 21$. It is slightly longer than in the previous computations.

The skewness $S$ and kurtosis $K$ are defined for a discrete signal $s$ by

$$
S(s)=\frac{\overline{s^{3}}}{\sigma^{3}}=\frac{\frac{1}{N} \sum_{i}\left(s_{i}-\bar{s}\right)^{3}}{\left[\sqrt{\frac{1}{N} \sum_{i}\left(s_{i}-\bar{s}\right)^{2}}\right]^{3}}
$$

and

$$
K(s)=\frac{\overline{s^{4}}}{\sigma^{4}}=\frac{\frac{1}{N} \sum_{i}\left(s_{i}-\bar{s}\right)^{4}}{\left[\sqrt{\frac{1}{N} \sum_{i}\left(s_{i}-\bar{s}\right)^{2}}\right]^{4}}
$$

where $\overline{s^{3}}$ and $\overline{s^{4}}$ are the third and fourth statistical moments, $\bar{s}$ the mean, $\sigma$ the standard deviation and $N$ the 


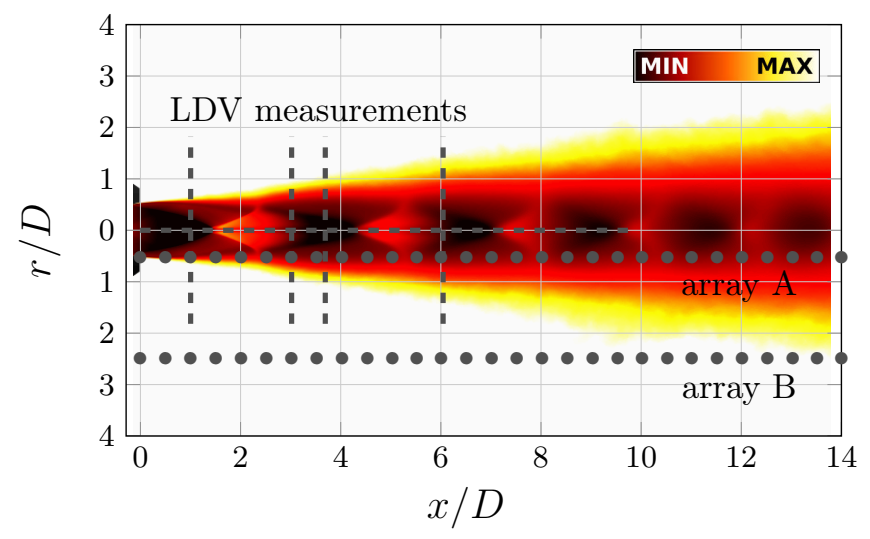

(a)

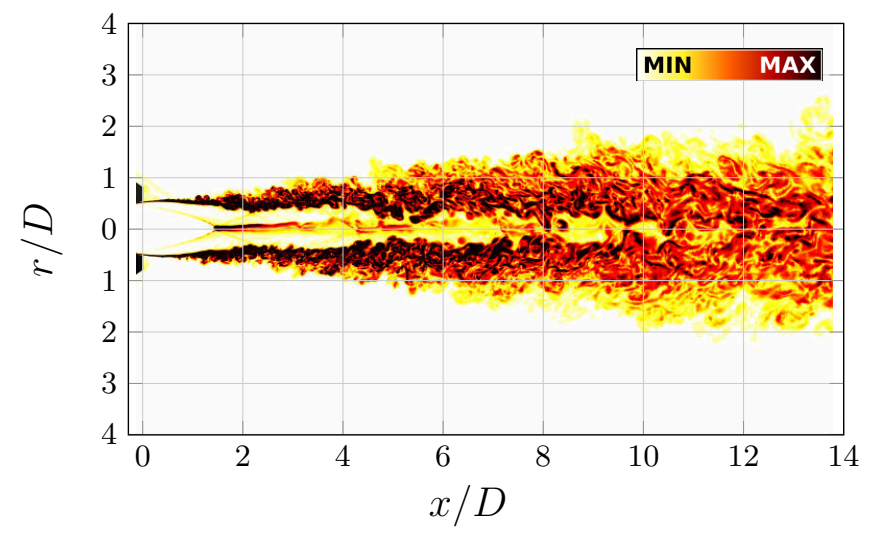

(c)

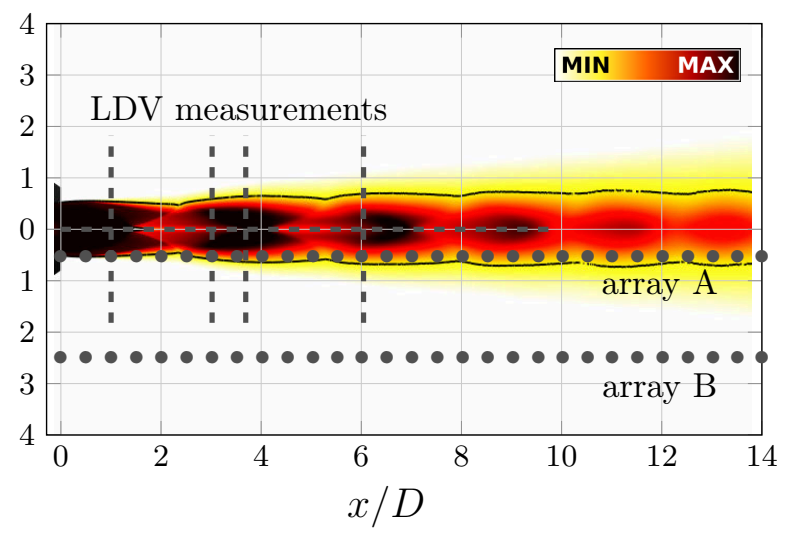

(b)

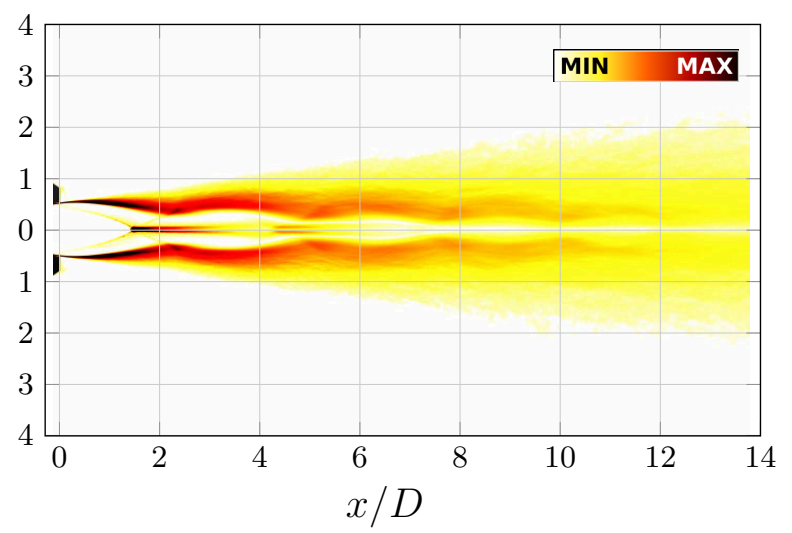

(d)

FIG. 8: visualizations of (8a) mean density field, (8b) mean Mach number field, (8c) instantaneous vorticity field and (8d) mean vorticity field. ๑: numerical arrays; =--: LDV measurement locations; —: sonic line $M=1$.

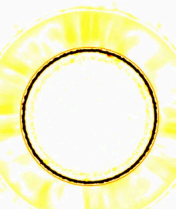

(a)

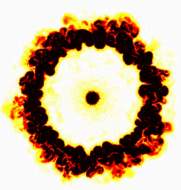

(b)

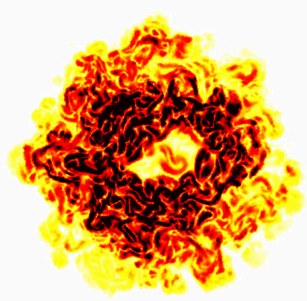

(c)

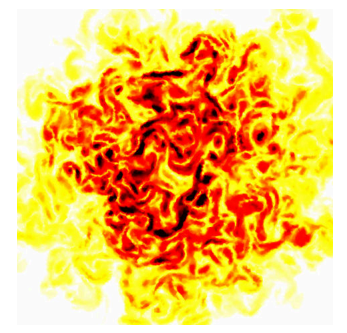

(d)

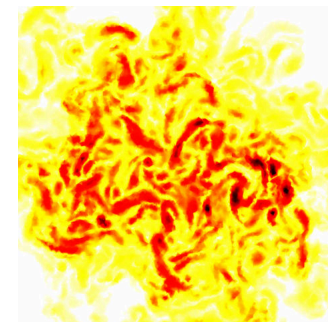

(e)

FIG. 9: dimensionless transversal cut of vorticity in the free jet region at various axial locations.

(9a) $x=0.0 D,(9 \mathrm{~b}) x=1.33 D,(9 \mathrm{c}) x=5.0 D,(9 \mathrm{~d}) x=10.0 D$, (9e) $x=15.0 D$.

number of samples of the signal. These metrics respectively measure the asymmetry and the tailedness of the signal probability distribution, and are equal to $S(s)=0$ and $K(s)=3$ in case of a Gaussian distribution. The skewness and kurtosis of the axial velocity along the centerline are plotted in Figure 11d. The main experimental trends are better recovered than previously at the right locations. The negative peaks of $S$ and positive peaks of $K$ at $x^{*}=6.2$ and $x^{*}=7.5$ are captured. Levels are however not in accordance with the LDV measurements, especially $K$. It could be explained by the fact that these metrics are sensitive to the signal length and the sampling rate ${ }^{43}$ and that the turbulence properties are not similar on the jet axis in the simulation and the experiment as mentioned above. 


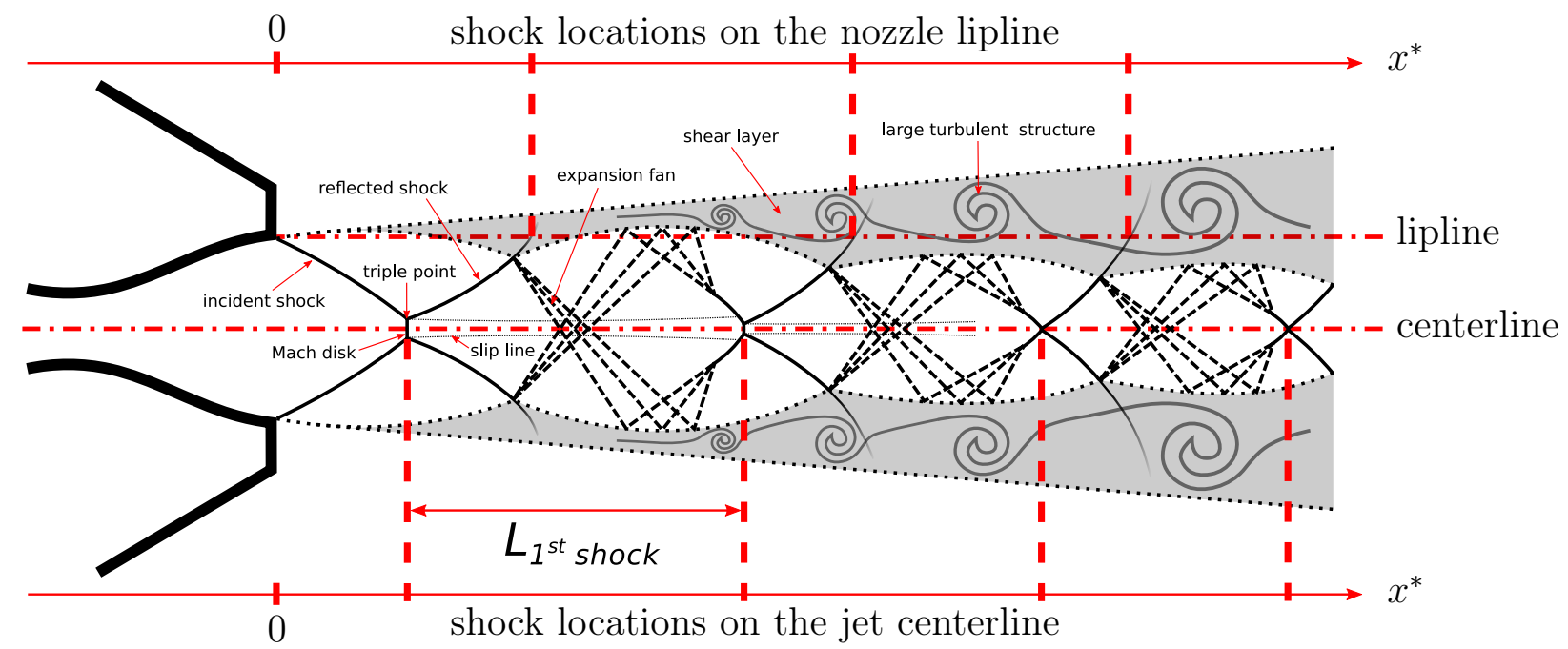

FIG. 10: sketch of the overexpanded jet structure based on the field visualizations in Figure 8.

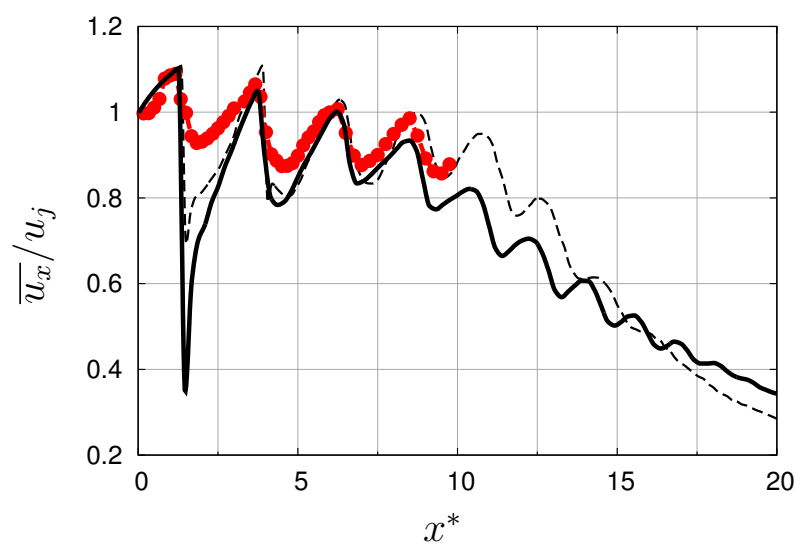

(a)

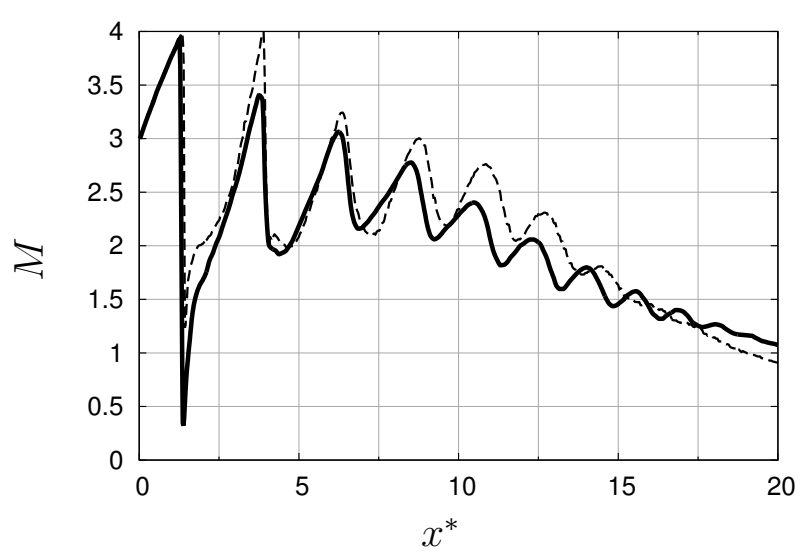

(c)

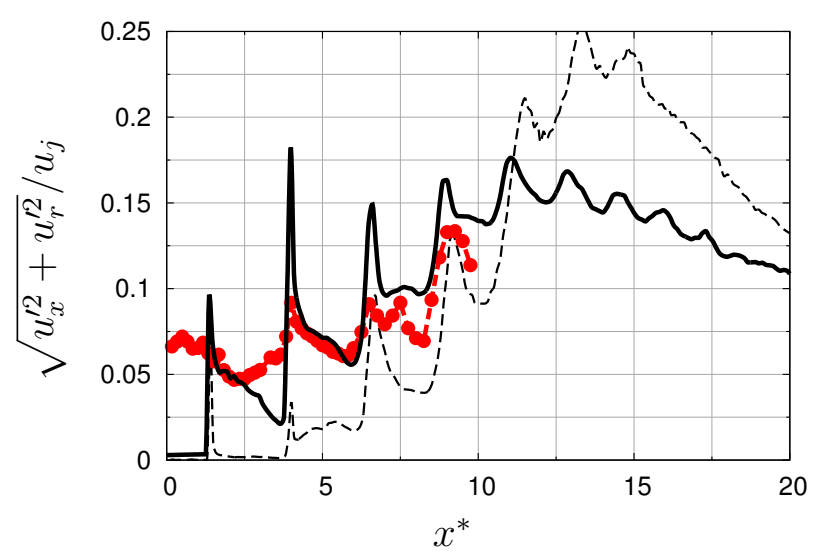

(b)
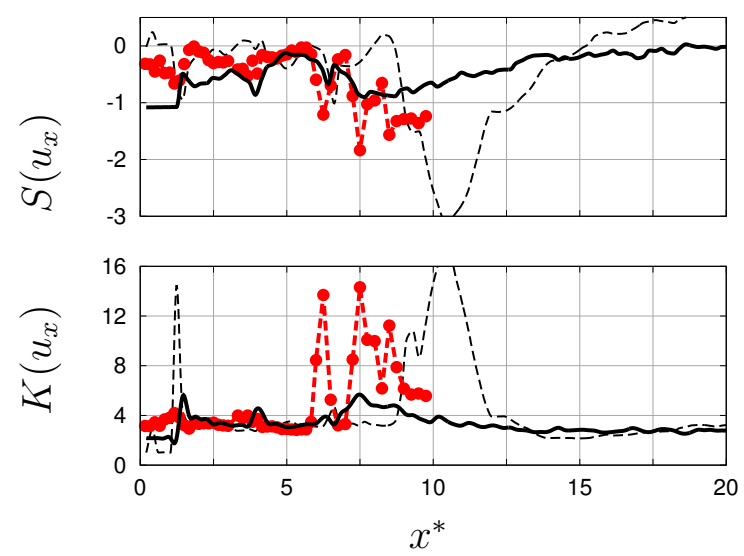

(d)

FIG. 11: velocity properties along the jet centerline. (11a) mean axial velocity, (11b) fluctuating velocity, (11c) axial Mach number $\overline{u_{x}}\left(x^{*}\right) / \bar{c}\left(x^{*}\right)$ where $\bar{c}\left(x^{*}\right)$ is the local mean speed of sound, (11d) axial velocity skewness and kurtosis. --๑-- : experimental data; - : present numerical data; ---: numerical data from Langenais $2017 .^{44}$ 


\section{Shear layer development}

Experimental data from the MARTEL facility includes radial profiles of mean and fluctuating velocity at the four axial locations $x^{*}=1,3,3.67$ and 6 . They are compared to the previous and the present simulation results in Figure 12. Mean profiles of axial (Figure 12a) and radial (Figure 12c) velocity from the present simulation exhibit a significant improvement compared to the previous simulation. The turbulence tripping results in a major benefit in terms of velocity fluctuation levels and shear layer development as reported in Figures 12b and $12 \mathrm{~d}$. The numerical profiles of fluctuating radial velocity show the larger deviation from measurements while levels are globally recovered. The agreement is particularly satisfactory for the fluctuating axial velocity.

The half velocity jet radius $\delta_{0.5}^{*}$, the shear layer thickness $\delta_{s l}^{*}$ and the incompressible shear layer momentum thickness $\theta_{s l}^{*}$ can be calculated from the velocity profiles according to

$$
\begin{gathered}
\delta_{0.5}^{*}\left(x^{*}\right)=r\left(x^{*}\right) \mid u\left(x^{*}, r^{*}\right)=0.5 u\left(x^{*}, 0\right) \\
\begin{aligned}
& \delta_{s l}^{*}\left(x^{*}\right)=\left[r\left(x^{*}\right) \mid u\left(x^{*}, r^{*}\right)=0.05 u\left(x^{*}, r_{0}\left(x^{*}\right)\right)\right] \\
&- {\left[r\left(x^{*}\right) \mid u\left(x^{*}, r^{*}\right)=0.95 u\left(x^{*}, r_{0}\left(x^{*}\right)\right)\right] } \\
& \theta_{s l}^{*}\left(x^{*}\right)=\int_{r_{0}\left(x^{*}\right)}^{\infty} \frac{u\left(r^{*}, x^{*}\right)}{u_{0}}\left(1-\frac{u\left(r^{*}, x^{*}\right)}{u_{0}}\right) \mathrm{d} r^{*}
\end{aligned}
\end{gathered}
$$

where the lower bound $r_{0}\left(x^{*}\right)$ is arbitrarily set to make the integrated values free from the shock cell presence. The incompressible approximation is a priori not suited for this flow. However, the density measurements are not available in the experimental database. The thicknesses are consequently calculated assuming $\rho\left(x^{*}, r^{*}\right)=\rho_{0}$ for comparison purposes. These quantities, as well as the magnitude of $u_{x}^{\prime} / u_{j}$, are plotted along the lipline (array A) in Figure 13. The simulation is found to agree with the experimental data. The fluctuation levels along array $\mathrm{A}$ in Figure $13 \mathrm{~b}$ are similar, for example $u_{x}^{\prime} / u_{j} \simeq 0.15$ at $x^{*}=1$. The nearly null initial value is due to the fact that the closest recording points to the lips on array $A$ are located outside the shear layer knowing that the flow is separated. The numerical asymptotic spread rate of the shear layer is $\mathrm{d} \delta_{s l}^{*} / \mathrm{d} x=0.13$ as drawn in Figure $13 \mathrm{c}$ which is close to the estimated experimental value 0.11 . In the same way, the asymptotic spread rate of the momentum is found to be $\mathrm{d} \theta_{s l}^{*} / \mathrm{d} x=0.022$ numerically and 0.020 experimentally. These values are consistent with the experimental results of Troutt and McLaughlin ${ }^{61}$ studying a $M_{j}=2.1, R e_{D} \sim 10^{6}$ jet, reported on Figure 13c, or Hussain and Zedan ${ }^{26}$ studying initially disturbed laminar shear layers. The latter have found spread rates of $\mathrm{d} \delta_{s l}^{*} / \mathrm{d} x=0.16$ and $\mathrm{d} \theta_{s l}^{*} / \mathrm{d} x=0.029$ for a shear layer with an initial velocity fluctuation intensity $u^{\prime} / u_{j}=0.08$ and have shown that these values decrease by increasing $R e_{D}$ or decreasing $u^{\prime} / u_{j}$. They have also concluded that the self-preserving zone, i.e. with a linear spread rate, is quickly reached downstream of the separation point as obtained in the present simulation.

The dimensionless potential core length $L_{\text {core }}^{*}$ is estimated considering the half velocity jet width $\delta_{0.5}^{*}$ in Figure 13a and the shear layer width $\delta_{s l}^{*}$ in Figure 13c. This length is defined as the axial location for which $\delta_{s l}^{*}=2 \delta_{0.5}^{*}$ leading to $L_{\text {core }}^{*} \simeq 8$ and is reported in Figure 13. It is found shorter than in the previous computation. This value also appears to deviate with most of the empirical models known in the literature such as the formulas of Nagamatsu and Horvay, Eldred or Lau. Many authors found that the potential core shortens while increasing the jet temperature. According to Greska et al. ${ }^{62}$ these models are not validated for hot supersonic jets and cannot readily take into account the temperature effect via the only dependence on the Mach number. Greska et $a l{ }^{62}$ consequently proposed a new formulation for hot jets with an exponential correction term,

$$
L_{\text {core }}^{*}=3.134 \exp \left(1.043 M_{d}-M_{c}\right) \times \frac{D_{d}}{D_{j}}=8.6
$$

where $M_{c}$ is the convective Mach number defined by $M_{c}=u_{j} /\left(c_{j}+c_{\infty}\right)=1.9$. This model is in better agreement with the simulation. However, the faster decrease of the centerline velocity in Figure 11a and the small overestimation of the shear layer spread rate in Figure $13 \mathrm{c}$ regarding the experimental data suggest that $L_{\text {core }}^{*}$ could be still underestimated in the simulation.

The shear layer shows realistic features and the turbulent structures seems to properly develop in Figures 8c and 9. Azimuthally averaged PSDs of the axial velocity are plotted for different $x^{*}$ along array A in Figure 14 in order to quantitatively check that the turbulence have correct physical properties. Broadband spectra can be seen in Figure 14a without significant peaky values. The levels quickly drop at frequencies $S t \geq 0.1$ according to a $S t^{-5 / 3}$ slope for all spectra which indicates a proper turbulent energy cascade. The cut-off frequency of the flow solution is reduced when moving downstream due to the mesh coarsening but remains higher than the expected value $S t_{c}=0.3$ in the acoustic near field (see §II C 2). The velocity spectra at all axial locations on array $\mathrm{A}$ are presented as a spectrogram function of $x^{*}$ in Figure 14b. The shock cells have an important effect on the fluctuation spectra. The intensity rises and reaches a maximum just downstream of the shock before falling till the next shock. This phenomenon is particularly noticeable for the first two shock cells at high frequencies. It can be associated with both the shock/turbulence interaction amplification effect ${ }^{63}$ and the shear layer deviation pointed out in section III A 1 (see Figure 8d). The spectrogram also highlights that mid frequency fluctuations are dominant for $x^{*}<L_{\text {core }}^{*}$ while low frequency fluctuations associated with large turbulent structures are prevalent downstream. 


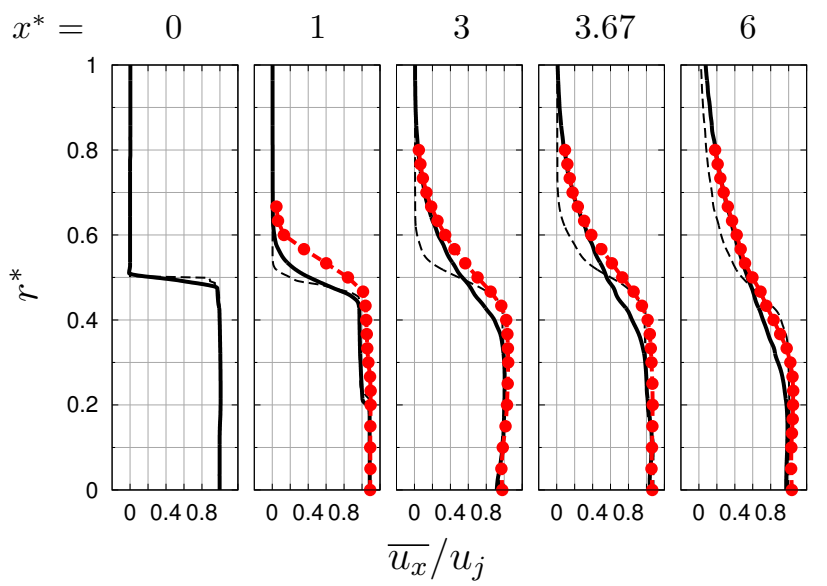

(a)

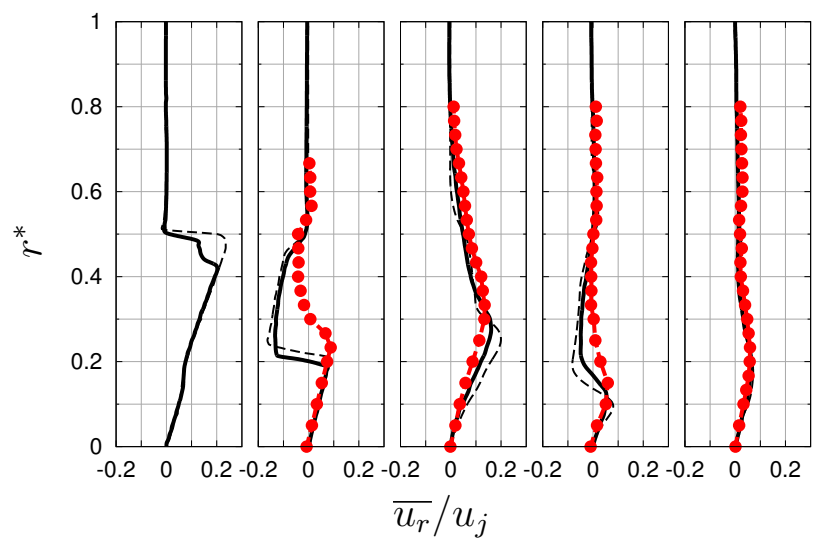

(c)

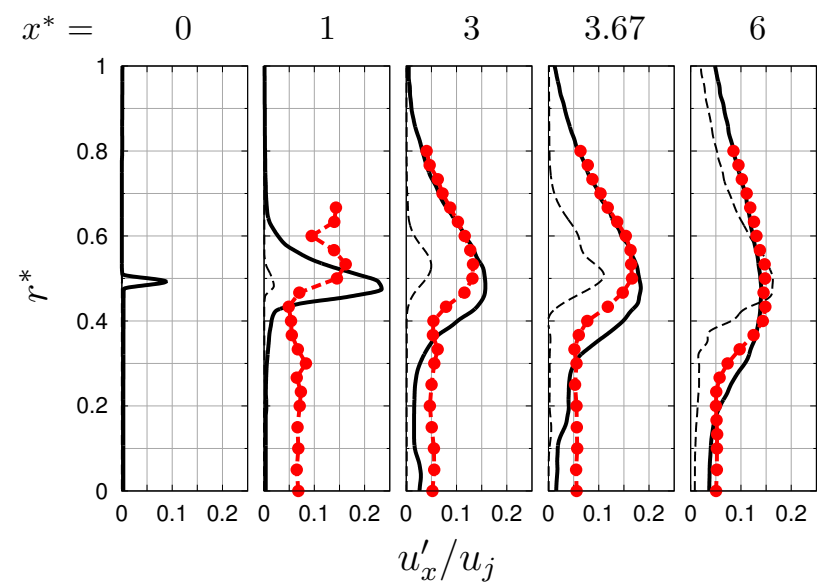

(b)

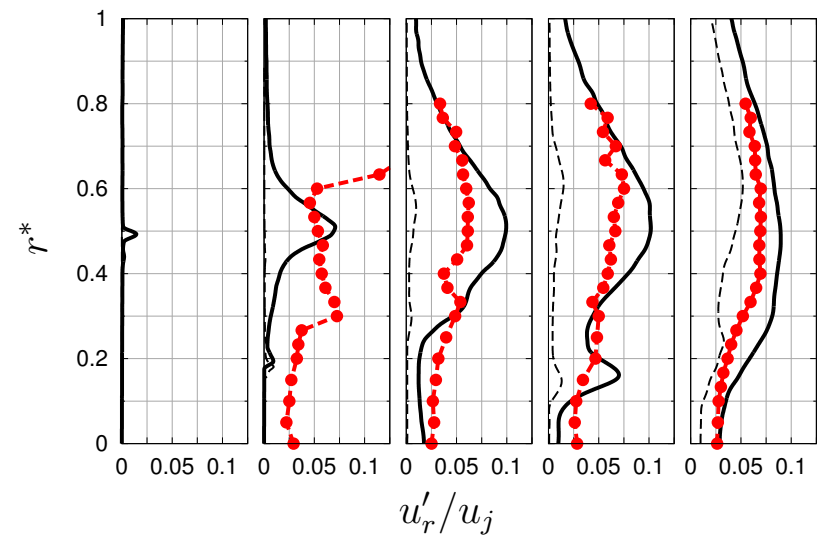

(d)

FIG. 12: radial profiles of mean (12a) axial and (12c) radial velocity and fluctuating (12b) axial and (12d) radial velocity. --๑--: experimental data; —: present numerical data; ---: numerical data from Langenais 2017. ${ }^{44}$

\section{B. Acoustic}

\section{Snapshots visualization}

The agreement of the flow solution with the experimental measurements and other available data suggests that the noise generation is properly predicted. Snapshots of the density and pressure fields in Figure 15 do not show any discontinuity at the coupling interface. The acoustic waves seems to be propagated without significant numerical damping. The expected peak directivity associated with the Mach wave radiation for such a supersonic jet (see §IV A 1) is recovered downstream. No strong vortical structures are found to cross the interface which should avoid any spurious noise generation concern.

\section{Overall levels}

The far field acoustic levels computed by the Euler solver at the microphone locations are given in Figure 16 as a function of the observation angle. The initial angle denoted $\theta$ is centered on the nozzle exit as illustrated in Figure 17. As proposed in other studies on supersonic jet noise, ${ }^{7,64,65}$ an alternative observation angle $\theta^{\prime}$, centered on the end of the potential core, is defined according to the transformation

$$
\theta^{\prime}= \begin{cases}\arctan \left(\frac{\sin (\theta)}{\cos (\theta)-\frac{L_{\theta}}{R_{\theta}}}\right) & \mid \theta<\arccos \left(\frac{L_{\theta}}{R_{\theta}}\right) \\ \arctan \left(\frac{\sin (\theta)}{\cos (\theta)-\frac{L_{\theta}}{R_{\theta}}}\right)+\pi & \mid \theta>\arccos \left(\frac{L_{\theta}}{R_{\theta}}\right)\end{cases}
$$

where $L_{\theta}=L_{\text {core }}=8 \pm 1 D_{j}$ and $R_{\theta}=41.6 D$. Considering an uncertainty of $1 D_{j}$ for $L_{\text {core }}$, the uncertainty propagated to $\theta^{\prime}$ is about $1^{\circ}$. The transformation is illustrated in Figure 17 and reported on the top axis in Figure 16. The overall sound pressure levels (OASPL) are calculated from the pressure signals by integrating the PSDs over a finite frequency range. The experimental and numerical PSDs are integrated over the same range for a fair comparison. The lower bound is set to 


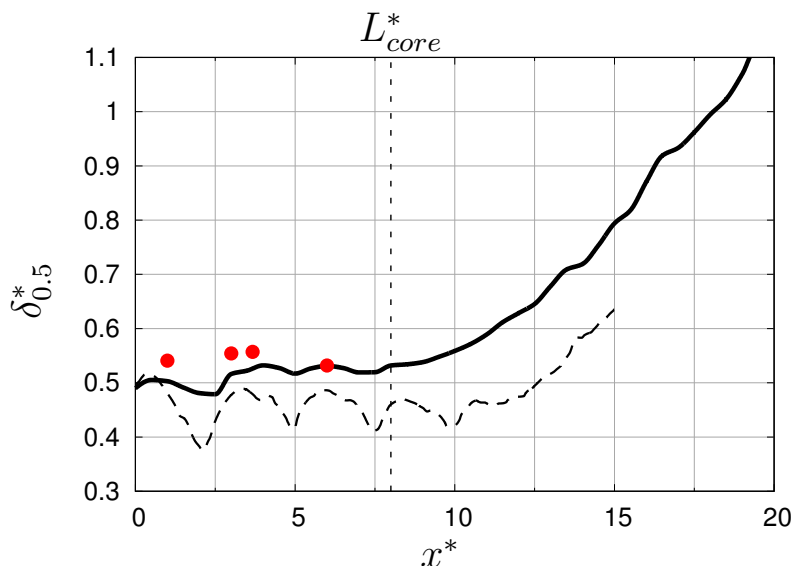

(a)

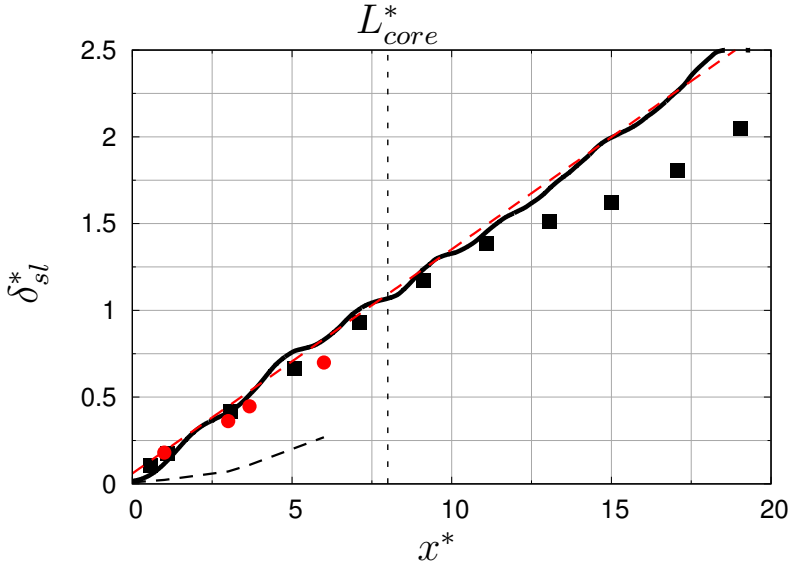

(c)

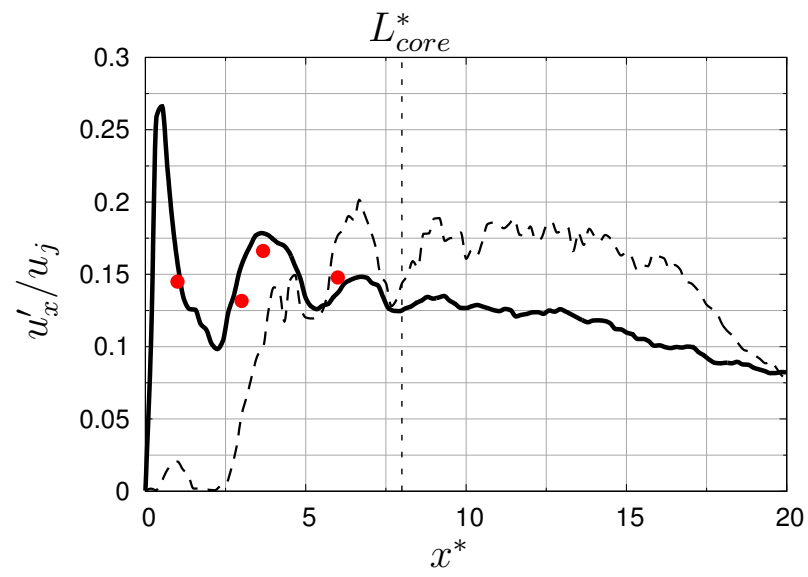

(b)

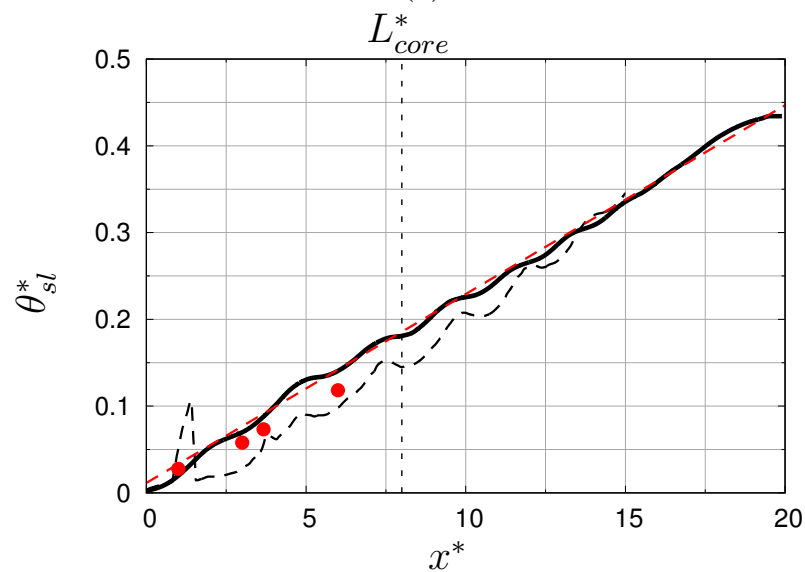

(d)

FIG. 13: free jet shear layer properties. (13a) half velocity thickness, (13b) fluctuating axial velocity along the lipline (array A), (13c) shear layer thickness, (13d) momentum thickness. O: experimental data from MARTEL;

口: experimental data from Troutt and McLaughlin ${ }^{61}\left(M_{j}=2.1, R e \sim 10^{6}\right)$; - : present numerical data; --- : numerical data from Langenais $2017 ;^{44}---$ : asymptotic spread rates $\mathrm{d} \delta_{s l}^{*} / \mathrm{d} x^{*}=0.13$ and $\mathrm{d} \theta_{s l}^{*} / \mathrm{d} x^{*}=0.022$.

$S t=0.01>S t_{\min }$ which is slightly higher than the MARTEL facility absorption devices cut-off estimated to $S t=0.007(\simeq 200 \mathrm{~Hz})$. The upper bound is set to the simulation cut-off $S t_{c}=0.22$.

Considering this frequency bandwidth, the simulation finely agrees with the measurements. It is a major improvement compared to the previous NavierStokes-Ffowcs Williams \& Hawkings computation as depicted in Figure 16. The specific influence of the method (FWH vs Euler) on this result is discussed later in section IV D 3. The slightly higher experimental levels at shallow angles could be due to a numerical underestimation induced by the mesh coarsening in the zone Z1 for $x^{*}>20$ (see $\S I I C 2$ ), or spurious ground reflections in the experiment despite the wedges. Nevertheless, the directivity shape is captured. The numerical peak directivity is located at $\theta_{\text {peak }} \simeq 40^{\circ}$ which corresponds to $\theta_{\text {peak }}^{\prime} \simeq 48^{\circ}$.

\section{Power spectral densities}

The numerical PSDs at the four angles $\theta=30^{\circ}, 60^{\circ}$, $90^{\circ}$ and $120^{\circ}$ are compared to those from the experimental acoustic signals in Figure 18. The PSDs are calculated using the Welch method with a $50 \%$ block overlapping and a natural windowing as recommended for broadband spectra. Experimental and numerical signals are averaged respectively over 1000 and 11 blocks, resulting in frequency resolutions $\Delta S t=0.005$ and 0.01 . The high level broadband spectra at shallow angles are characteristic of Mach wave radiation. The peaks in medium frequencies at larger angles are due to the additional broadband shock-associated noise (see §IV A 2) which becomes dominant in the side and upstream directions and induces a bump on spectra (see Figure 18d). The agreement between the simulation and the experimental data is satisfactory over the resolved frequency bandwidth which indicates that the main features of the 


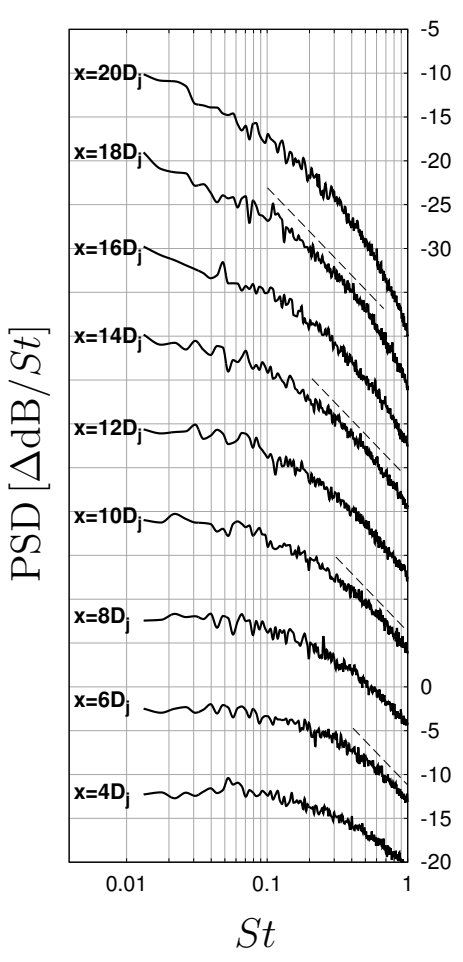

(a)

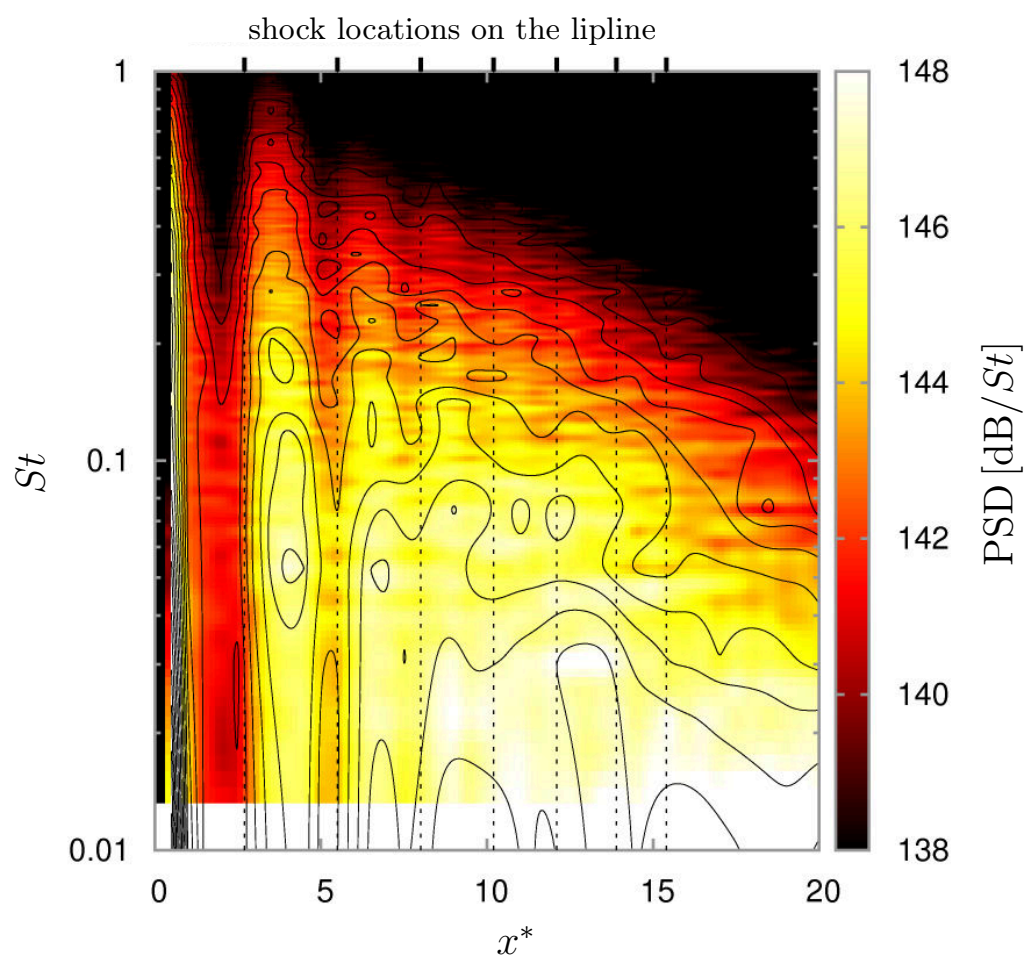

(b)

FIG. 14: PSDs of the axial velocity measured in the shear layer along the lipline (array A) with (14a) PSDs offset by $10 \mathrm{~dB}$ from others and (14b) spectrogram function of $x^{*}$. - : present numerical data; ---: $S t^{-5 / 3}$ slope (Kolmogorov law); —: PSD iso-lines every $\Delta 1 \mathrm{~dB} / S t$.

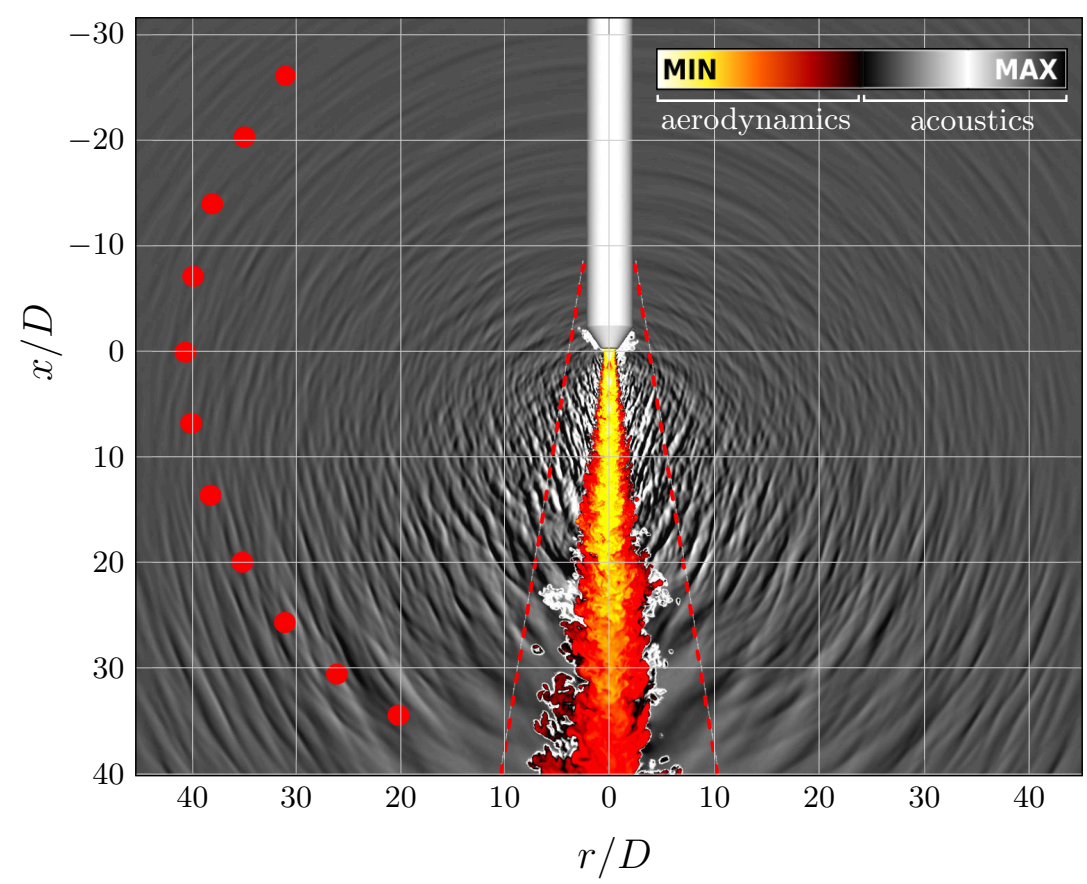

(a)

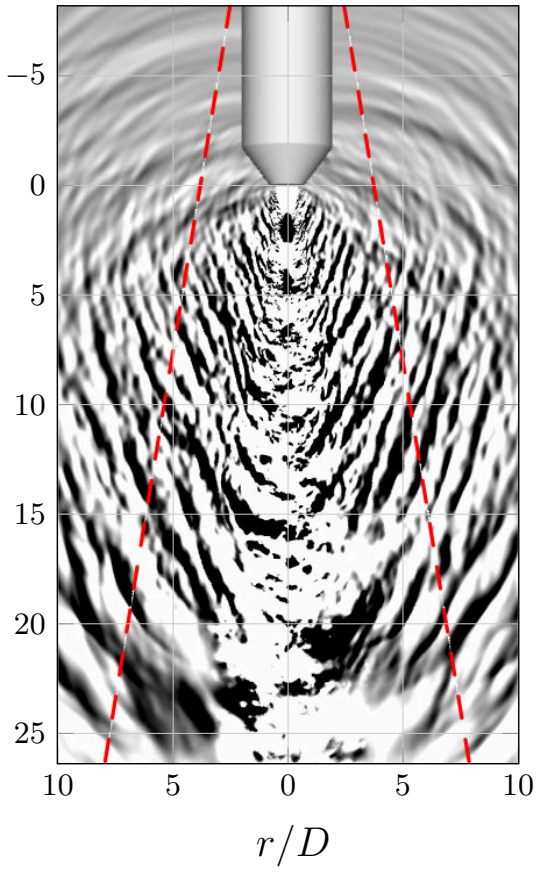

(b)

FIG. 15: visualization of the (15a) density near and far field and (15b) pressure near field. ---: coupling interface; •: far field microphone locations. 


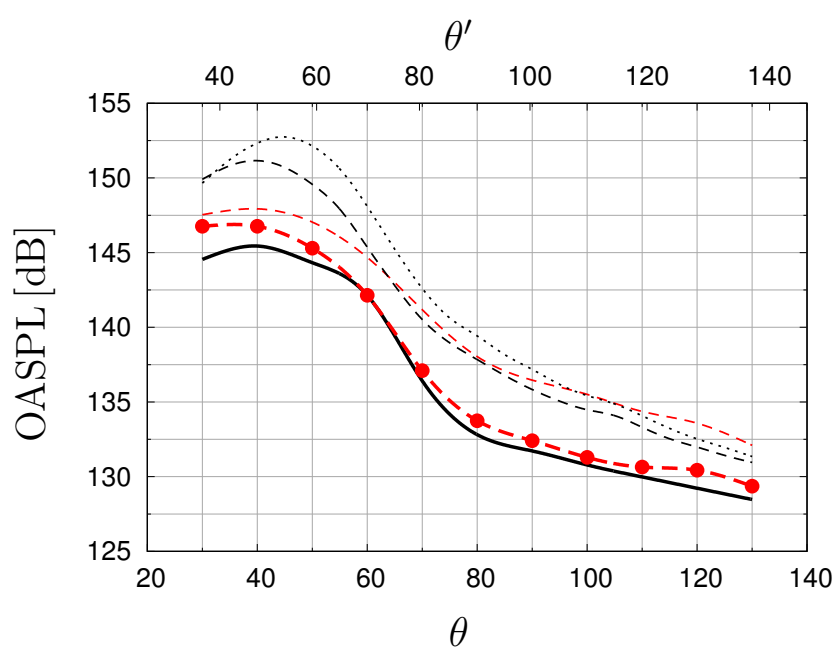

FIG. 16: OASPL at the far field microphone locations function of $\theta$ and $\theta^{\prime}$. Exp. PSDs integrated - -0- : over

the range $0.01 \leq S t \leq 0.22$ and --- : over the full spectrum; - : present numerical PSDs integrated over the range $0.01 \leq S t \leq 0.22 ; \cdots /---$ : numerical PSDs

from Langenais $2017^{44}$ integrated over the range $0.01 \leq S t \leq 0.22$ respectively using the NS-FWH/NS-Euler approach.

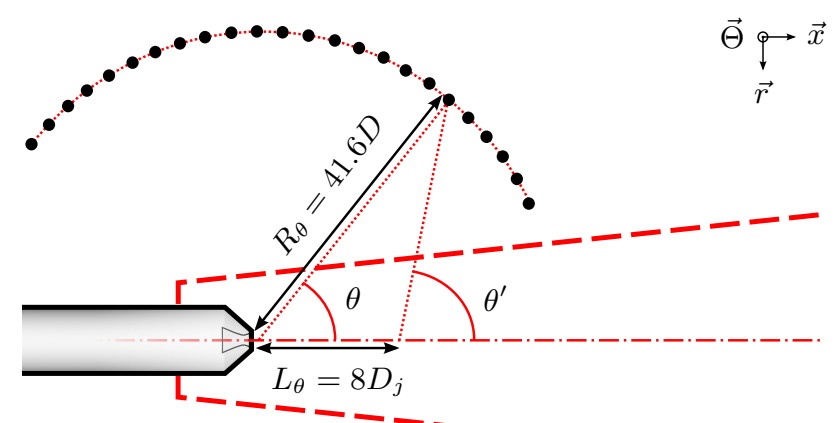

FIG. 17: observation angle transformation from $\theta$ to $\theta^{\prime}$ centered on the end of the potential core.

acoustic field are properly predicted. The effective cutoff frequency is found to be equal to the expected value $S t_{c}=0.22$. The mid frequency levels are finely reproduced for $\theta \geq 60^{\circ}$, including the shock-associated bump. Low frequencies are slightly underestimated in the upstream direction, as high frequencies at $\theta=30^{\circ}$, explaining the previously mentioned level deficit at smallest angles in Figure 16. Differences are also observed at low frequencies $S t<0.02$ which could reveal test bench environmental effects such as spurious low frequency reflections, or a lack of numerical convergence. Finally, a significant part of the dominant acoustic spectra is not properly simulated at high frequencies with respect to the experimental data due to the limited cut-off frequency.

\section{ANALYSIS OF THE RADIATED NOISE}

The agreement of the simulation with most of the available aerodynamic and acoustic data is demonstrated at this point and the results can therefore be relevantly exploited for a physical analysis. The aeroacoustic near and far fields are described and analyzed in this part, with emphasis on the noise sources in section IV A, the resulting pressure near field in section IV B, pressure far field in section IV C and the nonlinear propagation effects in section IV D. Simple and double Fourier transforms, cross-correlations and azimuthal mode decomposition are applied to characterize the noise properties. Specific metrics are then calculated to identify nonlinear effects.

\section{A. Jet noise sources}

The noise from imperfectly expanded supersonic jet is admitted to be generated by three main mechanisms: ${ }^{2,3}$ the convection of large turbulent structures in the shear layer, the mixing of fine turbulent scales in the shear layer and the interaction of turbulent structures with shocks. The first one is associated with the Mach wave radiation and the third one with the broadband shock-associated noise.

\section{Mach waves}

Strong amplitude Mach waves are expected to be generated when large turbulent structures are convected at supersonic speeds relatively to the ambient medium. This mechanism is often described through the wavy wall analogy as shown by Tam. ${ }^{2}$ Three main instability wave families initially observed by Oertel et al. ${ }^{66}$ are expected to produce Mach waves. Their associated convective Mach numbers are

$$
\begin{aligned}
& M_{c 1}=\frac{u_{j}-c_{j}}{c_{j}+c_{\infty}}=1.3 \\
& M_{c 2}=\frac{u_{j}}{c_{j}+c_{\infty}}=1.9 \\
& M_{c 3}=\frac{u_{j}+c_{j}}{c_{j}+c_{\infty}}=2.5
\end{aligned}
$$

Given that the Mach wave appearance conditions $M_{c 2}>$ 0.8 and $M_{c 3}>1.25^{62,67}$ are met and that the convective Mach numbers are all supersonic, the three kinds of instability wave are expected to radiate Mach waves at Mach angles $\theta_{M_{n}}^{\prime}$ defined by

$$
\theta^{\prime}=\arccos \left(\frac{1}{M_{c}}\right)
$$




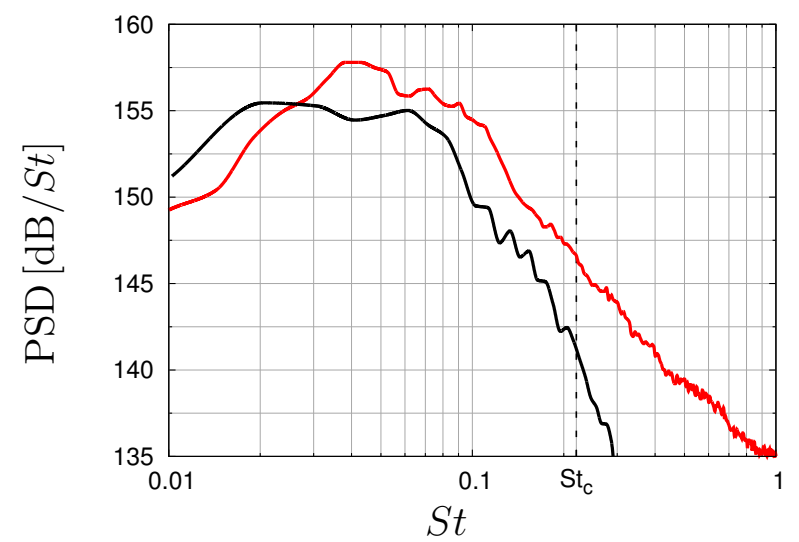

(a)

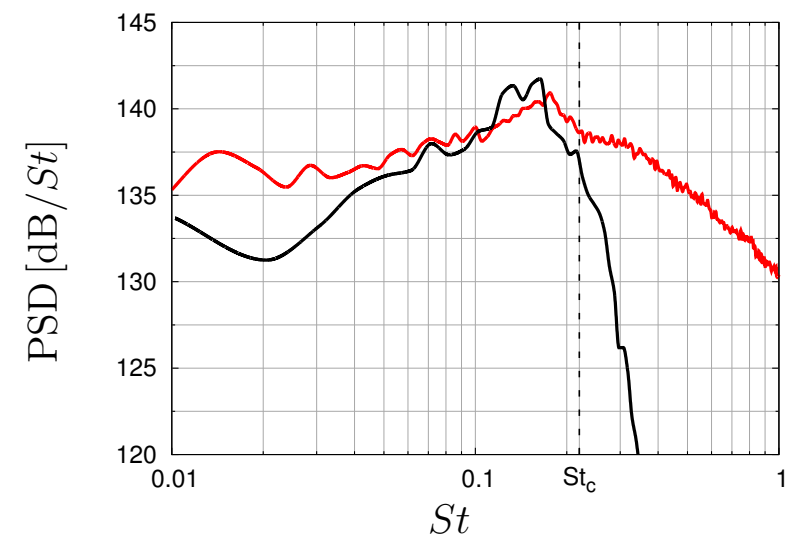

(c)

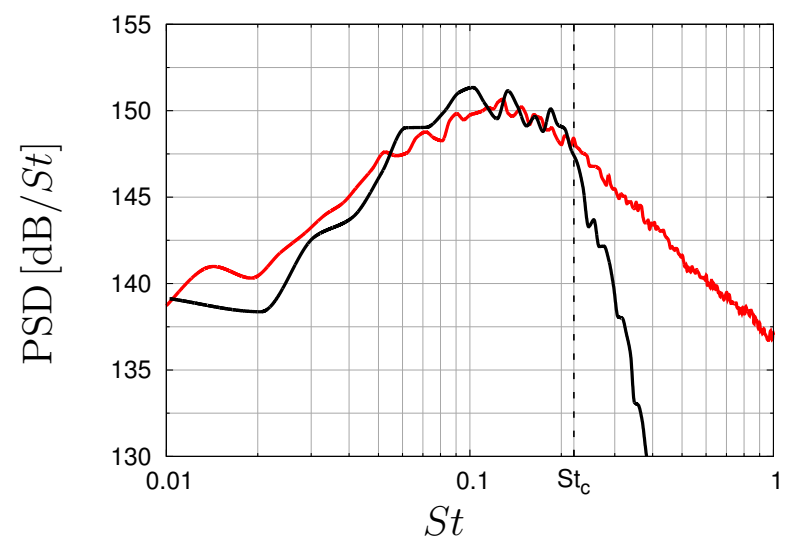

(b)

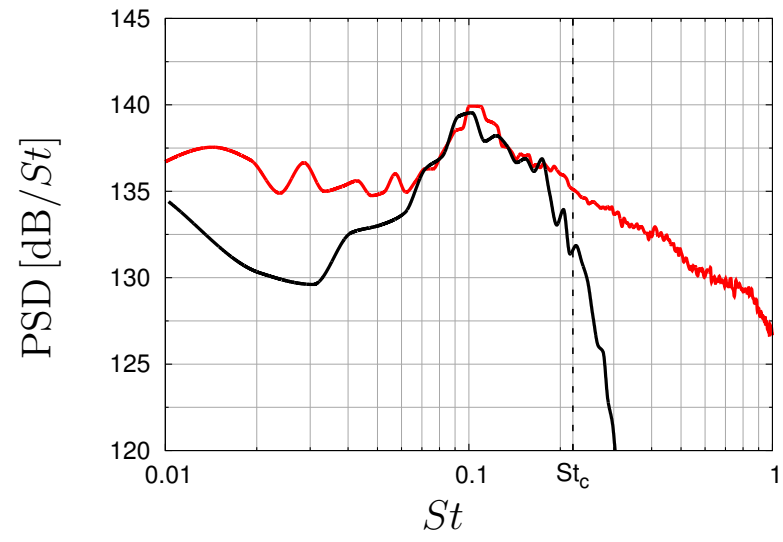

(d)

FIG. 18: PSDs of the pressure at four observation angles. (18a) $\theta=30^{\circ}\left(\theta^{\prime}=36.6^{\circ}\right),(18 \mathrm{~b}) \theta=60^{\circ}\left(\theta^{\prime}=70.4^{\circ}\right)$, (18c) $\theta=90^{\circ}\left(\theta^{\prime}=100.9^{\circ}\right),(18 \mathrm{~d}) \theta=120^{\circ}\left(\theta^{\prime}=128.6^{\circ}\right)$. . $\longrightarrow$ : experimental data; $\longrightarrow$ : present numerical data.

yielding $\theta_{M_{c 1}}^{\prime}=40^{\circ}, \theta_{M_{c 2}}^{\prime}=58^{\circ}$ and $\theta_{M_{c 3}}^{\prime}=66^{\circ}$. The transformed angle system centered on the end of the potential core as defined by equation (14) is used here because it is considered as a better approximation of actual radiation directions. The convection velocities of the turbulent structures with respect to the ambient medium can also be calculated by performing a double fast Fourier transform in time and space, noted $\widehat{p}$ for the pressure, or cross-correlations inside the jet shear layer. These approaches lead to the results depicted in Figure 19. The normalized magnitude of the double FFT function of the dimensionless wave number $k D_{j}$ (transform in space along array A and $S t$ (transform in time) is given in Figure 19a. The maximum of the double FFT seems to follow a slope corresponding to a convection speed $u_{c} / u_{j}=0.43$, i.e. $M_{c}=2.2$ which is reported as a black dashed line. This value represents the most likely convection speed in the shear layer in the range $0 \leq x^{*} \leq 20$ and is close to the slopes deduced from $M_{c 2}$ and $M_{c 3}$ reported as gray dashed lines. This result precisely matches with the unique Mach number, referred to as the Oertel convective Mach number, defined by Greska et al. ${ }^{62}$ as

$$
M_{c o}=\frac{u_{j}+0.5 c_{j}}{c_{j}+c_{\infty}}=2.2
$$

suggesting that this number could be a key parameter of the Mach wave generation mechanism. The corresponding radiation angle is $\theta_{M_{c o}}^{\prime}=63^{\circ}$.

The convection velocity $u_{c}$ versus $x^{*}$ in Figure $19 \mathrm{~b}$ is obtained by cross-correlating the axial velocity recorded at successive probes of array $\mathrm{A}$, spaced by $\Delta x^{*}$, two by two. The convection velocity at a location $x^{*}$ is deduced from the delay of the maximum correlation and the results are azimuthally averaged as

$$
u_{c}\left(x^{*}\right)=\int_{0}^{2 \pi} \frac{\Delta x^{*}}{2 \pi \tau_{\max }\left(x^{*}, \Theta\right)} \mathrm{d} \Theta
$$

given

$$
\begin{aligned}
& {\left[u_{x}\left(x^{*}, t\right) \star u_{x}\left(x^{*}+\Delta x^{*}, t\right)\right]\left(\tau_{\max }\right)} \\
& =\max \left[\left[u_{x}\left(x^{*}, t\right) \star u_{x}\left(x^{*}+\Delta x^{*}, t\right)\right](\tau)\right]
\end{aligned}
$$




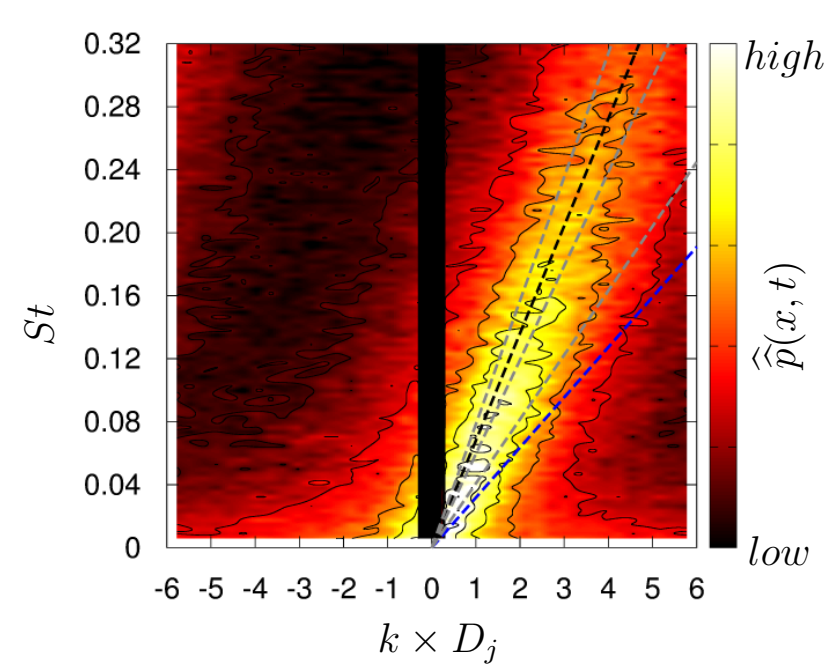

(a)

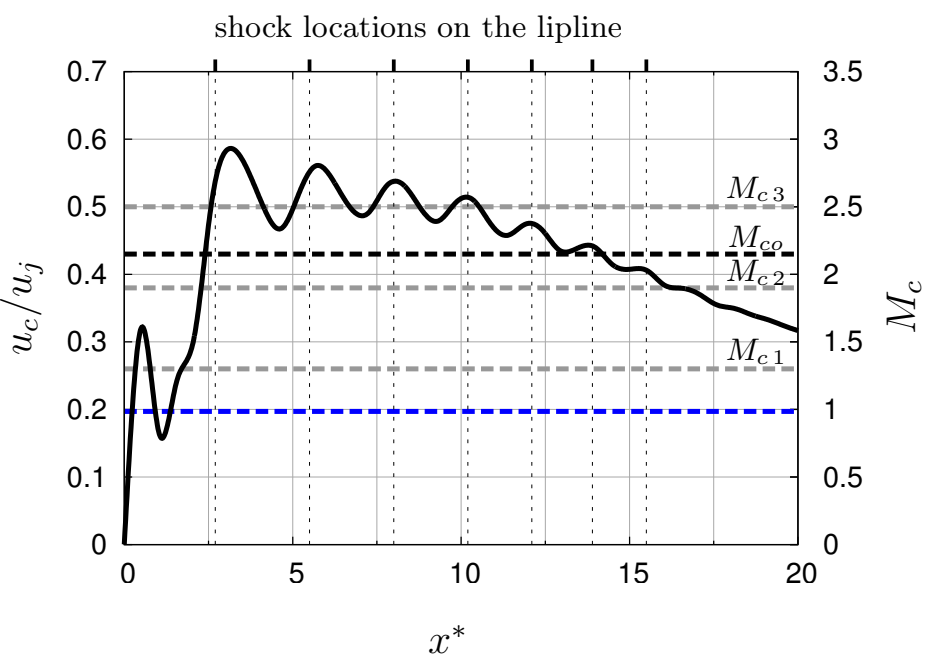

(b)

FIG. 19: estimation of the convection velocity of turbulent structures along the lipline (array A). (19a) normalized double Fourier transform in time and space and $(19 \mathrm{~b})$ convection velocity corresponding to the maximum of the cross-correlation of the axial velocity. - : present numerical data; ---: $u_{c} / u_{j}=0.43$ convection speed; =-1: $M_{c n}$ convection speeds (Eq. 15, 16 and 17); --: $u_{c}=+c_{\infty}$ (supersonic convection threshold).

The initial value is close to 0 due to the previously mentioned flow separation. Three main regions can be deduced from this result. In the early developing shear layer in the range $x^{*}<2$, the most coherent structures generated by the mixing are not fully accelerated yet and only the first instability wave family $\left(M_{c 1}\right)$ seems to be able to radiate Mach waves. It is probably not correct in practice because array A does not properly follow the shear layer in this region due to its deviation (see §III A 1). Downstream in range $2<x^{*}<12$, the convection speed reaches its maximum in the vicinity of the $1^{\text {st }}$ shock $\left(x^{*} \simeq 3\right)$ then oscillates around $M_{c 3}$. Consequently, strong Mach waves are expected to radiate close to the angle $\theta_{M_{c_{3}}}^{\prime}=66^{\circ}$ in this region. The maximum speed at $x^{*}=3$ gives the largest possible radiation angle $\theta_{\max }^{\prime}=70^{\circ}$. Downstream of the end of the potential core for $x^{*}>12$, the convection speed decreases quasi linearly, especially from $M_{c o}$ to $M_{c 1}$. Mach waves can therefore be generated with various radiation angles roughly in range $40^{\circ}<\theta^{\prime}<60^{\circ}$ which is observed in Figure 15 a.

The analyses from the double FFT and the crosscorrelations lead to coherent conclusions. The double FFT provides the most likely convection speed and consequently the Mach waves at the most likely radiation angle $\theta_{M_{c o}}^{\prime}=63^{\circ}$ while the successive cross-correlations indicates that a large variety of Mach waves are possible mainly with radiation angles $\theta_{M_{c 1}}^{\prime}<\theta^{\prime}<\theta_{M_{c 3}}^{\prime}$. Finally, the Mach waves are often associated with the peak directivity but $\theta_{\text {peak }}^{\prime}$ observed in Figure 16 cannot be readily linked to $\theta_{M_{c o}}^{\prime}$ because of a further geometrical bias. The Mach wave generation is indeed not localized in a re- stricted region and the microphones are not far enough from the sources, especially at shallow angles. Consequently, the observation angle transformation is insufficient to get rid of the geometrical bias at shallow angles.

\section{Broadband shock-associated noise}

The shock cells are known to produce noise through an interaction mechanism with convected large turbulent structures. ${ }^{59}$ This noise source has a broadband spectrum, except when a feedback loop with the early instability waves development occurs, inducing a tonal noise known as screech. ${ }^{3}$ The broadband shock-associated noise (BBSAN) is dominant essentially upstream. A model has been proposed by Tam and Tanna ${ }^{59}$ to predict the BBSAN peak frequency according to

$$
S t_{\mathrm{BBSAN}}=\frac{u_{c}}{\overline{L_{\text {shock }}^{*}}\left(1-M_{c} \cos \theta^{\prime}\right) u_{j}}
$$

where the dependency on $\cos \theta^{\prime}$ allows to take into account the Doppler effect, $\overline{L_{\text {shock }}^{*}}$ is the mean shock spacing and $M_{c}=u_{c} / c_{\infty}$ the convective Mach number of the large structures. The mean shock spacing in the Tam and Tanna's model is usually approximated via equation (7) but for the present jet, the shock spacing is different on the centerline and the lipline and get significantly shorter when moving downstream. Given that roughly the $2^{\text {nd }}$ to the $5^{\text {th }}$ shocks are known to be the main contributors to the BBSAN, $\overline{L_{\text {shock }}^{*}}$ is consequently estimated as the 
mean shock spacing between the $1^{\text {st }}$ and the $6^{\text {th }}$ shock in the shear layer, reported in Figure 19b, leading to $\overline{L_{\text {shock }}^{*}}=2.2 \pm 0.6$. The convection Mach number is set equal to $M_{c 3}$. The formula yields $S t_{\mathrm{BBSAN}}=0.15 \pm 0.05$ at $\theta^{\prime}=100.9^{\circ}$ and $S t_{\mathrm{BBSAN}}=0.09 \pm 0.02$ at $\theta^{\prime}=128.6^{\circ}$. These values are in agreement with the peak frequencies noticed on the PSDs at the same angles, respectively in Figures $18 \mathrm{c}$ and $18 \mathrm{~d}$. The uncertainties on $M_{c}$ and $\overline{L_{\text {shock }}^{*}}$ explain the shock-associated noise broadening. When screech noise emerges, its fundamental frequency can be estimated by applying equation (22) at $\theta^{\prime}=180^{\circ}$ yielding $S t_{\mathrm{BBSAN}}=S t_{u p}=0.066 \pm 0.018$. Intense discrete peaks at a frequency $S t_{\text {up }}$ are not observed on the PSDs in the far field, indicating the absence of screech noise as expected for such a hot supersonic jet. ${ }^{2}$

\section{B. Velocity and pressure near field}

\section{Spatio-frequency content}

Azimuthally averaged PSDs of the pressure signal recorded on array $\mathrm{B}$ are plotted as a function of $x^{*}$ in Figure 20. This array is a priori located in a purely acoustic region to characterize the near field wave emission. Regarding the shapes in Figure 20a, the effective cut-off frequency is larger than the global acoustic cutoff $S t_{c}=0.22$ which is due to the fact that the cut-off in the zone Z1 is higher (see §II C 2). The spectrogram in Figure 20b shows various behaviors regarding three different regions. For $x^{*}<4$, low acoustic levels are found excepted a peak at the frequency $S t_{u p}=0.066$ previously highlighted in section IV A 2 as the upstream BBSAN frequency. Since the present jet is not screeching, this peak can simply be attributed to the upstream propagation of the BBSAN. For $4<x^{*}<14$, the spectra are dominated by mid frequencies $S t>S t_{u p}$. For $x^{*}>14$ and downstream, the central frequency decreases and reaches higher levels. This decomposition in three regions is reminiscent of the similar analysis presented in section IV A 1. The Mach waves radiating at largest angles in range $2<x^{*}<12$ on array $\mathrm{A}$ can be associated with mid frequencies in range $4<x^{*}<14$ on array B. The Mach waves radiating at smallest angles in range $x^{*}>12$ on array $\mathrm{A}$ can be associated with low frequencies in range $x^{*}>14$ on array $\mathrm{B}$. Moreover, the BBSAN is mostly generated where the shocks are the strongest and turbulent structures sufficiently developed i.e. roughly $2<x^{*}<16$, at frequencies $S t \geq S t_{u p}$, which is consistent with the spectrogram in the regions $x^{*}<4$ and $4<x^{*}<14$.

A double FFT and cross-correlations of the pressure signals are performed on array B in the same way as for array A. The resulting normalized magnitude of the double FFT is given in Figure 21a. The successive cross-correlations provide the dominant wave phase speed along array B and is drawn in Figure 21b. The phase speed $c_{\varphi}$ is not necessarily equal to $c_{\infty}$ since the waves can propagate in various directions. Assuming the waves are planar fronts having a phase speed $c_{\infty}$ in the propagation direction, the radiation angle can be estimated by $\theta^{\prime \prime}=\arccos \left(c_{\infty} / c_{\varphi}\right)$. Three different behaviors are once again visible and linked to the previously mentioned three jet regions. For $x^{*}<2$, a negative phase speed is found which indicates upstream propagating waves as concluded before. This is corroborated by the double FFT where significant levels are reached, especially at the frequencies $S t \geq S t_{u p}$, along a slope equivalent to a phase speed $c_{\varphi} / c_{\infty}=-1.2$ on the negative wave number side. It means that the waves propagate backward regarding array $\mathrm{B}$ at an estimated angle $\theta_{u p}^{\prime \prime}=145^{\circ}$. Downstream, the phase speed is positive. The peak at $x^{*}=3$ could be attributed to the waves emitted in the vicinity of the $2^{\text {nd }}$ shock, associated with early radiating Mach waves and BBSAN. For $4<x^{*}<14$, the phase speed is rather constant around $c_{\varphi} / c_{\infty}=3.3$ where strong directional Mach waves, generated in the region $2<x^{*}<12$ in the shear layer as mentioned in section IV A 1, are propagating. Downstream for $x^{*}>14$, the phase speed gradually decreases which is associated with Mach waves radiating at smaller and smaller angles. The maximum levels of the double FFT are found for a wide range of frequencies along the slope corresponding to $c_{\varphi} / c_{\infty}=3.3$. The estimated radiation angle is $\theta^{\prime \prime}=72^{\circ}$ which is rather consistent with the larger Mach wave radiation angle $\theta_{\max }^{\prime}=70^{\circ}$ discussed in section IV A 1 . The faint slope corresponding to $c_{\varphi} / c_{\infty}=0.2$, also highlighted on the positive wave number side, is due to slow flow events reaching intermittently array $B$.

\section{Azimuthal content}

The spatio-frequency properties of the Mach wave radiation and the BBSAN in the near field are well assessed at this point. The azimuthal properties are also a matter of interest and are discussed below. An azimuthal mode decomposition is performed on arrays $\mathrm{A}$ and $\mathrm{B}$ at each axial location. It consists in a double FFT in time and azimuth. The results are reported in Figure 22 in terms of energy distribution between modes denoted $m$. The axisymmetric mode corresponds to $m=0$. The highest calculable mode is $m=35$, i.e. the $36^{\text {th }}$ mode, since the numerical arrays include 72 points in the azimuthal direction. The contribution of the first eight modes of the axial velocity on array $\mathrm{A}$ is depicted in Figure 22a. The shock locations in the shear layer are represented with vertical dotted lines. The initial contribution of modes $m \leq 4$ is negligible and the major part of the energy $(\sim 90 \%)$ is equally distributed on higher modes, which are not all drawn. In range $2<x^{*}<14$, modes 1 , 2 and 3 become dominant while mode 0 remains weak even downstream of the end of the potential core as obtained by de Cacqueray et al. ${ }^{34}$ or Liu et al. ${ }^{53}$ The shock cell structure has an important impact on the azimuthal content. The shock locations seems to match with peaks 


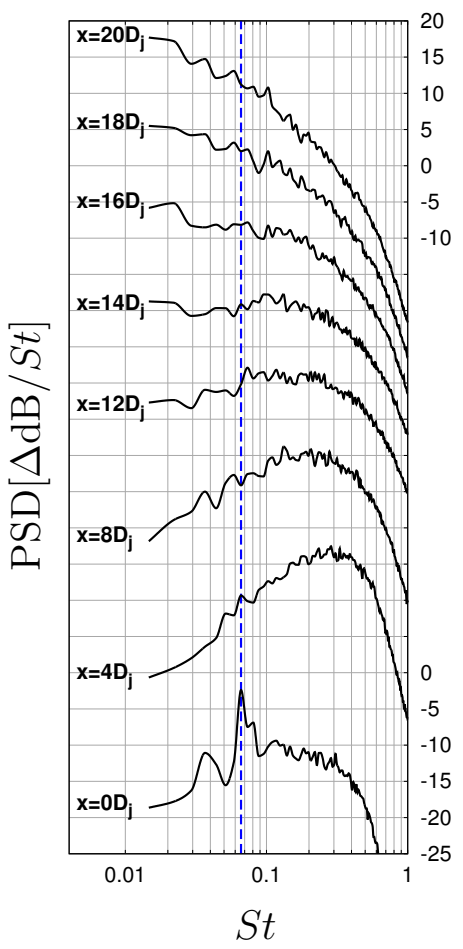

(a)

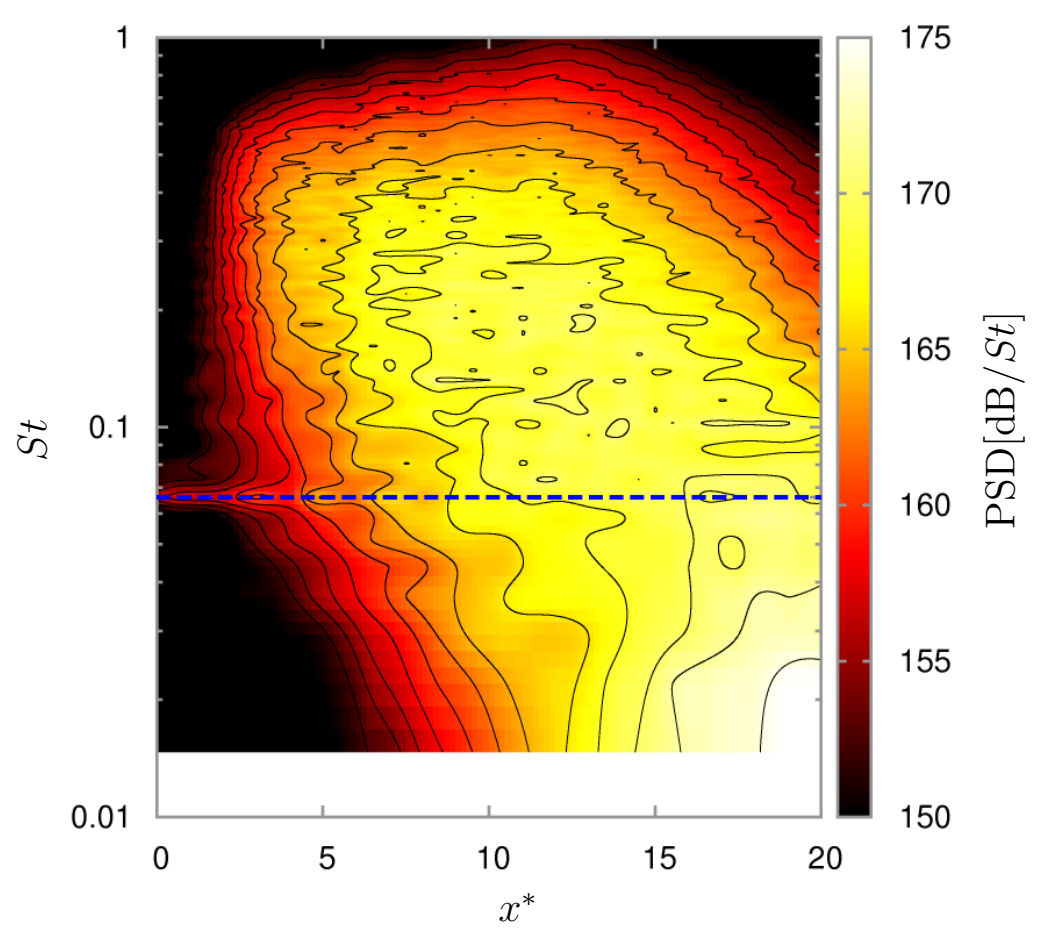

(b)

FIG. 20: PSDs of the pressure measured along array B. (20a) PSDs offset by $10 \mathrm{~dB}$ from others and (20b) spectrogram function of $x^{*}$. —: present numerical data; --.: St $=S t_{u p} ;$ —: PSD iso-lines every $\Delta 2 \mathrm{~dB} / S t$.

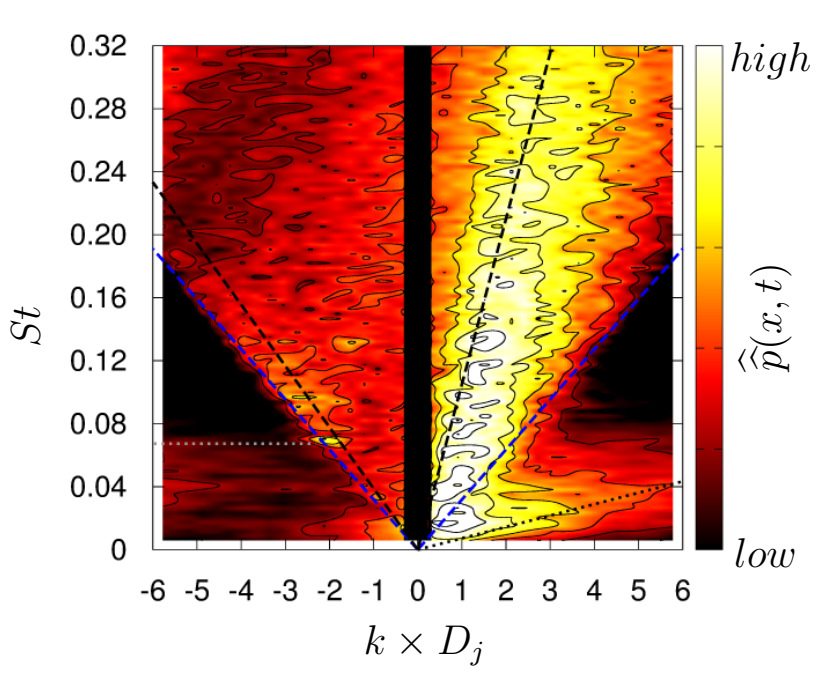

(a)

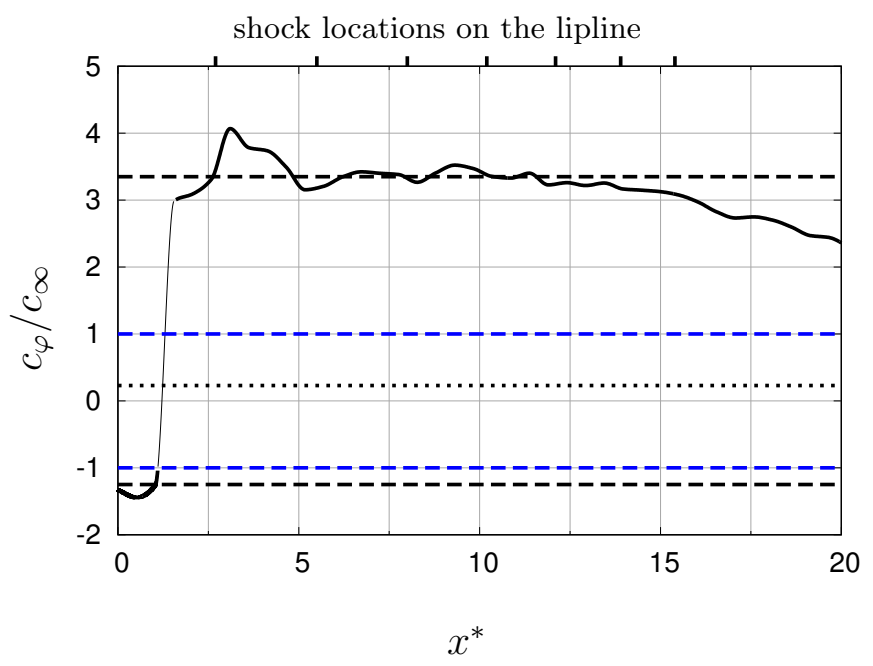

(b)

FIG. 21: characterization of the acoustic wave propagation in the near field. (21a) normalized double Fourier transform in time and space and $(21 \mathrm{~b})$ phase velocity corresponding to the maximum of the cross-correlation of the pressure along array B. - : present numerical data; ---: phase speeds $c_{\varphi} / c_{\infty}=-1.2$ backward and $c_{\varphi} / c_{\infty}=3.3$ forward; $\cdots \cdot$ : slow flow component; $\cdots:$ : St $=S t_{u p} ; \cdots: c_{\varphi}= \pm c_{\infty}$ thresholds. 


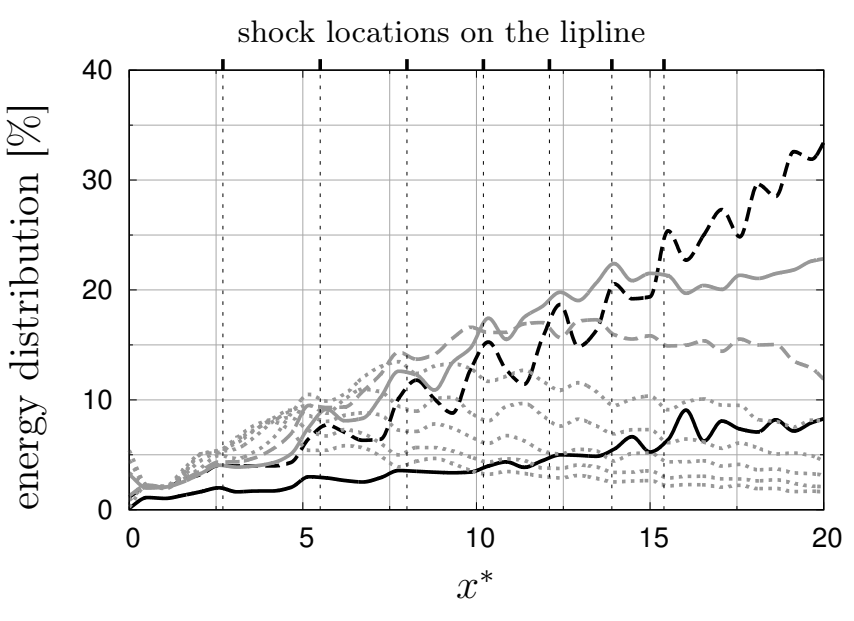

(a)

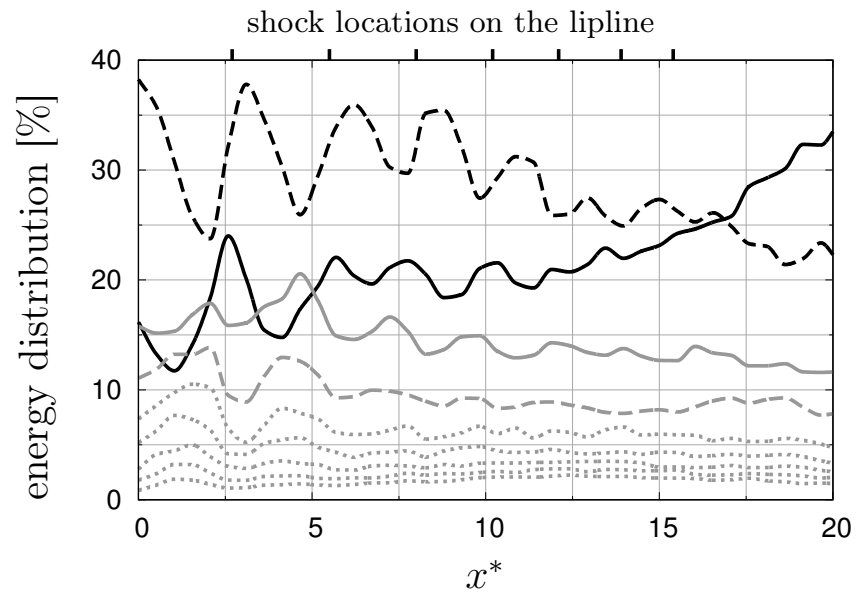

(b)

FIG. 22: energy contribution of the first eight azimuthal modes of (22a) the axial velocity along the lipline (array A) and (22b) the pressure along array B. $m=0 ;-m: m=1 ;=: m=2 ;=m: m=3 ; n: 4 \leq m \leq 8$.

of modes 1 and 2 , and valleys of modes $m \geq 4$. It can be deduced that the shock cell structure favors particular modes which is consistent with observations of many authors studying the BBSAN in axisymmetric free jets, especially screech tones. ${ }^{2,11,53}$ However, this analysis does not permit to definitely conclude on the nature of the modes i.e. flapping (mode B) or helical (mode C) which would require a treatment such as a proper orthogonal decomposition.

The contribution of the first eight modes of the pressure on array B is depicted in Figure 22b. Similar bumped shapes are found for the mode 1 in the range $0<x^{*}<12$ where it is dominant, at the expense of higher modes $m \geq 2$ which exhibit inverse bump patterns. It highlights the strong link between the mode 1 and the noise generation mechanisms in the shear layer in this region. The axisymmetric mode contribution starts to increase for $x^{*}>12$ and becomes predominant downstream for $x^{*}>17$. Bogey and Bailly ${ }^{68}$ have proposed a mechanism consisting in intermittent intrusions of vortical structures inside the end of the potential core. These structures are suddenly accelerated on the jet axis, exciting the acoustic azimuthal mode 0. Bogey and Bailly have suggested that it could be the major noise source radiating downstream at shallow angles in subsonic jets, but a similar mechanism can occur for supersonic jets, causing the dominant mode 0 observed at the downstream extremity of array B.

\section{Pressure far field}

\section{Spatio-frequency content}

The acoustic far field has been succinctly presented in section III B 2 and section III B 3 in terms of OASPL and PSDs at four observation angles for validation purposes. This section aims at providing extended results and analysis. The PSDs and the corresponding spectrogram of the pressure signal at the far field microphones are drawn in Figure 23. PSDs peak frequencies are roughly distributed from $S t=0.02$ at shallowest angle up to $S t=0.2$ on the sideline. The Mach wave radiation corresponds to the broadband high level region for $\theta^{\prime}<70^{\circ}$ on the spectrogram. The BBSAN corresponds to the thinner band extending upstream for $\theta^{\prime}>90^{\circ}$. The BBSAN peak frequency function of the observation angle $\theta^{\prime}$ is plotted as blue dashed lines according to equation (22). It coincides with PSDs peaks from $\theta^{\prime} \gtrsim 90^{\circ}$ and tends to $S t_{\text {BBSAN }}=S t_{u p}$ upstream as expected. Such far field spectrogram patterns are similar to what has been obtained experimentally for analogous jets, by Schlinker et al. ${ }^{69}$ for example.

The Mach wave radiation and the BBSAN appear to be both easily identifiable in the far field regarding the PSDs peak frequency variation function of the observation angle. The peak frequency field depicted in Figure $24 \mathrm{~b}$ is obtained by extracting the peak of the pressure PSD at each point of the mesh intersecting a 2D plane. It enables to highlight more clearly main acoustic regions. In addition, the root mean square pressure field is given in Figure 24a. The patterns appear to be roughly centered on the end of the potential core which justified the use of the transformed angle system. For angles $\theta^{\prime}<70^{\circ}$ which corresponds to all possible Mach wave radiation angles $\left(\theta^{\prime}<\theta_{\max }^{\prime}\right)$, the noise is dominated by frequencies approximately in range $0.01<S t<0.1$. The very low peak frequencies observed at shallowest angles in the zone $\mathrm{Z} 1$ are due to slow flow events. At larger angles and especially close to the sideline at $\theta^{\prime} \simeq 90^{\circ}$, the noise is dominated by the BBSAN in mid frequencies up to the cut-off frequency $S t_{c}=0.22$. The peak 


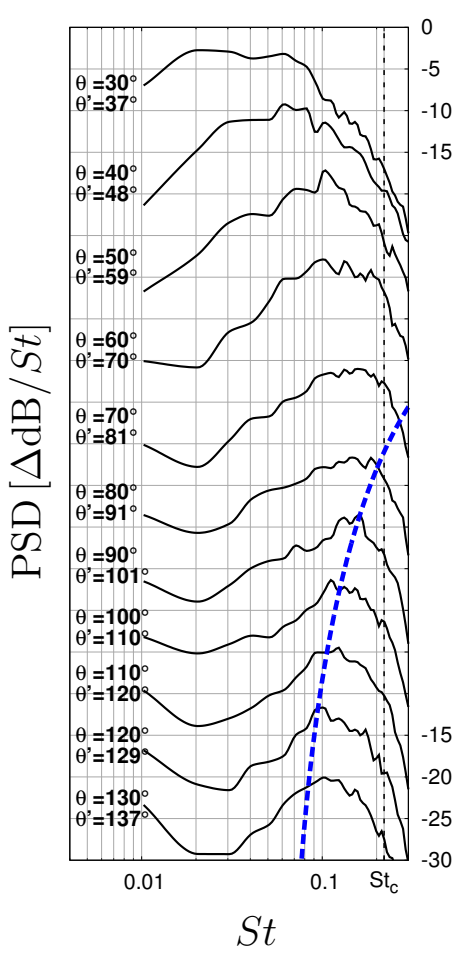

(a)

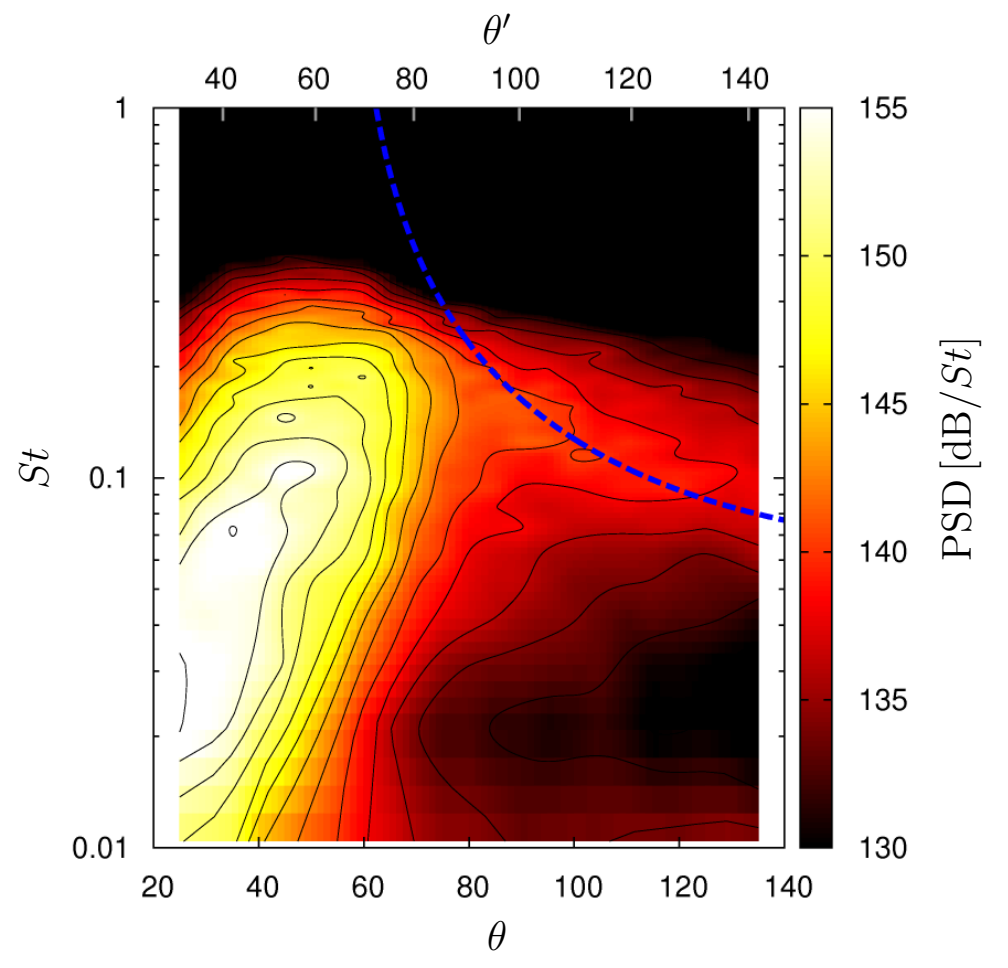

(b)

FIG. 23: PSDs of the pressure measured at far field microphone locations with (23a) PSDs offset by $7 \mathrm{~dB}$ from others and (23b) spectrogram function of $\theta$. - : present numerical data; -.-: $S t=S t_{\mathrm{BBSAN}}\left(\theta^{\prime}\right)$; —: PSD iso-lines every $\Delta 2 \mathrm{~dB} / S t$.

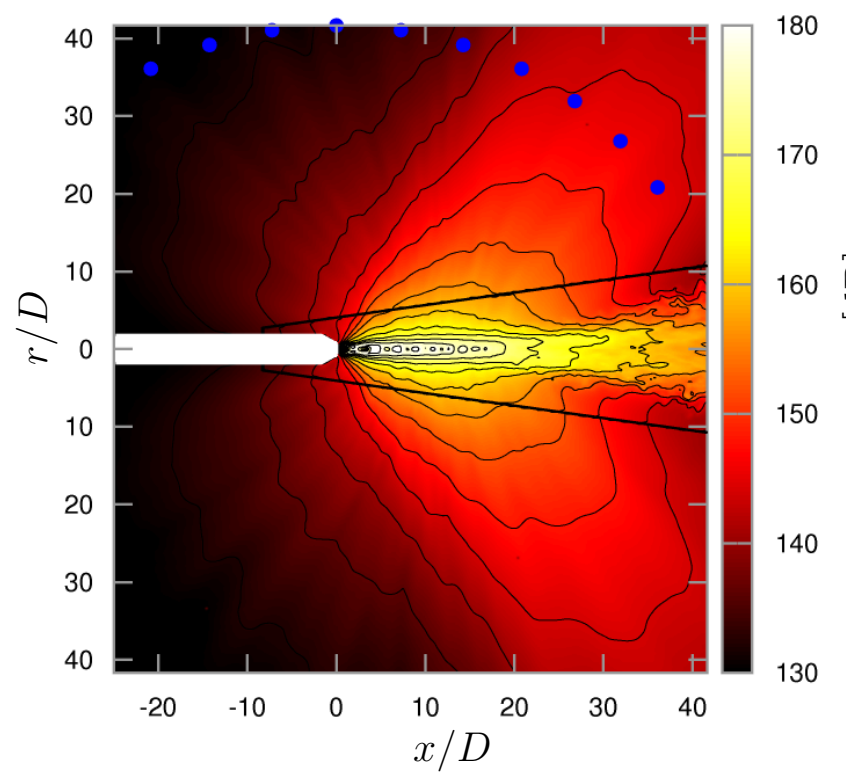

(a)

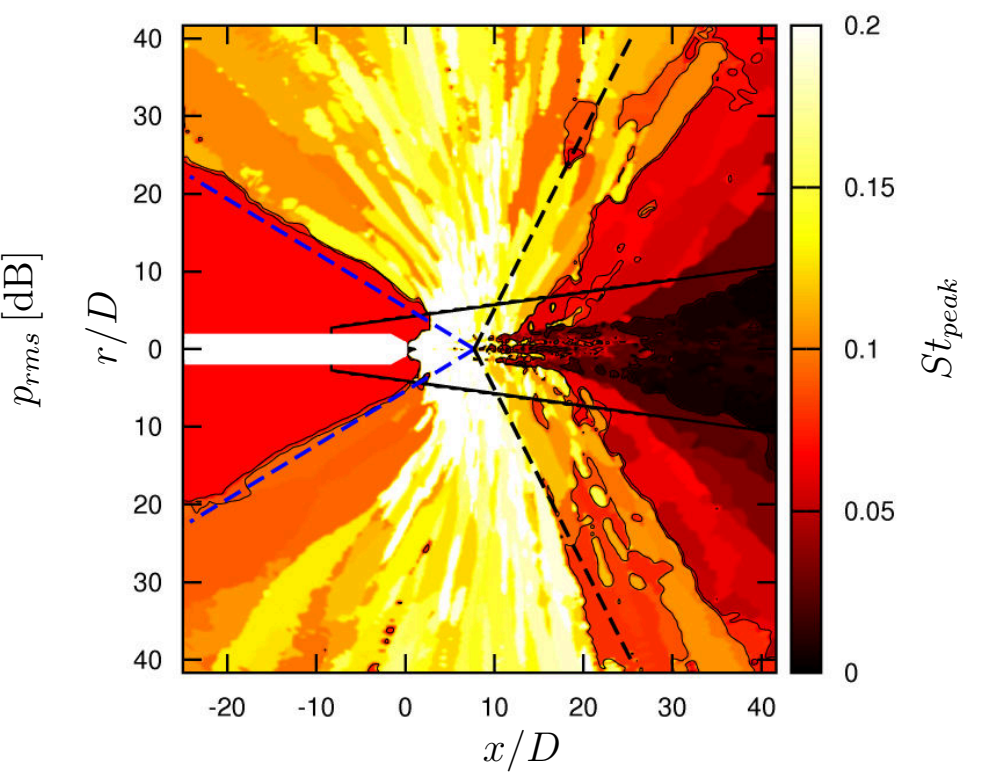

(b)

FIG. 24: (24a) pressure RMS level field and (24b) spectrum central frequency field. ๑: far field microphones;

—: iso- $p_{r m s}$ and iso-frequencies $S t=0.02, S t=S t_{u p}$ and $S t=0.09 ;-$ : coupling interface; $\cdots: \theta^{\prime}=\theta_{\text {max }}^{\prime}=70^{\circ}$;

$$
\text { -.-: } \theta^{\prime}=\theta_{u p}^{\prime \prime}=145^{\circ} \text {. }
$$




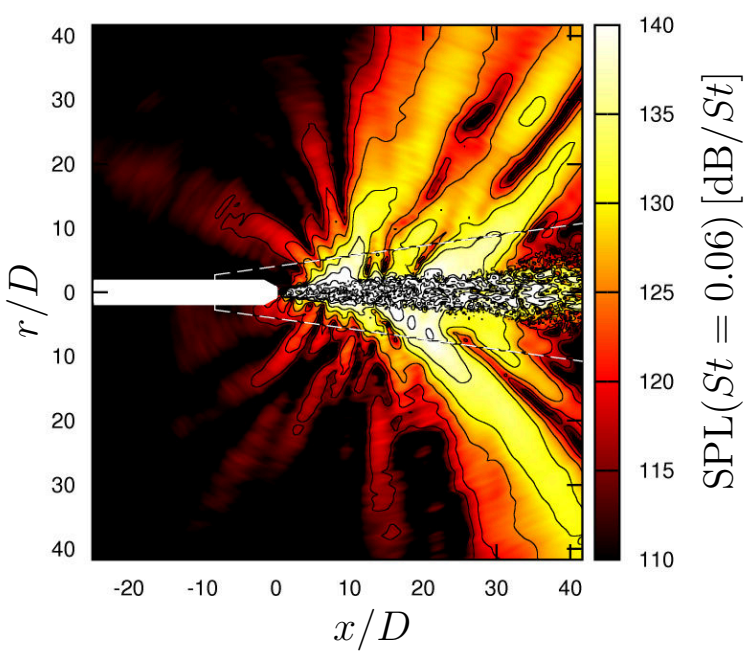

(a)

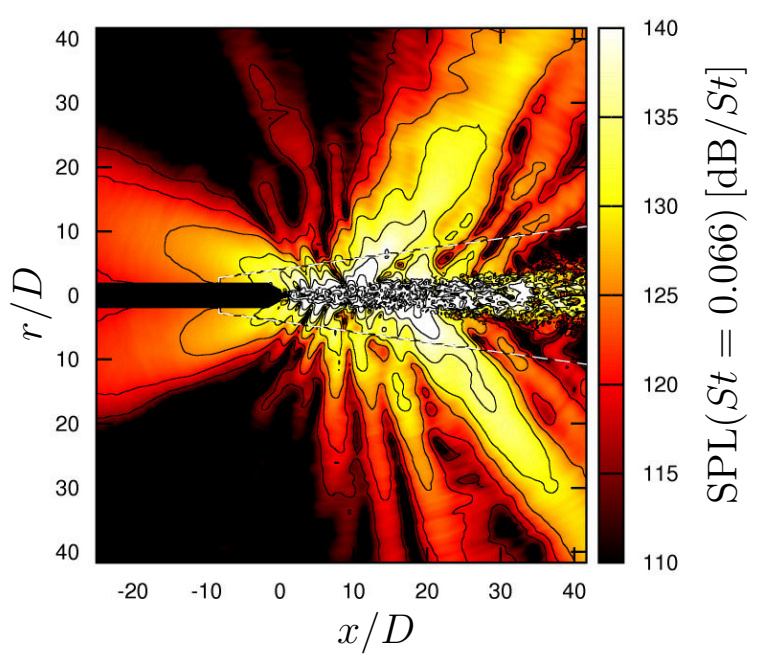

(c)

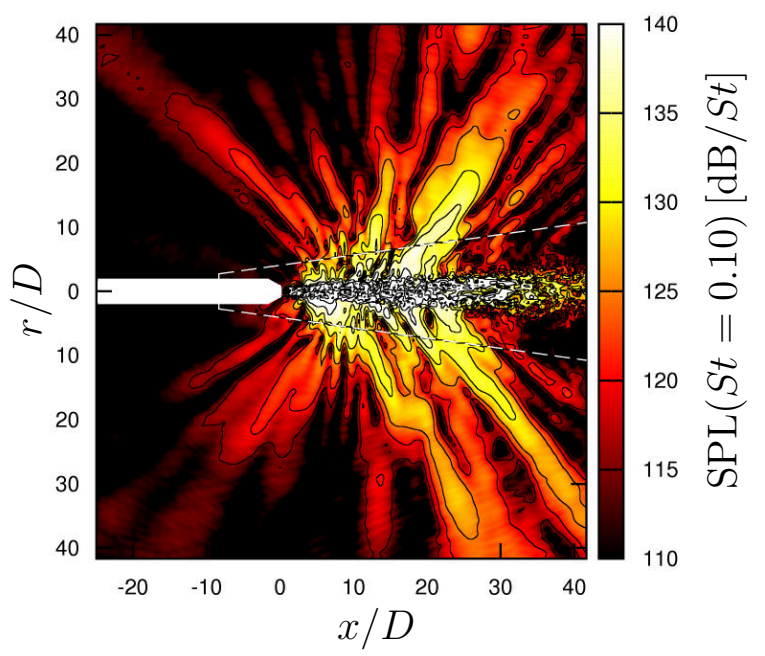

(e)

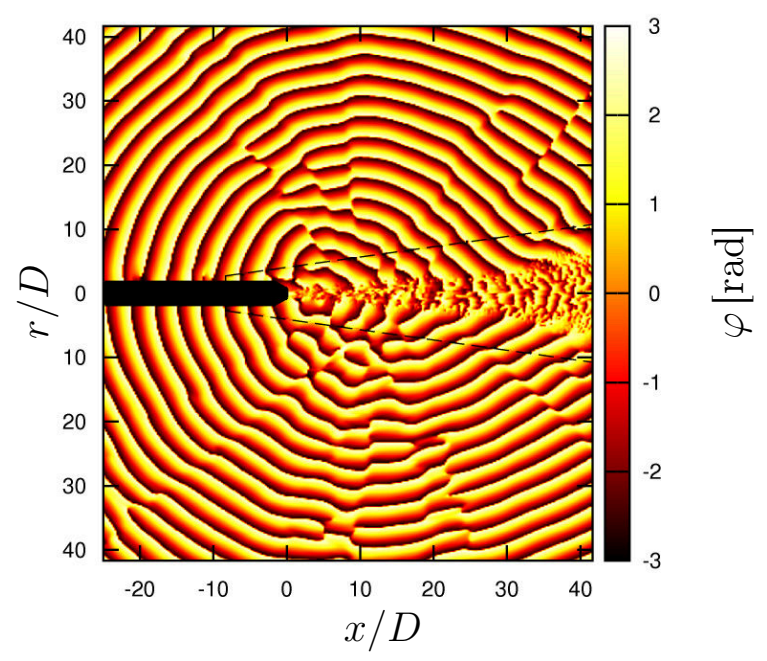

(b)

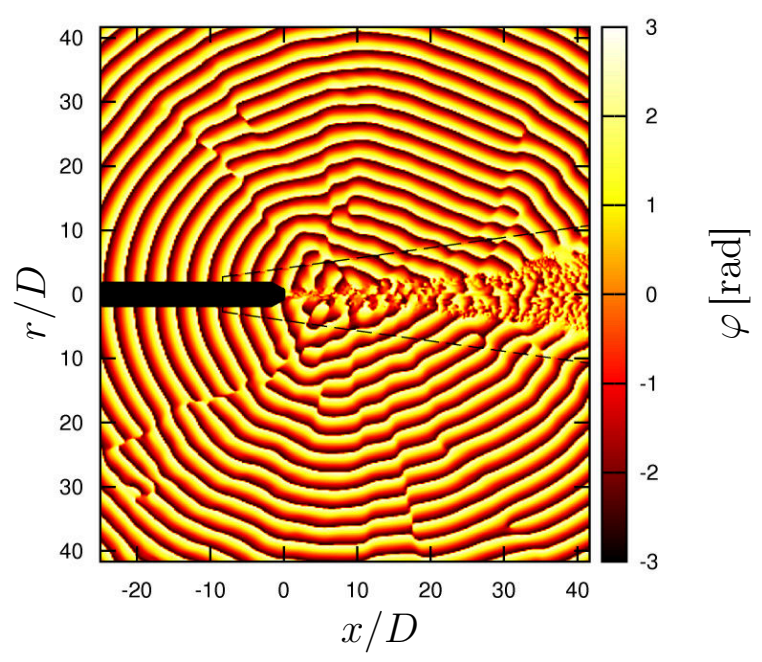

(d)

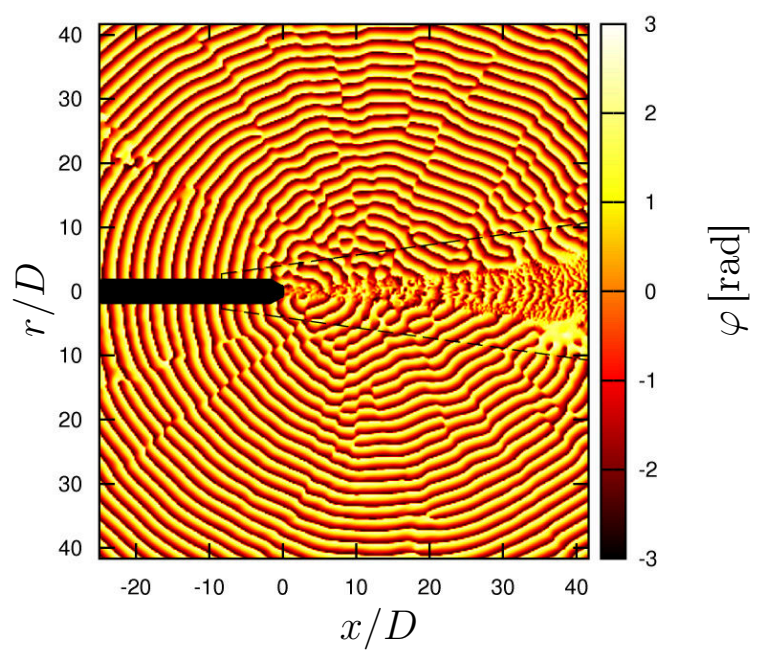

(f)

FIG. 25: pressure RMS level fields and phase fields at three particular frequencies, $(25 \mathrm{a} / 25 \mathrm{~b}) S t=0.060$, $(25 \mathrm{c} / 25 \mathrm{~d}) S t=S t_{u p}=0.066$ and $(25 \mathrm{e} / 25 \mathrm{f}) S t=0.100$. — : iso-lines; --- : coupling interface. 
frequency distribution is strongly inhomogeneous probably because of multiple source locations and interferences. More upstream, the BBSAN peak frequencies decrease due to the Doppler effect. From $\theta^{\prime} \simeq 145^{\circ}$, the distribution become strongly homogeneous at a peak frequency $S t=S t_{u p}$. It cannot be fully explained by the BBSAN model discussed in section IV A 2 since equation (22) yields $S t_{\mathrm{BBSAN}}\left(\theta^{\prime}=145\right)=0.077 \neq S t_{u p}$. Tam $^{2}$ or Bailly and Fujii ${ }^{3}$ have suggested that the instability wave interaction theory, from which equation (22) is derived, also predicts instability waves traveling in the upstream direction. These waves could generate Mach waves if their phase speeds $c_{\varphi}$ are supersonic, i.e. if

$$
\begin{aligned}
c_{\varphi} & <-c_{\infty} \\
\frac{\omega}{\alpha-k_{\text {shock }}} & <-c_{\infty}
\end{aligned}
$$

where $\omega=2 \pi f, \alpha \simeq \omega / u_{c}$ and $k_{\text {shock }}=2 \pi / D_{j} \overline{L_{\text {shock }}^{*}}$. Considering $\omega=2 \pi S t_{u p} u_{j} / D_{j}, u_{c}=0.5 u_{j}\left(M_{c 3}\right)$ and $\overline{L_{\text {shock }}^{*}}=2.2$, this requirement is presently met and Mach wave are consequently expected to radiate upstream at a very large angle $\theta^{\prime}>160^{\circ}$ according to equation (18). It could explain the upstream dominant radiation at the frequency $S t_{u p}$ and angles $\theta^{\prime}>160^{\circ}$. However, these waves are also dominant in the range $145<\theta^{\prime} \leq 160^{\circ}$ which is corroborated by the angle $\theta_{u p}^{\prime \prime}=145^{\circ}$ found in section IV B 1 analyzing the upstream radiation along array $B$. It can be related to the axial variation of $u_{c}$ and $L_{\text {shock }}^{*}$ and the associated uncertainties. According to Figure 20a, this Mach wave-like upstream BBSAN, denoted MW-BBSAN in the following, has a lower intensity of about $15 \mathrm{~dB}$ compared to the standard downstream Mach waves.

The SPL and phase fields for three particular frequencies, including $S t_{u p}$, are shown in Figure 25. The hypothesis of an upstream Mach wave radiation at the discrete frequency $S t_{u p}$ (MW-BBSAN) is corroborated. Significant acoustic levels are indeed found upstream in Figure $25 \mathrm{c}$ and the radiation is strongly directional around $\theta^{\prime}=155^{\circ}$. Such patterns are not recovered for other frequencies in Figures 25a $(S t=0.06)$ and $25 \mathrm{e}(S t=0.10)$. Large bulbs corresponding to the standard Mach wave radiation are only found downstream. The multiple thinner bulbs mainly radiating in the sideline and upstream directions correspond to the BBSAN. The phase field in Figure 25d clearly highlights that the upstream radiation at $S t_{u p}$ is associated with the shock cell periodicity in the near field. The involved wave fronts are generated in opposition of phase in the cut plane, as also observed in Figures 25b and 25f. This observation is consistent with the azimuthal dominant mode 1 in the near field close to the strongest shocks (see §IV B 2, Figure 22b) and in the far field in the upstream direction (see §IV C 2, Figure 26a).

\section{Azimuthal content}

The contribution of the pressure azimuthal modes in the far field can be readily related to the acoustic near field. Figure 26 shows three angular regions with specific behaviors. Upstream at angles $\theta^{\prime}>90$, the noise is dominated by the BBSAN and azimuthal mode contributions are rather constant as a function of the angle. More than one third of the energy is included in mode 1 which can be associated with the mode 1 excited by the shock cell structure in the shear layer (Figure 22a) and the strongly dominant mode 1 in the acoustic near field (Figure 22b). The range $70^{\circ}<\theta^{\prime}<90^{\circ}$, roughly the sideline direction, is a transition region between Mach waves and BBSAN. The contributions of modes 0 and 1 drop in favor of higher modes. This region can be associated with the inhomogeneous high peak frequencies in Figure 24 discussed in section IV C 1 . Downstream at angles $\theta^{\prime}<70$, the noise is dominated by Mach waves essentially at mode 0 . Mach waves radiating at the highest possible angles $60^{\circ}<\theta^{\prime}<70^{\circ}$ also contain an important mode 1 component. These waves are generated in the shear layer where the convection speed is maximum i.e. before the end of the potential core (see Figure 19b). They are therefore probably highly perturbed by the shock cell structure. At shallower angles, the contribution of the axisymmetric mode strongly increases due to the intermittent intrusion of vortical structures inside the end of the potential core as discussed in section IV B 2 .

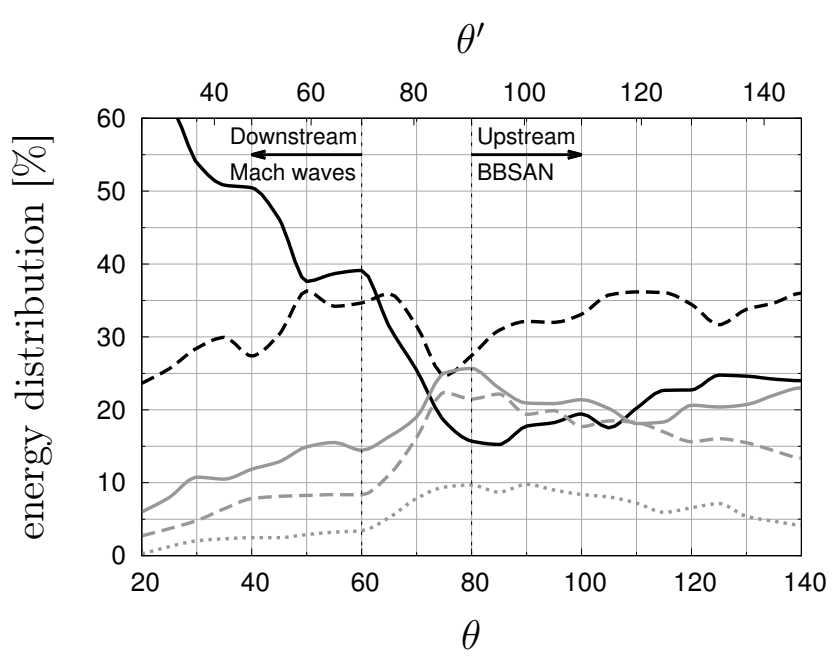

(a)

FIG. 26: energy contribution of the first five azimuthal modes of the pressure at far field microphone locations

function of $\theta$ and $\theta^{\prime} . \longrightarrow: m=0 ; \boldsymbol{- m}: m=1$; : $m=2 ;= \pm: m=3 ; " \|: m=4$. 


\section{Nonlinear propagation}

\section{Near field mechanism and cumulative effects}

The acoustic nonlinear propagation is a key point of this study. Noise from hot supersonic rocket plume involves nonlinear effects, ${ }^{70}$ often associated with the crackle noise. ${ }^{71}$ However, the relative importance of local mechanisms in the near field and cumulative effects during propagation in the far field is still unclear. On the one hand, harsh flow events generating steep acoustic waveform directly emerging from the shear layer have been found to occur. This mechanism is known to be closely linked to the Mach wave radiation and is originally considered as the primary source of crackle. It has been observed experimentally ${ }^{40}$ and reproduced numerically for supersonic jets by Nichols et $a .^{6}$ on a military-style configuration and more recently by Pineau et $a l . .^{72}$ using temporally-developing jets, among the others. On the other hand, cumulative nonlinearities have been highlighted in the acoustic far field of supersonic jets by several authors, both experimentally ${ }^{5,64}$ and numerically. ${ }^{19,44}$ Such effects mainly consist in compression waves gradually steepening which is equivalent to a distortion of the probability density function of the signal, or in the frequency domain to an energy transfer from the peak to higher frequencies. Petitjean et al. ${ }^{5}$ has found that $M_{c}>1$ was a necessary condition for the onset of this kind of nonlinearities which is also a critical parameter for the Mach wave occurrence. As suggested in Figure 19b, this requirement is presently fulfilled on the lipline over a wide range of axial locations.

\section{Metrics}

The acoustic near and far fields are analyzed by focusing on the expected nonlinear effects. Specific metrics are introduced for that purpose, including the Goldberg number $\Gamma$, the skewness $S$ (Eq. (8)), the kurtosis $K$ (Eq. (9)) and the wave steepening factors WSF. The Goldberg number is defined by

$$
\Gamma=\frac{\beta \omega p_{r m s} \lambda}{\rho_{\infty} c_{\infty}^{3} \alpha(\omega)}
$$

where $\beta=(1+\gamma) / 2$ is the nonlinearity coefficient and $\alpha(\omega)$ the dimensionless atmospheric damping function of the frequency, here estimated according to the standard ISO-9613. ${ }^{51}$ It is assumed that $\Gamma<1$ reflects a linear propagation and $\Gamma \gg 1$ a nonlinear propagation. ${ }^{7}$ The reference values for a Gaussian signal are $S=0$ and $K=3$. Note that compared to the raw pressure metrics $S(p)$ and $K(p)$, the pressure derivative metrics $S(\dot{p})$ and $K(\dot{p})$ are often considered as more sensitive and relevant to study nonlinear effects. ${ }^{5,7,43}$ In both cases, $S$ and $K$ increase when the nonlinear effects are predominant over the atmospheric viscous damping. The WSF is defined as the modulus of the average negative slope divided by the average positive slope of the pressure waveform. ${ }^{7,73}$ The reference value WSF $=1$ corresponds to a pure harmonic waveform and WSF $=0$ to a perfect sawtooth waveform. It decreases when the nonlinear effects are predominant over the atmospheric viscous damping. It can also be larger than 1 and theoretically tends to infinity in case of discontinuous pressure drops. The evolution of these metrics for nonlinearly propagating waves have been studied, in particular by Reichman et al. ${ }^{43}(S(\dot{p}))$ and Muhlestein et al. ${ }^{73}$ (WSF), and experienced in many studies dealing with jet noise issues at various distances. Mora et $a l .{ }^{8}$ have found increasing values of pressure derivative skewness and kurtosis up to $S(\dot{p})>0.8$ and $K(\dot{p})>4$ at a distance of about $25 D$ from a $M>1.5$ hot jet. Baars et al. ${ }^{7,71}$ used the pressure derivative skewness and the WSF to identify cumulative nonlinearities out to $140 D$ from a $M=3$ cold jet in the peak noise direction. Schlinker et al. ${ }^{69}$ have measured raw pressure skewness reaching values greater than 0.5 at an angle $\theta=50^{\circ}$ out to $300 D$ from a $M>1.5$ hot crackling jet.

The metrics mentioned above are plotted in Figure 27 in a cut plane. The far field microphones are marked as blue dots in Figure 27a for analysis convenience. The obtained skewness and kurtosis ranges are consistent with the values found by other authors at similar distances. The metrics based on the pressure time derivative clearly highlight the downstream Mach wave generation and radiation regions where nonlinear propagation effects are the most expected due to high acoustic levels. The metrics based on the raw pressure do not lead to such a result. All metrics indicate that strong nonlinearities also occur in the near field close to the shear layer. In particular, high excesses of $S(\dot{p}), K(\dot{p})$ and values of WSF larger than 1 are noticeable at $x \simeq 25 D$, suggesting sharp pressure raises or drops. A similar behavior have been found for crackling jets and it adds credit to a crackle generation mechanism located in the shear layer. On the other hand, most of the metrics are found to be still significantly varying in the far field out to $40 \mathrm{D}$, especially WSF. It decreases to less than 0.6 in several locations associated with high levels of $S(\dot{p})$ and $K(\dot{p})$ which indicates that compression waves are still steepening due to the nonlinear propagation. It is consistent with the Goldberg number field which shows typical nonlinear values greater than 100 within two lobes extending in the far field in the peak direction.

\section{Method relevancy}

These metrics suggest that nonlinear effects can occur in both the near field and the far field. One could assume that far field nonlinear effects are negligible compared to near field events. This issue is handled by performing a linear Ffowcs Williams \& Hawkings extrapolation from a cylindrical integration surface at a radius $r_{F W H}=11.2 \mathrm{D}$ which is fully included in the Euler domain. The resulting 


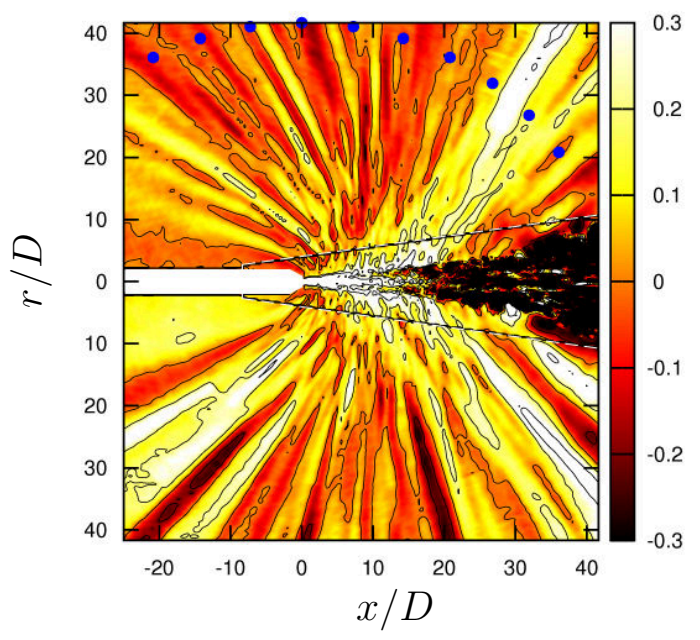

(a)

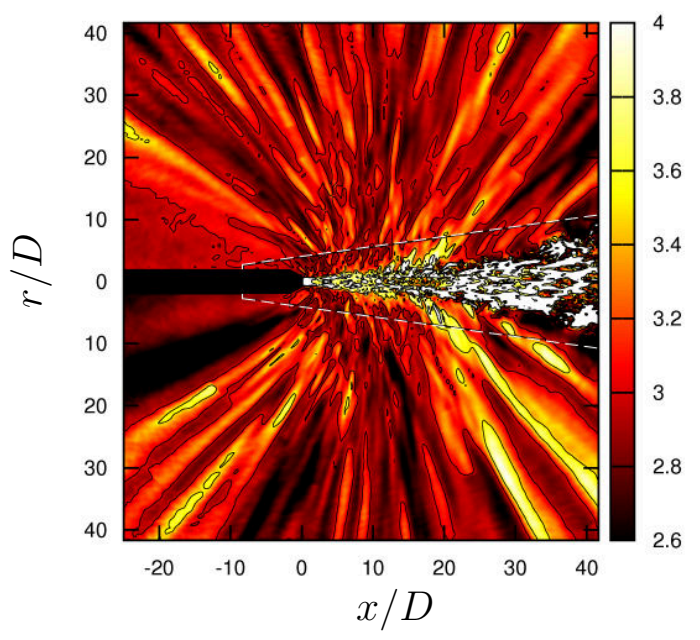

(c)

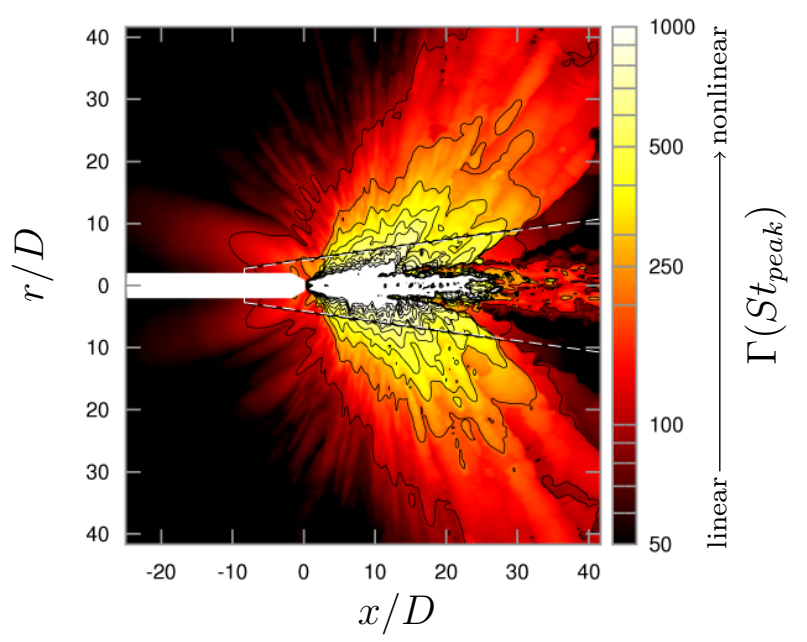

(e)

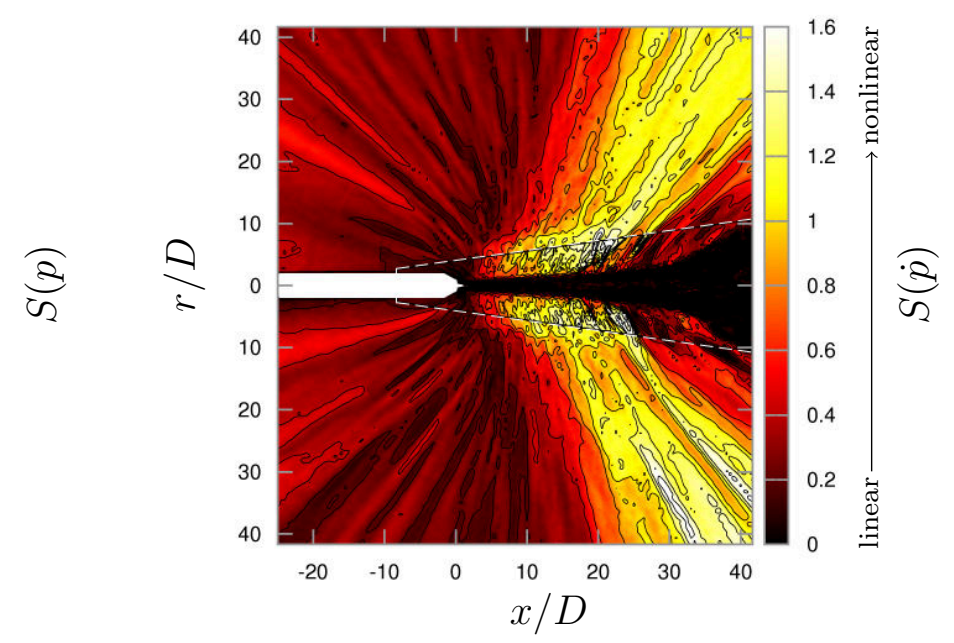

(b)

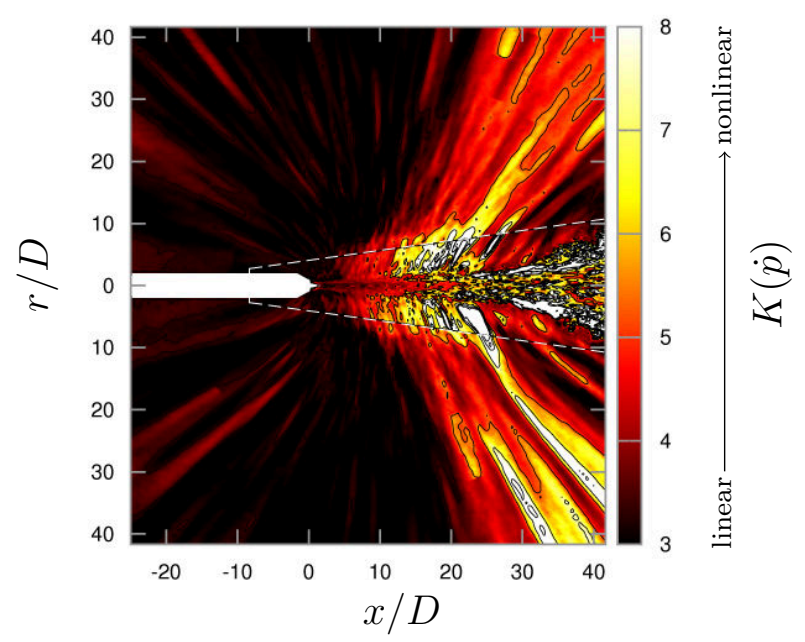

(d)

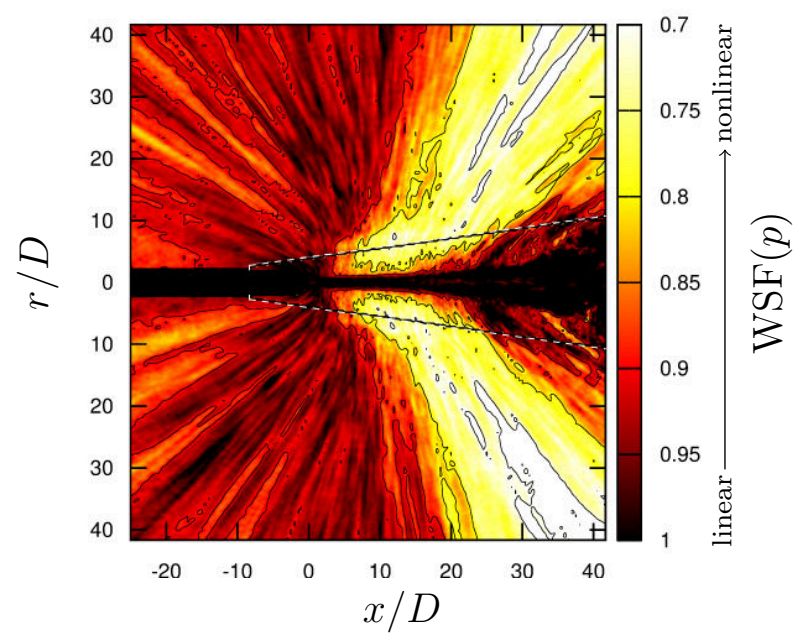

(f)

FIG. 27: nonlinearity metric maps including (27a) raw pressure skewness, (27b) pressure derivative skewness, (27c) raw pressure kurtosis, (27d) pressure derivative kurtosis, (27e) Goldberg number at the peak frequency and (27f) wave steepening factor. @: far field microphones; —-: iso-lines; ---: coupling interface. 


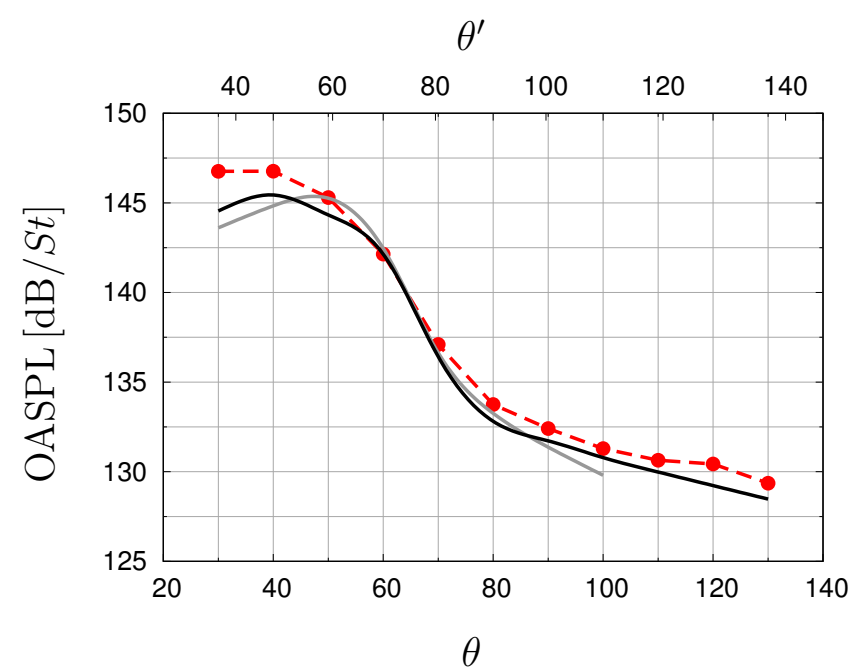

FIG. 28: OASPL at the far field microphones computed by integrating the PSDs over the range $0.01 \leq S t \leq 0.22$. --O- : : experimental data; - $\mathbf{2}$ NS-Euler computation; —: NS-Euler-FWH computation.

acoustic levels at the far field microphones for $\theta \leq 100^{\circ}$ are depicted in Figure 28. The linear computation does not overestimate the levels, which is counter-intuitive regarding the Euler/FWH comparison based on a previous flow solution ${ }^{44}$ in Figure 16. It could indicate that most of the nonlinear effects are included inside the cylinder of radius $r_{F W H}$.

However, the overall acoustic levels alone are definitely not conclusive about the method relevancy. The azimuthally averaged statistics of the pressure signal at two key locations in the far field are given in Table III. The numerical data from the far field microphones at $\theta=40^{\circ}$ and $\theta=90^{\circ}$ allows the comparison with the experimental data. As noted previously, the acoustic levels are similar but significant differences are found for the others metrics (peak Strouhal, $S(\dot{p}), K(\dot{s})$ and WSF). The Navier-Stokes-Euler computation better agrees with the experimental data at the two angles. In particular, significantly higher values of $S(\dot{p})$ and lower values of WSF are found as expected in case of a nonlinear propagation. An extended analysis is proposed with Figure 29. The derivative skewness, kurtosis and the steepening factor calculated both from the experimental and the numerical signals are plotted against the observation angle. The Navier-Stokes-Euler computation provides consistent results with respect to the experiment and clearly demonstrates its ability to take into account nonlinear effects with peak values around the peak direction $\theta^{\prime}=48^{\circ}$ - Such trends are not well recovered by the Ffowcs Williams \& Hawkings approach.

Furthermore, the metric $K(\dot{s})$ presents the higher measurement dynamic but also the larger discrepancies between the experiment and the Navier-Stokes-Euler com- putation, and the lower discrepancies between the Euler and the Ffowcs Williams \& Hawkings computations. The metrics $S(\dot{p})$ and WSF appear to be more robust in quantifying cumulative nonlinearities. $S(\dot{p})$ typically remains below 1.5 in the Navier-Stokes-Euler computation at a distance of $40 D$ from the sources while the experimental data shows a peak $S(\dot{p})>1.75$. Reichman et $a l .{ }^{43}$ have associated shocked waveform appearance with values greater than 1.5 which suggests that shocks exist in experiments but are not properly captured in the computation. It highlights a major limitation of the Navier-Stokes-Euler computation. The cut-off frequency $S t_{c}=0.22$ seems too low to fully reproduce the nonlinear phenomena.

\section{CONCLUSION}

A numerical methodology including a two-way NavierStokes - Euler coupling, a geometrical turbulence tripping and a high-order nonlinear acoustic solver is described and applied to the simulation of a hot $M=3.1$ supersonic jet. The jet parameters correspond to an experiment conducted at the MARTEL facility which aims at investigating the acoustic field during the launcher liftoff. The turbulence tripping method using a small step in the nozzle convergent associated with an important effort on the mesh refinement leads to a major improvement of the flow solution compared to previous works. This approach enables to get velocity fluctuation levels greater than $2 \%$ in the nozzle divergent. A highly disturbed exit condition is thereby obtained at the nozzle lips.

The aerodynamic near field and the acoustic far field fairly agree with the available experimental data. In particular, the shear layer development shows proper features in terms of spread rate, velocity fluctuation levels and turbulent spectra. The far field acoustic levels are recovered within a $1 \mathrm{~dB}$ error at most of observation angles and the spectra are well predicted in all directions despite a slight underestimation at shallowest angles. Based on these results, an extended physical analysis of the radiated noise is performed. The acoustic near and far fields are consistent with the noise generation mechanisms described and modeled in the literature. The noise is generated via two main mechanisms, the Mach wave radiation essentially downstream and the broadband shockassociated noise essentially upstream.

The Mach wave radiation angles are related to the convection speed of large turbulent structures. In the range $2<x^{*}<12$ along the lipline where $x^{*}=x / D_{j}$, this convection speed is maximum leading to Mach wave emission expected at angles $60^{\circ}<\theta^{\prime}<70^{\circ}$. The resulting noise is broadband centered on mid frequencies $S t \simeq 0.1$ and contains an important $m=1$ azimuthal component attributed to the velocity mode 1 in the shear layer. Downstream of the end of the potential core, the convection velocity in the shear layer decreases, leading to Mach waves radiating at angles $40^{\circ}<\theta^{\prime}<60^{\circ}$. An additional mecha- 
TABLE III: azimuthally averaged statistics of the pressure signal from the NS-Euler-FWH computation, NS-Euler computation and the experiment at the far field microphones $\theta=90^{\circ}$ and $40^{\circ}$.

\begin{tabular}{llllllllll}
\hline \hline$\theta$ & $\theta^{\prime}$ & method & $p_{r m s}[\mathrm{~dB}]$ & $S t_{\text {peak }}$ & $S(p)$ & $S(\dot{p})$ & $K(p)$ & $K(\dot{p})$ & WSF \\
\hline \multirow{2}{*}{$90^{\circ}$} & \multirow{2}{*}{$101^{\circ}$} & NS-Euler-FWH & 131.4 & 0.258 & +0.09 & -0.04 & 2.98 & 3.91 & 1.00 \\
& & NS-Euler & 131.7 & 0.154 & -0.03 & +0.25 & 2.96 & 2.90 & 0.94 \\
& Experimental & 132.5 & 0.176 & +0.29 & +0.45 & 3.24 & 3.64 & 0.89 \\
\hline \multirow{2}{*}{$40^{\circ}$} & \multirow{2}{*}{$48^{\circ}$} & NS-Euler-FWH & 144.8 & 0.064 & -0.05 & -0.04 & 2.59 & 4.06 & 1.00 \\
& & NS-Euler & 145.5 & 0.066 & +0.09 & +1.21 & 3.04 & 5.50 & 0.73 \\
& & Experimental & 146.8 & 0.072 & +0.60 & +1.79 & 3.72 & 9.79 & 0.68 \\
\hline \hline
\end{tabular}

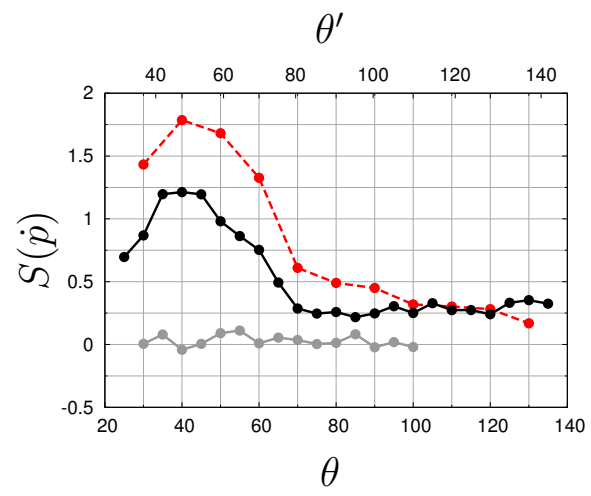

(a)

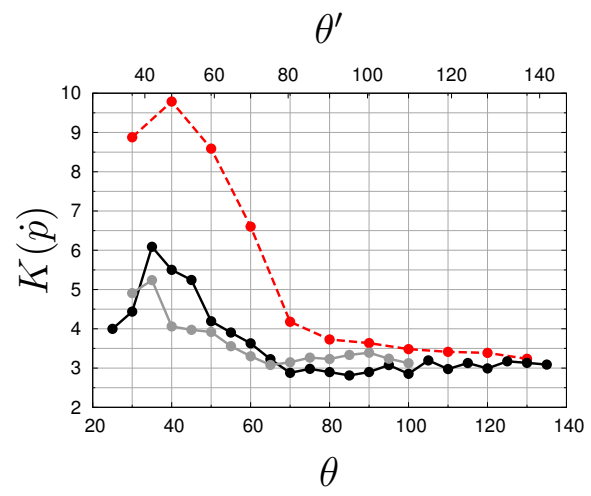

(b)

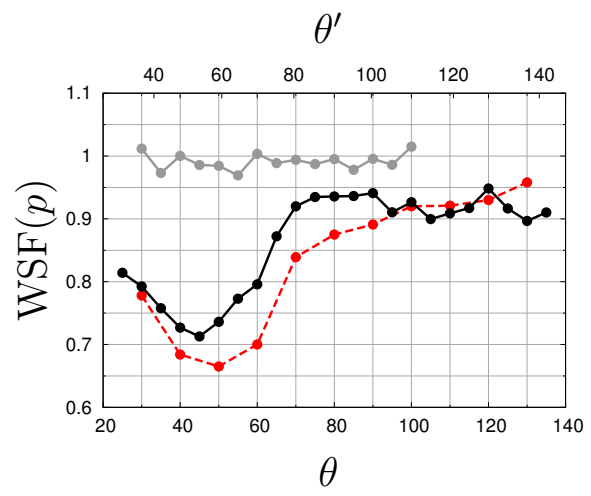

(c)

FIG. 29: azimuthally averaged $S(\dot{p}), K(\dot{s})$ and WSF metrics function of $\theta$ and $\theta^{\prime}$. ---- : experimental data; $\multimap-$ : NS-Euler computation; ——: NS-Euler-FWH conputation.

nism, consisting in the intermittent intrusions of vortical structures inside the end of the potential core and convected on the centerline, can explain the low frequency noise strongly dominated by the azimuthal mode 0 at shallower angles. From these conclusions can also be deduced that the noise sources associated with large turbulent structures are broadly axially distributed.

Close to the sideline direction for angles $70^{\circ}<\theta^{\prime}<90^{\circ}$, the main noise source is not well defined. This angle range is considered as a transition region. The noise is broadband centered on mid frequencies $S t>0.1$ with an important contribution of azimuthal modes $1 \leq m \leq 3$. In the upstream direction for angles $90^{\circ}<\theta^{\prime}<145^{\circ}$, the shock-associated noise mechanism generates a broadband spectra centered on a frequency $S t_{\text {BBSAN }}$ function of the angle. This frequency in the simulation agrees with the experimental data and with the Tam and Tanna's model. The azimuthal decomposition of the broadband shock-associated noise is dominated by the mode 1 in all upstream directions which is attributed to the excited mode 1 of the velocity in the shear layer. A particular shock-associated noise source radiating through a Mach wave-like mechanism is highlighted at large angles $\theta^{\prime}>145^{\circ}$. This noise component presents a bump corresponding to the screech frequency although the present jet is not screeching as expected for such a hot jet.

Finally, nonlinear effects are investigated. Both near and far field phenomena are highlighted. On the one hand, sharp pressure drops and raises are found in the vicinity of the shear layer which could correspond to the near field generation mechanism of crackle noise. On the other hand, the high acoustic levels clearly result in cumulative nonlinear effects in the far field, especially the peak direction, as suggested by the pressure derivative skewness or the wave steepening factor metrics. It justifies the use of a nonlinear acoustic solver.

\section{ACKNOWLEDGMENTS}

This study is supported by the French national space agency CNES and ONERA's scientific direction. The authors are grateful to H. Lambare as technical referee at CNES for launchers acoustic environment, E. Quémerais and G. Chaineray from ONERA for their efforts on CWIPI and CEDRE external coupling possibilities, and M. Huet from ONERA for his work on signal processing tools. Special thanks are extended to C. Peyret for his support and developments in the SPACE Euler code. 


\section{Appendix A: Parametric study for the geometrical tripping}

A short parametric study is conducted by varying the step main parameters, i.e. the axial location $x_{\text {step }}^{*}$, the height $h^{*}$ and the top edge slope as sketched in Figure 30. Five configurations have been investigated and the corresponding parameters are given in Table IV. The steps A, $\mathrm{B}$ and $\mathrm{C}$ are located in the convergent, the step D is located in the divergent and a configuration without step is used as reference. The nozzle geometry and the generating conditions are the same as for the full case. The free field domain downstream of the nozzle exit is however downsized and coarsened for computation cost reasons. The ambient pressure is slightly diminished compared to the nominal parameter $p_{\infty}$ to prevent any wall flow separation phenomenon which may occur for overexpanded exit conditions. The mesh characteristics in the nozzle are set to those provided in Table II for all configurations, except in the vicinity of the step when it exists, in order to quantify the tripping effects independently of the grid refinement. The vicinity of the step is discretized with tetrahedral elements connected to the hexahedral wall elements which explains the size drop at $x^{*}=x_{\text {step }}^{*}$ in Figure 2 and the important penalty in terms of cell number reported in Table IV.

The velocity fluctuations normalized by the jet exit velocity $\sqrt{\left(u_{x}^{\prime 2}+u_{r}^{\prime 2}+u_{\Theta}^{\prime 2}\right)} / u_{j}$ recorded along the wall and within the initial shear layer are drawn in Figure 31. The step height appears to have a major impact. The step A $\left(h^{*}=0.6 \%\right)$ provides similar fluctuation levels than the step-free configurations, i.e. less than $0.5 \%$ at the nozzle lips, while the steps $\mathrm{B}$ and $\mathrm{C}\left(h^{*}=1 \%\right)$ reach more than $3 \%$. The top edge slope modifies the wake flow but seems to have a limited influence on the levels inside the nozzle and slightly changes the growth rate downstream. The step D provides higher fluctuation levels than the reference, but this configuration illustrates that the trip cannot be placed in the divergent for a supersonic nozzle. The step strongly disturbs the whole flow because of the additional attached and reflected shocks downstream. It is chosen to implement the step $\mathrm{C}$ in the full computation since it leads to the highest fluctuation levels.

\section{REFERENCES}

${ }^{1}$ B. Chemoul, E. Louaas, P. Roux, D. Schmitt, and M. Pourcher, "Ariane 5 flight environments," Acta Astronautica 48, 275-285 (2001).

${ }^{2}$ C. Tam, "Supersonic jet noise," Annual Review of Fluid Mechanics 27, 17-43 (1995).

${ }^{3}$ C. Bailly and K. Fujii, "High-speed jet noise," Bulletin of the JSME 3, 1-13 (2016).

${ }^{4}$ C. Tam, K. Viswanathan, K. Ahuja, and J. Panda, "The source of jet noise: Experimental evidence," Journal of Fluid Mechanics 615, 253-292 (2008).

${ }^{5}$ B. Petitjean, K. Viswanathan, and D. McLaughlin, "Acoustic pressure waveforms measured in high speed jet noise experiencing nonlinear propagation," AIAA Journal 5, 193-215 (2006).

${ }^{6}$ J. Nichols, S. Lele, F. Ham, S. Martens, and J. Spyropoulos,
TABLE IV: step parameters in the parametric study.

\begin{tabular}{ccccccc}
\hline \hline step & zone & $x_{\text {step }}^{*}$ & $h^{*}$ & $w^{*}$ & slope & mesh size \\
\hline none & - & - & 0.0 & - & - & $29.4 \times 10^{6}$ \\
A & convergent & -1.38 & 0.006 & 0.007 & $/ /$ wall & $37.9 \times 10^{6}$ \\
B & convergent & -1.38 & 0.010 & 0.007 & $/ /$ wall & $40.7 \times 10^{6}$ \\
C & convergent & -1.38 & 0.010 & 0.007 & $/ / \vec{x}$ & $42.2 \times 10^{6}$ \\
D & divergent & -1.08 & 0.006 & 0.006 & $/ / \vec{x}$ & $47.9 \times 10^{6}$ \\
\hline \hline
\end{tabular}

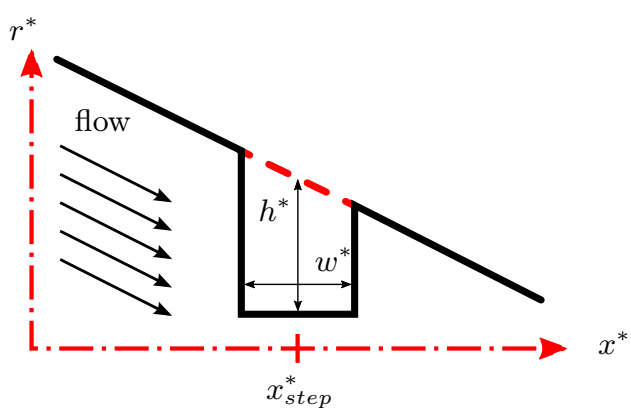

(a)

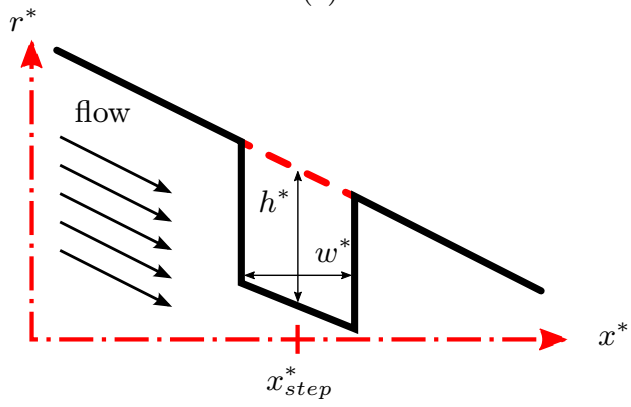

(b)

FIG. 30: nozzle step configurations with a step edge slope (Va) parallel to $\vec{x}$ or (Vb) parallel to the wall.

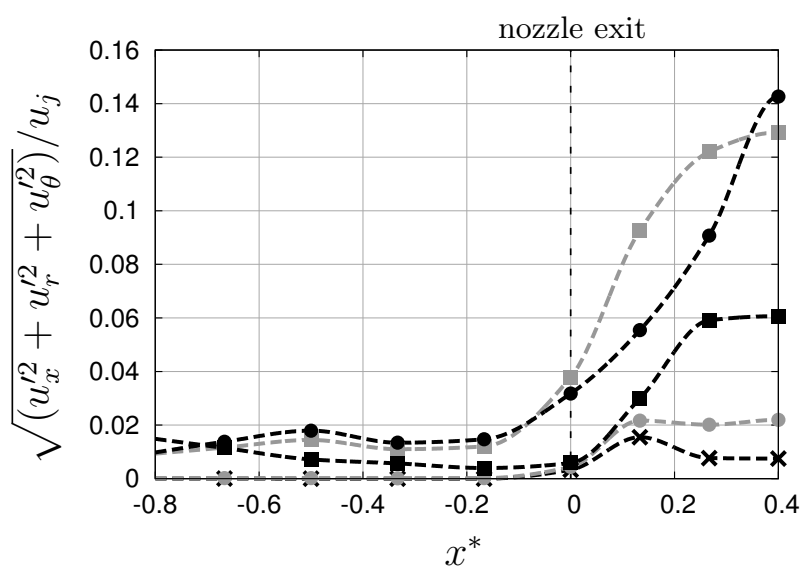

FIG. 31: fluctuating velocity intensity in the nozzle boundary layer and the free jet initial shear layer. --X-- : without step; --O-- : step A; --1-- : step B; --०-- : step C; - -n- - : step D. 
"Crackle noise in heated supersonic jets," Journal of Engineering for Gas Turbines and Power 135, 1-7 (2013).

${ }^{7}$ W. Baars, C. Tinney, M. Wochner, and M. Hamilton, "On cumulative nonlinear acoustic wave distortions from high-speed jets," Journal of Fluid Mechanics 749, 331-366 (2014).

${ }^{8}$ P. Mora, J. Kastner, and E. Gutmark, "Impact of heat on the pressure skewness and kurtosis in supersonic jets," AIAA Journal 52, 777-787 (2014).

${ }^{9}$ J. Panda, "Shock oscillation in underexpanded screeching jets," Journal of Fluid Mechanics 363, 173-198 (1998).

${ }^{10}$ A. Krothapalli, E. Rajkuperan, F. Alvi, and L. Lourenco, "Flow field and noise characteristics of a supersonic impinging jet," Journal of Fluid Mechanics 392, 155-181 (1999).

${ }^{11}$ D. Edgington-Mitchell, K. Oberleithner, D. Honnery, and J. Soria, "Coherent structure and sound production in the helical mode of a screeching axisymmetric jet," Journal of Fluid Mechanics 748, 822-847 (2014).

${ }^{12}$ T. Ishii, S. Tsutsumi, K. Ui, S. Tokudome, Y. Ishii, K. Wada, and S. Nakamura, "Acoustic measurement of 1 : 42 scale booster and launch pad," in $164^{\text {th }}$ Meeting of the Acoustical Society of America, Vol. 18 (Kansas City, MO, USA, 2012) pp. 1-13.

${ }^{13}$ D. Munday, N. Heeb, and E. Gutmark, "Acoustic effects of chevrons on supersonic jets exiting conical convergent-divergent nozzles," AIAA Journal 50, 2336-2350 (2012).

${ }^{14}$ W. Horne, N. Burnside, J. Panda, and C. Brodell, "Measurements of unsteady pressure fluctuations in the near-field of a solid rocket motor plume," International Journal of Aeroacoustics 15, 554-569 (2016).

${ }^{15}$ J. Varnier, V. Koudriavstsev, and A. Safronov, "Simplified approach of jet aerodynamics with a view to acoustics," AIAA Journal 44, 1690-1693 (2006).

${ }^{16}$ T. Nonomura and K. Fujii, "Overexpansion effects on characteristics of Mach waves from a supersonic cold jet," AIAA Journal 49, 2282-2294 (2011).

${ }^{17}$ R. Gojon, C. Bogey, and O. Marsden, "Investigation of tone generation in ideally expanded supersonic planar impinging jets using large-eddy simulation," Journal of Fluid Mechanics 808, 90-115 (2016).

${ }^{18} \mathrm{C}$. Bogey and R. Gojon, "Feedback loop and upwind-propagating waves in ideally expanded supersonic impinging round jets," Journal of Fluid Mechanics 823, 562-591 (2017).

${ }^{19}$ N. de Cacqueray and C. Bogey, "Noise of an overexpanded Mach 3.3 jet: Non-linear propagation effects and correlations with flows," International Journal of Aeroacoustics 13, 607-632 (2014).

${ }^{20}$ G. Brès, F. Ham, J. Nichols, and S. Lele, "Unstructured largeeddy simulations of supersonic jets," AIAA Journal 55, 11641184 (2017).

${ }^{21}$ C. Bogey, O. Marsden, and C. Bailly, "Influence of initial turbulence level on the flow and sound fields of a subsonic jet at a diameter-based Reynolds number of $10^{5}$," Journal of Fluid Mechanics 701, 352-385 (2012).

${ }^{22}$ J. Liu, K. Kailasanath, J. Boris, N. Heeb, D. Munday, and E. Gutmark, "Effect of the initial turbulence level on an underexpanded supersonic jet," AIAA Journal 51, 741-744 (2013).

${ }^{23}$ M. Lorteau, F. Cléro, and F. Vuillot, "Analysis of noise radiation mechanism in hot subsonic jet from a validated large eddy simulation solution," Physics of Fluids 27, 1-29 (2015).

${ }^{24}$ G. Brès, P. Jordan, V. Jaunet, M. Le Rallic, A. Cavalieri, A. Towne, S. Lele, T. Colonius, and O. Schmidr, "Importance of the nozzle-exit boundary-layer state in subsonic turbulent jets," Journal of Fluid Mechanics 851, 83-124 (2018).

${ }^{25}$ D. Long, T. McDonald, and P. Maye, "Effect of inlet flow conditions on noise and performance of supersonic nozzles," in $16^{\text {th }}$ AIAA/CEAS Aeroacoustics Conference, 2010-3920 (Stockholm, Sweden, 2010).

${ }^{26}$ A. Hussain and M. Zedan, "Effects of the initial condition on the axisymmetric free shear layer: Effect of the initial fluctuation level," Physics of Fluids 21, 1475-1481 (1978).

${ }^{27}$ R. Fontaine, G. Elliott, J. Austin, and J. Freund, "Very near- nozzle shear-layer turbulence and jet noise," Journal of Fluid Mechanics 770, 27-51 (2015).

${ }^{28} \mathrm{C}$. Bogey and O. Marsden, "Identification of the effects of the nozzle-exit boundary-layer thickness and its corresponding Reynolds number in initially highly disturbed subsonic jets," Physics of Fluids 25, 1-27 (2013).

${ }^{29}$ M. Gloor, S. Buhler, and L. Kleiser, "Transition to turbulence and noise radiation in heated coaxial jet flows," Physics of Fluids 28, 1-28 (2016).

${ }^{30} \mathrm{C}$. Bogey, O. Marsden, and C. Bailly, "Large-eddy simulation of the flow and acoustic field of a Reynolds number $10^{5}$ subsonic jet with tripped exit boundary layers," Physics of Fluids 23, 1-20 (2011).

${ }^{31}$ G. Rahier, J. Prieur, F. Vuillot, N. Lupoglazoff, and A. Biancherin, "Investigation of integral surface formulations for acoustic post-processing of unsteady aerodynamic jet simulations," Aerospace Science and Technology 8, 453-467 (2004).

${ }^{32}$ G. Djambazov, C.-H. Lai, and K. Pericleous, "On the coupling of Navier-Stokes and linearied Euler equations for aeroacoustic simulation," Computing and Visualization in Science 3, 9-12 (2000).

${ }^{33}$ C. Bogey, S. Barré, D. Juvé, and C. Bailly, "Simulation of a hot coaxial jet: Direct noise prediction and flow-acoustics correlations," Physics of Fluids 21, 1-14 (2009).

${ }^{34}$ N. de Cacqueray, C. Bogey, and C. Bailly, "Investigation of a high-Mach-number overexpanded jet using large-eddy simulation," AIAA Journal 49, 2171-2182 (2011).

${ }^{35}$ R. Harris, E. Collins, E. Luke, A. Sescu, L. Strutzenberg, and J. West, "Hybrid discontinuous Galerkin and finite volume method for launch environment acoustics prediction," AIAA Journal 53, 3430-3447 (2015).

${ }^{36}$ R. Harris, E. Collins, E. Luke, and A. Sescu, "Coupled overset unstructured discontinuous Galerkin method for launch environment acoustics prediction," AIAA Journal 54, 1932-1952 (2016).

${ }^{37}$ O. Labbé, C. Peyret, G. Rahier, and M. Huet, "A CFD/CAA coupling method applied to jet noise prediction," Computers \& Fluids 86, 1-13 (2013).

${ }^{38}$ S. Tsutsumi, S. Ishii, K. Ui, S. Tokudome, and K. Wada, "Assessing prediction and reduction technique of lift-off acoustics using epsilon flight data," in 53 $3^{\text {rd }}$ AIAA Aerospace Sciences Meeting, 2015-1007 (Kissimmee, FL, USA, 2015).

${ }^{39}$ A. Langenais, F. Vuillot, C. Peyret, G. Chaineray, and C. Bailly, "Assessment of a two-way coupling methodology between a flow and a high-order nonlinear acoustic unstructured solvers," Flow, Turbulence and Combustion (2018), 10.1007/s10494-018-9928-0.

${ }^{40}$ A. Krothapalli, L. Venkatakrishnan, and L. Lourenco, "Crackle: A dominant component of supersonic jet mixing noise," in $6^{\text {th }}$ AIAA/CEAS Aeroacoustics Conference, 2000-2024 (Lahaina, HI, USA, 2000).

${ }^{41}$ M. Shepherd, K. Gee, and M. Wochner, "Short-range shock formation and coalescence in numerical simulation of broadband noise propagation," Journal of the Acoustical Society of America 126, 2886-2893 (2009).

${ }^{42}$ M. Muhlestein, K. Gee, T. Neilsen, and D. Thomas, "Prediction of nonlinear propagation of noise from a solid rocket motor," in $164^{\text {th }}$ Meeting of the Acoustical Society of America, Vol. 18 (2012).

${ }^{43}$ B. Reichman, M. Muhlestein, K. Gee, T. Neilsen, and D. Thomas, "Evolution of the derivative skewness for nonlinearly propagating waves," Journal of the Acoustical Society of America 139, 1390-1403 (2016).

${ }^{44}$ A. Langenais, F. Vuillot, J. Troyes, and C. Bailly, "Numerical investigation of the noise generated by a rocket engine at liftoff conditions using a two-way coupled CFD-CAA method." in $23^{\text {rd }}$ AIAA/CEAS Aeroacoustics Conference, 2017-3212 (Denver, CO, USA, 2017).

${ }^{45}$ J. Troyes, F. Vuillot, H. Lambaré, and A. Espinosa Ramos, "Numerical study of free supersonic hot jet on unstructured grids with emphasis on aerodynamics and resulting radiated noise," in 22 $2^{\text {nd }}$ AIAA/CEAS Aeroacoustics Conference, 2016-2734 (Lyon, France, 2016). 
${ }^{46}$ C. Hirsch, Numerical Computation of Internal and External Flows, Volume 2: Computational Methods for Inviscid and Viscous Flows, 978-0471924524 (John Wiley \& Sons, 1990).

${ }^{47}$ A. Refloch, B. Courbet, A. Murrone, P. Villedieu, C. Laurent, P. Gilbank, J. Troyes, L. Tessé, G. Chaineray, J.-B. Dargaud, E. Quémerais, and F. Vuillot, "CEDRE software," Aerospace Lab Journal 2, 1-10 (2011).

${ }^{48}$ P. Delorme, P. Mazet, C. Peyret, and Y. Ventribout, "Computational aeroacoustics applications based on a discontinuous Galerkin method," Comptes Rendus Mécanique 333, 676-682 (2005).

${ }^{49}$ R. Léger, C. Peyret, and S. Piperno, "Coupled discontinuous Galerkin / finite difference solver on hybrid meshes for computational aeroacoustics," AIAA Journal 50, 338-349 (2012).

${ }^{50}$ C. Tam, "Computational aeroacoustics: Issues and methods," AIAA Journal 33, 1788-1796 (1995).

51"ISO 9613-1:1993 - acoustics - attenuation of sound during propagation outdoors - part 1: Air absorption," (1993).

${ }^{52}$ E. Quémerais, "Coupling with interpolation parallel interface," ONERA web site (2016).

${ }^{53}$ J. Liu, A. Corrigan, K. Kailasanath, R. Ramamurti, and E. Gutmark, "Helical screech tone generation in overexpanded jets," in 23 $3^{\text {rd }}$ AIAA/CEAS Aeroacoustics Conference, 2017-3208 (Denver, CO, USA, 2017).

${ }^{54}$ R. Narasimha and K. Sreenivasan, "Relaminarization of fluid flows," Advances in Applied Mechanics 19, 221-309 (1979).

${ }^{55}$ E. Morrisette and T. Goldberg, "Turbulent-flow separation criteria for overexpanded supersonic nozzles," Technical Paper 1207 (NASA, Hampton, VA, USA, 1978).

${ }^{56}$ H. Schlichting and K. Gersyen, Boundary-Layer Theory, 7th ed., 978-3662529171 (Springer, 1979).

${ }^{57}$ T. Maeder, N. Adams, and L. Kleiser, "Direct simulation of turbulent supersonic boundary layers by an extended temporal approach," Journal of Fluid Mechanics 429, 187-216 (2001).

${ }^{58}$ L. Jacquin, M. S., P. Geffroy, and F. Cruand, "Mixing of a heated supersonic jet with a supersonic parallel stream," in $5^{\text {th }}$ European Turbulence Conference (Siena, Italy, 1994).

${ }^{59} \mathrm{C}$. Tam and H. Tanna, "Shock-associated noise of supersonic jets from convergent-divergent nozzles," Journal of Sound and Vibration 81, 337-358 (1982).

${ }^{60}$ C. Tam, S. Parrish, and K. Viswanathan, "Harmonics of jet screech tones," AIAA Journal 52, 2471-2479 (2014).

${ }^{61} \mathrm{~T}$. Troutt and D. McLaughlin, "Experiments on the flow and acoustic properties of a moderate-Reynolds-number supersonic jet," Journal of Fluid Mechanics 116, 123-156 (1982).

${ }^{62}$ B. Greska, A. Krothapalli, W. Horne, and N. Burnside, "A near-field study of high temperature supersonic jets," in $14^{\text {th }}$ AIAA/CEAS Aeroacoustics Conference, 2008-3026 (Vancouver, Canada, 2008).

${ }^{63}$ S. Lele and J. Larsson, "Shock-turbulence interaction: What we know and what we can learn from peta-scale simulations," Journal of Physics: Conference Series 180, 1-10 (2009).

${ }^{64}$ K. Gee, V. Sparrow, M. James, J. Downing, C. Hobbs, T. Gabrielson, and A. Atchley, "The role of nonlinear effects in the propagation of noise from high-power jet aircraft," Journal of the Acoustical Society of America 123, 4082-4093 (2008).

${ }^{65}$ A. Vaughn, T. Neilsen, K. Gee, A. Wall, J. Downing, and M. James, "Broadband shock-associated noise from a highperformance military aircraft," Journal of the Acoustical Society of America 144, 242-247 (2018).

${ }^{66}$ H. Oertel, F. Seiler, J. Srulijes, and R. Hruschka, "Mach waves produced in the supersonic jet mixing layer by shock/vortex interaction," Shock Waves 26, 231-240 (2016).

${ }^{67}$ J. Panda and R. Seasholtz, "Experimental investigation of density fluctuations in high-speed jets and correlation with generated noise," Journal of Fluid Mechanics 450, 97-130 (2002).

${ }^{68} \mathrm{C}$. Bogey and C. Bailly, "An analysis of the correlations between the turbulent flow and the sound pressure field of subsonic jets," Journal of Fluid Mechanics 583, 71-97 (2007).

${ }^{69}$ R. Schlinker, S. Liljenberg, D. Polak, K. Post, C. Chipman, and
A. Stern, "Supersonic jet noise source characteristics \& propagation: Engine and model scale," in $13^{\text {th }}$ AIAA/CEAS Aeroacoustics Conference, 2007-3623 (Rome, Italy, 2007).

${ }^{70} \mathrm{~S}$. McInerny, "Launch vehicle acoustics part II: Statistics of the time domain data," Journal of Aircraft 33, 518-523 (1996).

${ }^{71} \mathrm{~W}$. Baars and C. Tinney, "Shock-structures in the acoustic field of a Mach 3 jet with crackle," Journal of Sound and Vibration 333, 2539-2553 (2014).

${ }^{72} \mathrm{P}$. Pineau and C. Bogey, "Numerical study of the sound fiels of temporally-developing supersonic round jets," in $23^{\text {rd }}$ AIAA/CEAS Aeroacoustics Conference, 2017-3209 (Denver, CO, USA, 2017).

${ }^{73}$ M. Muhlestein, K. L. Gee, T. Neilsen, and D. Thomas, "Evolution of the average steepening factor for nonlinearly propagating waves," Journal of the Acoustical Society of America 137, 640650 (2015). 